\title{
Stereoselective assembly of multifunctional spirocyclohexene pyrazolones that induce autophagy-dependent apoptosis in colorectal cancer cells
}

Xiang Li,${ }^{\dagger}$ Fei-Yu Chen,${ }^{\dagger}$ Jing-Wen Kang, ${ }^{\dagger}$ Jin Zhou, ${ }^{\dagger}$ Cheng Peng, ${ }^{* \dagger}$ Wei Huang, ${ }^{\dagger}$ Mu-Ke Zhou, ${ }^{\ddagger}$ Gu He ${ }^{* \sharp}$ and Bo Han*i

† State Key Laboratory of Southwestern Chinese Medicine Resources, School of Pharmacy, Chengdu University of Traditional Chinese Medicine, Chengdu 611137, China.

E-mail:pengcheng@cdutcm.edu.cn or hanbo@cdutcm.edu.cn

$\$$ State Key Laboratory of Biotherapy and Department of Dermatology, West China Hospital, Sichuan University and Collaborative Innovation Center for Biotherapy, Chengdu, 610041, China.

E-mail: hegu@scu.edu.cn

\section{Supporting Information}

Table of Contents

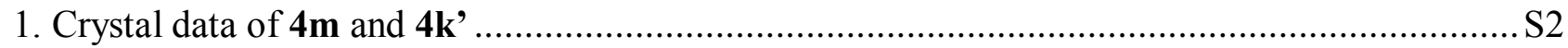

2. NMR spectra and HPLC chromatograms .................................................................. S6

3. The anti-proliferative IC50 values against a panel of cancer cell lines (Table S1) ..................S88 


\section{Crystal data of $4 \mathrm{~m}$ and $4 \mathrm{k}^{\prime}$}

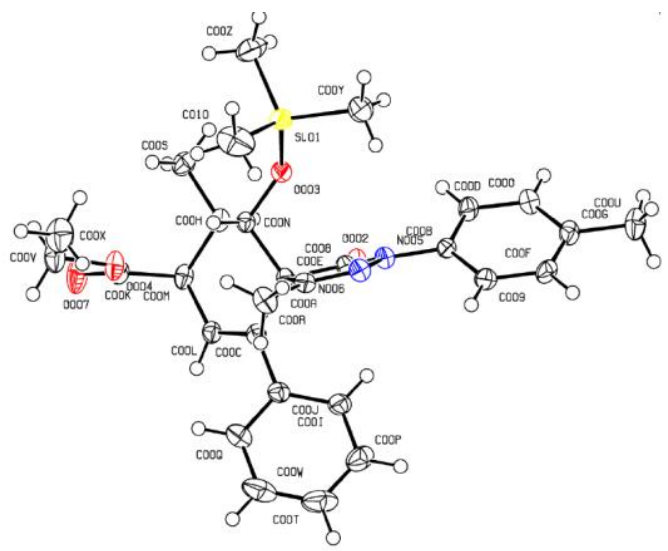

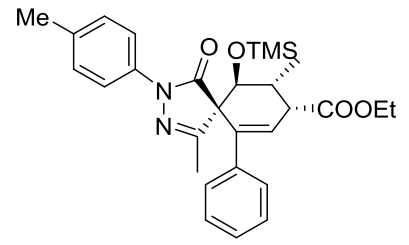

4m CCDC 1886929

Figure S1 X-ray crystal structure of $\mathbf{4 m}$. The thermal ellipsoids are drawn at a $50 \%$ probability level.

Single crystals suitable for XRD were obtained by vapor diffusion experiment: compound $\mathbf{4 m}$ $(5 S, 8 R, 9 R, 10 S, 50 \mathrm{mg}$,) was dissolved in $0.5 \mathrm{~mL}$ dichloromethane and $1.0 \mathrm{~mL}$ methanol in a glass vial, which was then placed in sealed glass container. Crystals were obtained in about 4-5 days.

\begin{tabular}{|c|c|}
\hline Identification code & lq-18012702-139k \\
\hline Empirical formula & $\mathrm{C}_{29} \mathrm{H}_{36} \mathrm{~N}_{2} \mathrm{O}_{4} \mathrm{Si}$ \\
\hline Formula weight & 504.69 \\
\hline Temperature/K & 139.1(2) \\
\hline Crystal system & orthorhombic \\
\hline Space group & $\mathrm{P} 212121$ \\
\hline $\mathrm{a} / \AA$ & $8.96693(9)$ \\
\hline $\mathrm{b} / \AA$ & $16.8380(2)$ \\
\hline $\mathrm{c} / \AA$ & $18.4036(3)$ \\
\hline$\alpha /^{\circ}$ & 90 \\
\hline$\beta /{ }^{\circ}$ & 90 \\
\hline$\gamma /{ }^{\circ}$ & 90 \\
\hline Volume/Å ${ }^{3}$ & $2778.68(6)$ \\
\hline $\mathrm{Z}$ & 4 \\
\hline$\rho_{\text {calc }} \mathrm{g} / \mathrm{cm}^{3}$ & 1.206 \\
\hline$\mu / \mathrm{mm}^{-1}$ & 1.031 \\
\hline $\mathrm{F}(000)$ & 1080.0 \\
\hline Crystal size $/ \mathrm{mm}^{3}$ & $0.6 \times 0.4 \times 0.25$ \\
\hline Radiation & $\mathrm{CuK} \alpha(\lambda=1.54184)$ \\
\hline $2 \Theta$ range for data collection $/{ }^{\circ}$ & 7.116 to 145.916 \\
\hline Index ranges & $-8 \leq \mathrm{h} \leq 11,-19 \leq \mathrm{k} \leq 20,-21 \leq 1 \leq 22$ \\
\hline Reflections collected & 12506 \\
\hline Independent reflections & $5418\left[\mathrm{R}_{\text {int }}=0.0368, \mathrm{R}_{\text {sigma }}=0.0471\right]$ \\
\hline Data/restraints/parameters & $5418 / 0 / 332$ \\
\hline
\end{tabular}


Goodness-of-fit on $\mathrm{F}^{2}$

Final $\mathrm{R}$ indexes $[\mathrm{I}>=2 \sigma(\mathrm{I})]$

Final R indexes [all data]

Largest diff. peak/hole / e $\AA^{-3}$

Flack parameter
1.054

$\mathrm{R}_{1}=0.0552, \mathrm{wR}_{2}=0.1337$

$\mathrm{R}_{1}=0.0582, \mathrm{wR}_{2}=0.1371$

0.43/-0.42

$-0.007(19)$ 


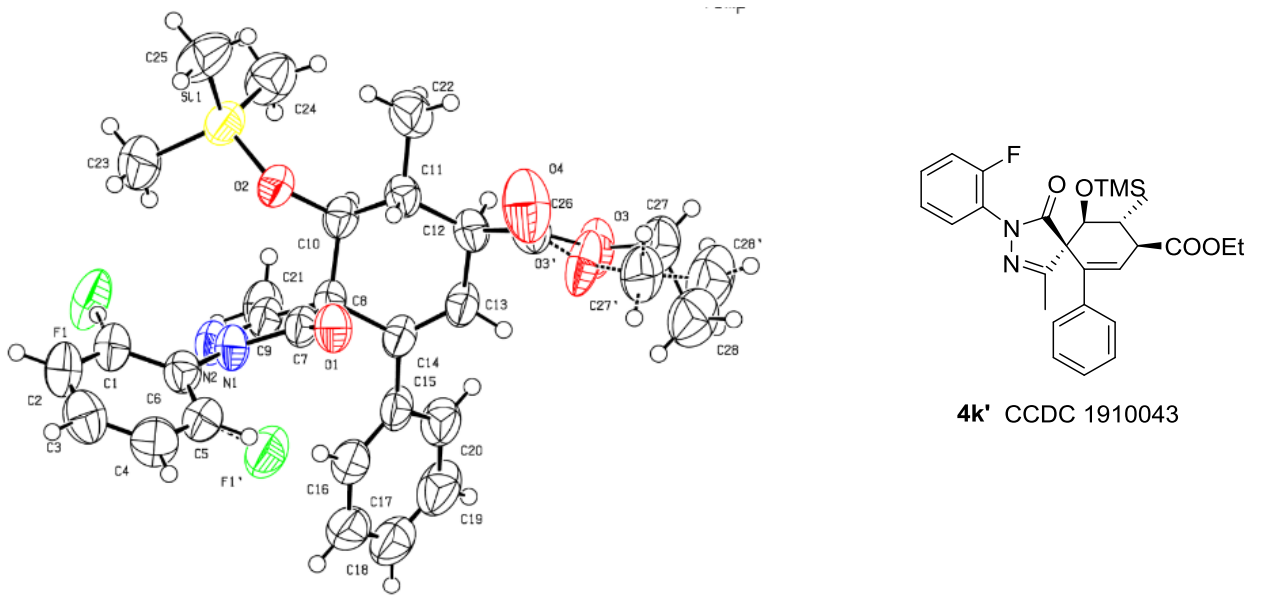

Figure S2 X-ray crystal structure of $\mathbf{4} \mathbf{k}^{\prime}$. The thermal ellipsoids are drawn at a 50\% probability level.

Single crystals suitable for XRD were obtained by vapor diffusion experiment: compound 4k' (50 $\mathrm{mg}$ ) was dissolved in $2.0 \mathrm{~mL}$ methanol in a glass vial, which was then placed in sealed glass container. Crystals were obtained in about 5-6 days.

$\begin{array}{ll}\text { Identification code } & \text { exp_10192 } \\ \text { Empirical formula } & \mathrm{C}_{28} \mathrm{H}_{33} \mathrm{FN}_{2} \mathrm{O}_{4} \mathrm{Si} \\ \text { Formula weight } & 508.65 \\ \text { Temperature/K } & 293(2) \\ \text { Crystal system } & \mathrm{N} / \mathrm{A} \\ \text { Space group } & \mathrm{P}_{1} / \mathrm{c} \\ \mathrm{a} / \AA & 18.8993(18) \\ \mathrm{b} / \AA & 14.9288(11) \\ \mathrm{c} / \AA & 9.7511(8) \\ \alpha /{ }^{\circ} & 90.00 \\ \beta /{ }^{\circ} & 97.082(9) \\ \gamma /{ }^{\circ} & 90.00 \\ \text { Volume/ } \AA^{3} & 2730.2(4) \\ \mathrm{Z} & 4 \\ \rho_{\text {calc }} \mathrm{g} / \mathrm{cm}^{3} & 1.237 \\ \mu / \mathrm{mm}^{-1} & 1.109 \\ \mathrm{~F}(000) & 1080.0 \\ \text { Crystal size/mm } & \\ \text { Radiation } & 0.08 \times 0.08 \times 0.07 \\ 2 \Theta \text { range for data collection/ } & \mathrm{CuK} \alpha(\lambda=1.54184) \\ \text { Index ranges } & 7.56 \text { to } 134.5 \\ \text { Reflections collected } & -22 \leq \mathrm{h} \leq 18,-13 \leq \mathrm{k} \leq 17,-9 \leq 1 \leq 11 \\ \text { Independent reflections } & 9790 \\ \text { Data/restraints/parameters } & 4891\left[\mathrm{R}_{\text {int }}=0.0609, \mathrm{R}_{\text {sigma }}=\mathrm{N} / \mathrm{A}\right] \\ \text { Goodness-of-fit on } \mathrm{F}^{2} & 4891 / 2 / 331 \\ & 1.215 \\ & \end{array}$


Final $\mathrm{R}$ indexes $[\mathrm{I}>=2 \sigma(\mathrm{I})]$

Final $\mathrm{R}$ indexes [all data]

Largest diff. peak/hole / e $\AA^{-3}$
$\mathrm{R}_{1}=0.0860, \mathrm{wR}_{2}=0.1880$

$\mathrm{R}_{1}=0.1485, \mathrm{wR}_{2}=0.2256$

$1.10 /-0.32$ 


\section{NMR spectra and HPLC chromatograms}
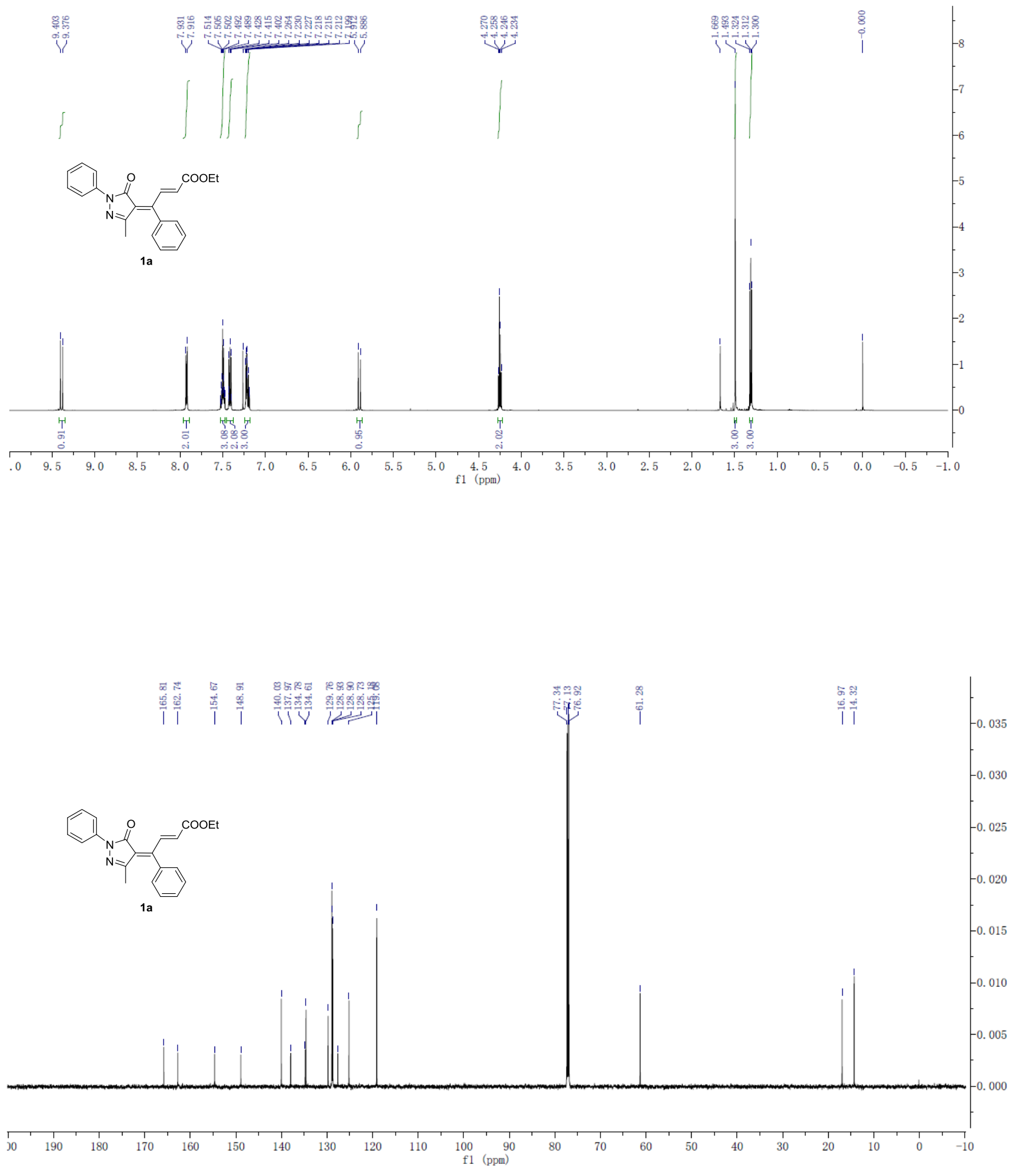

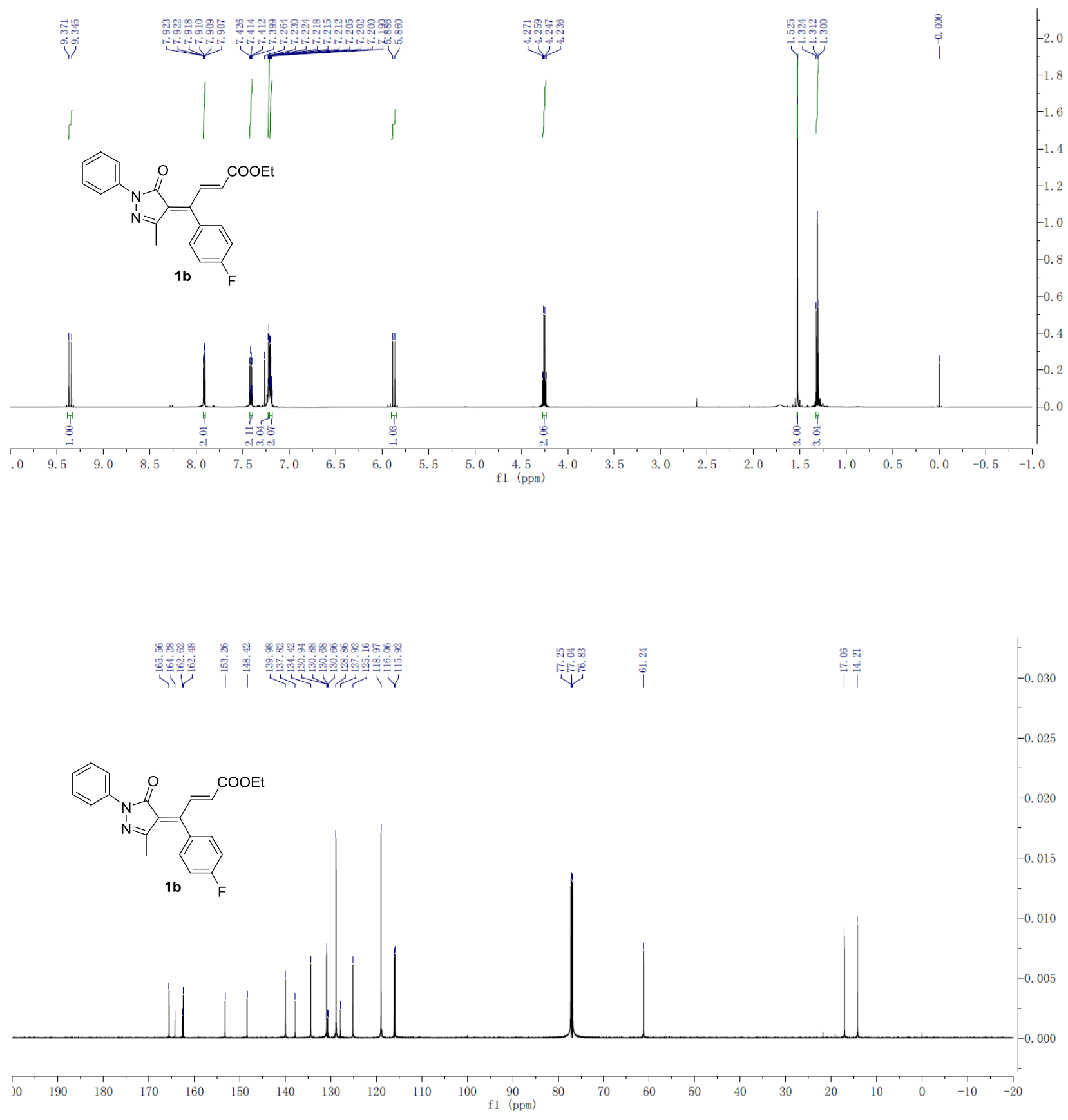

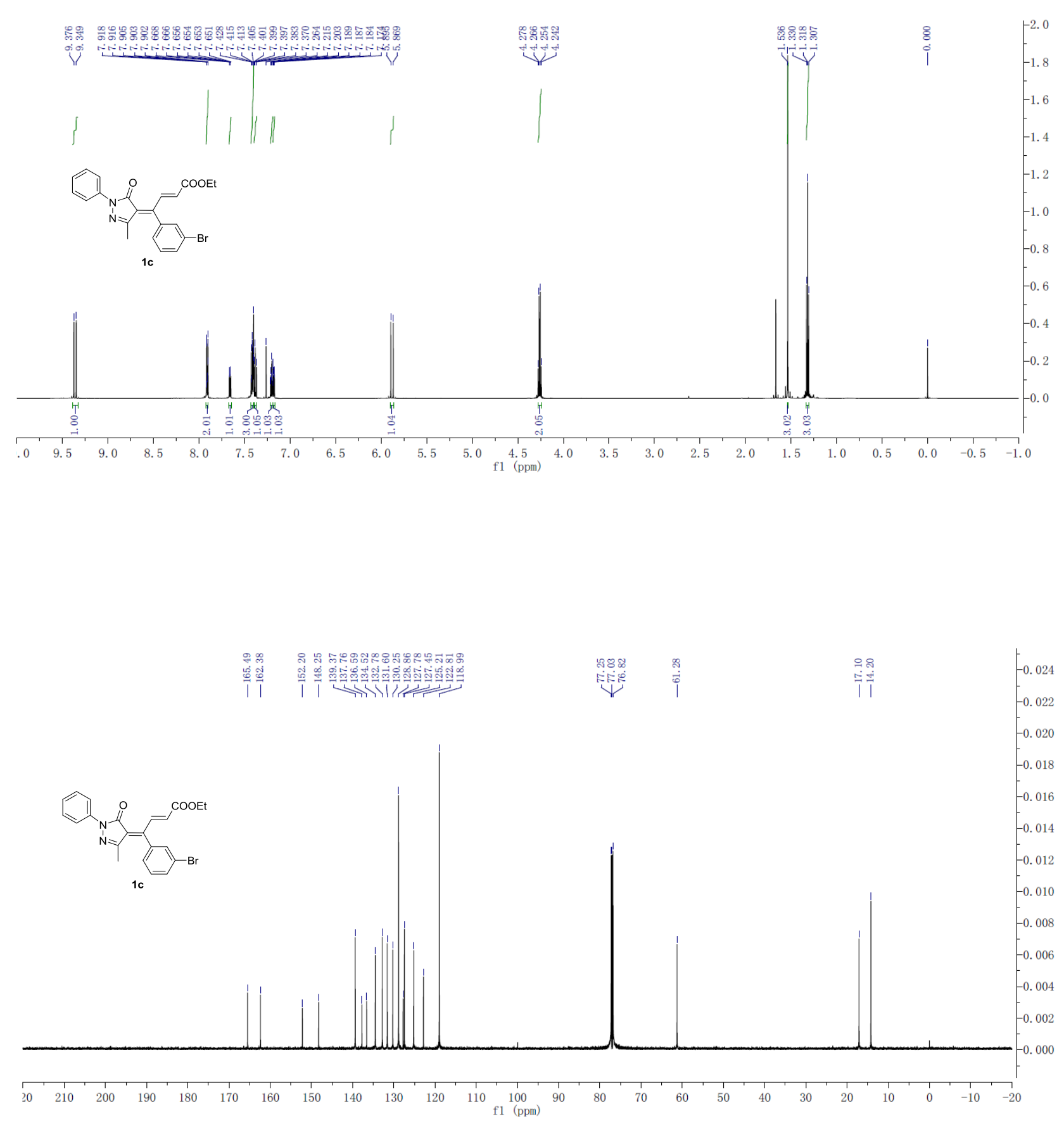

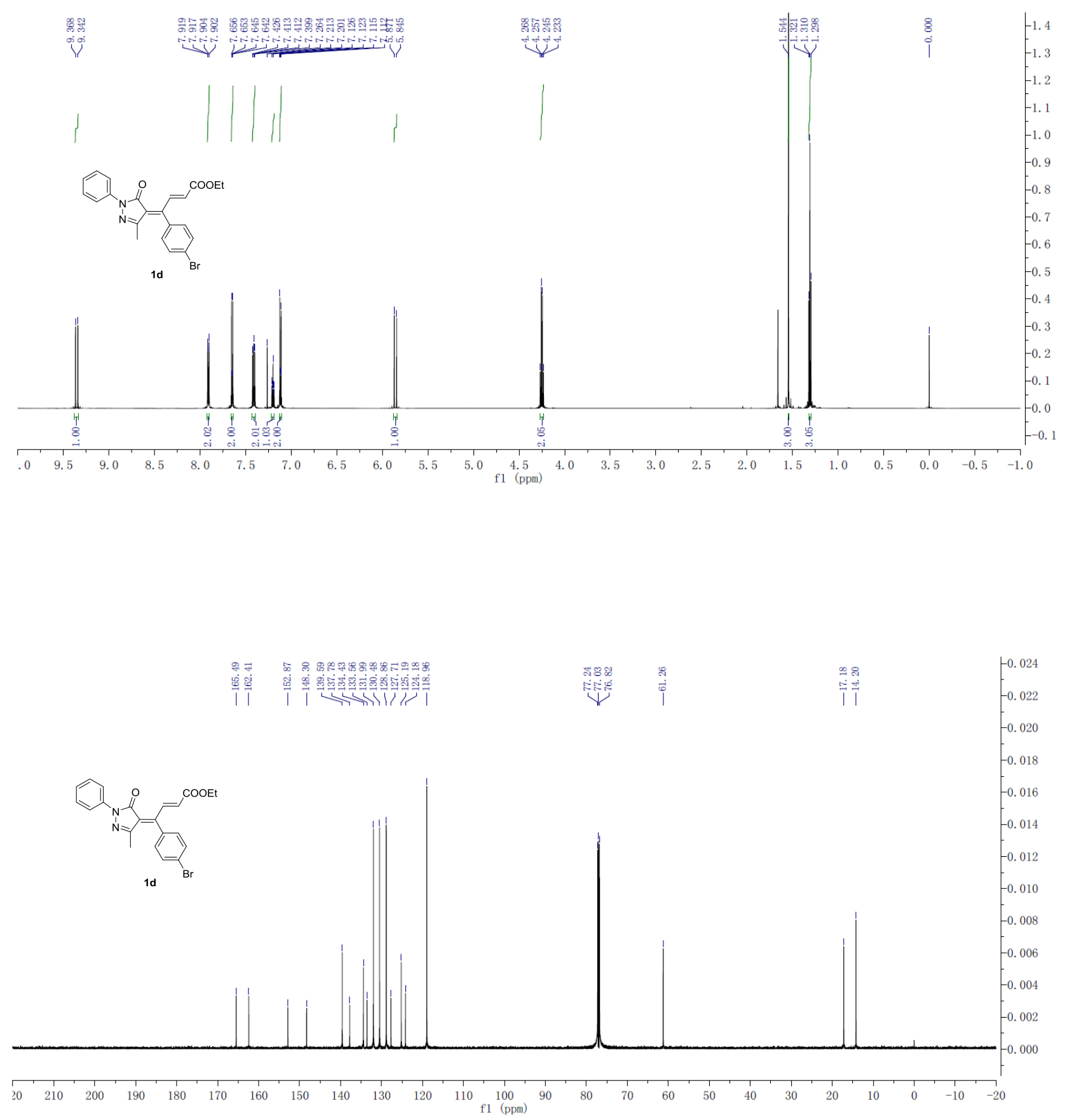

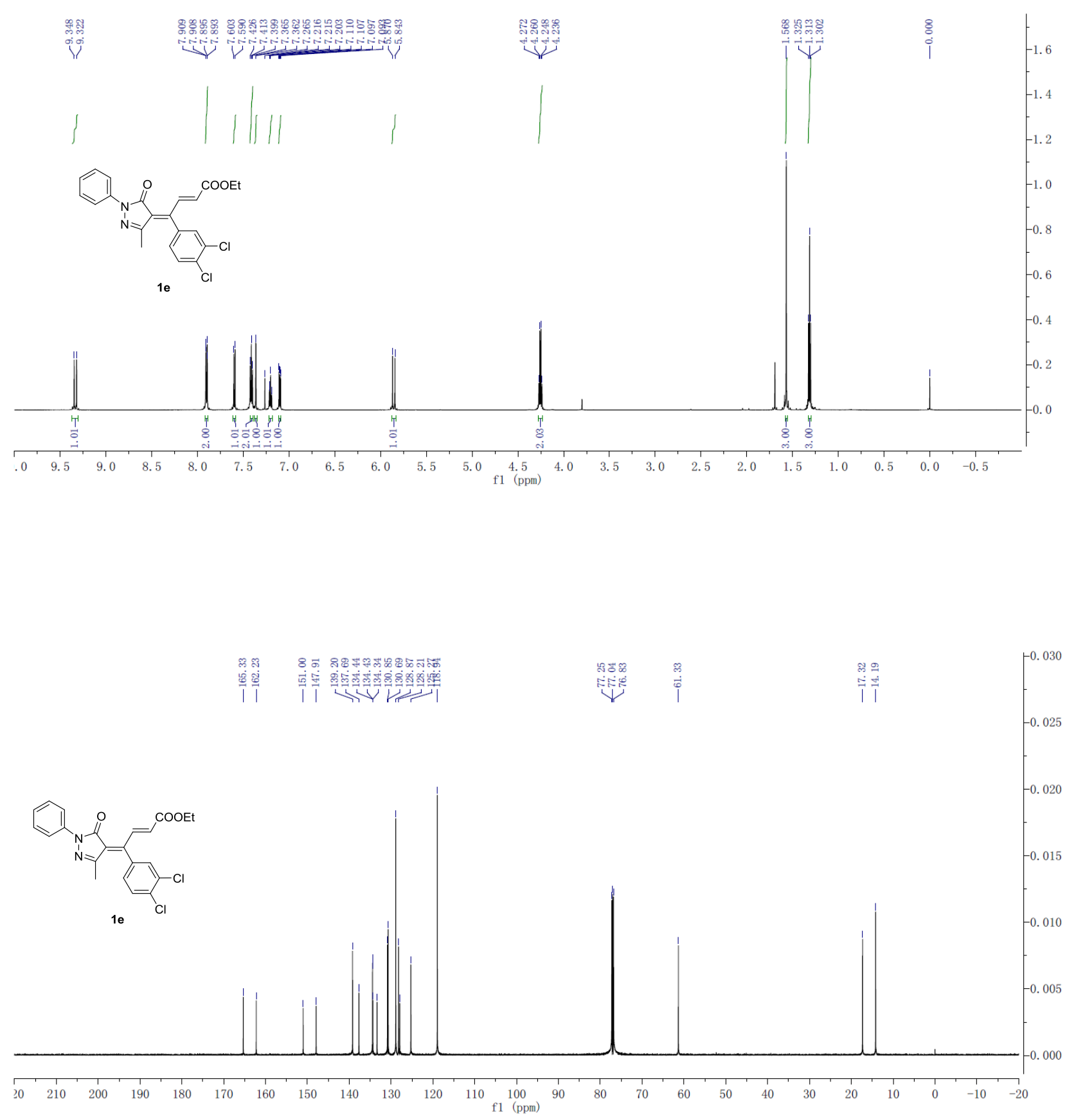

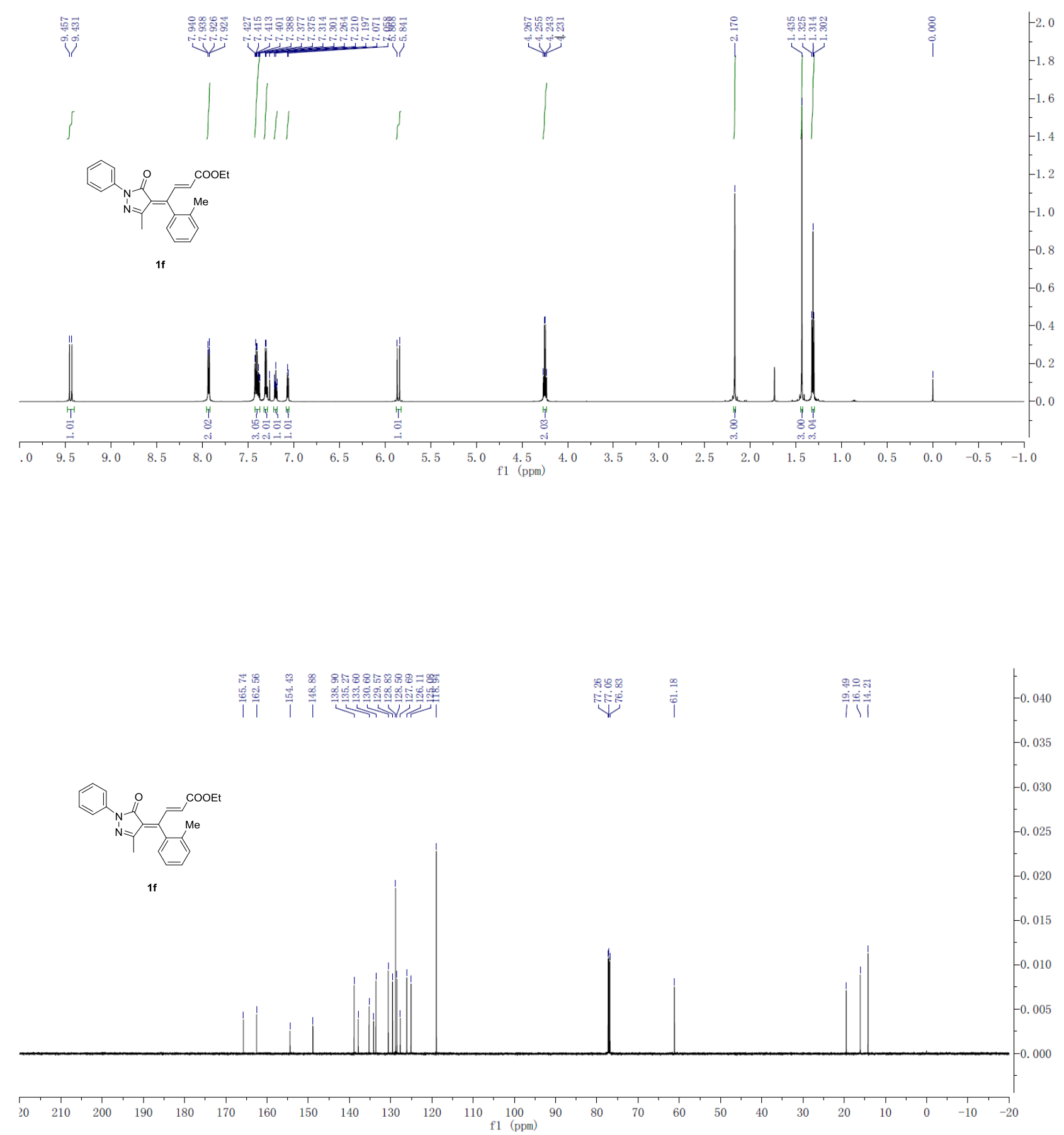

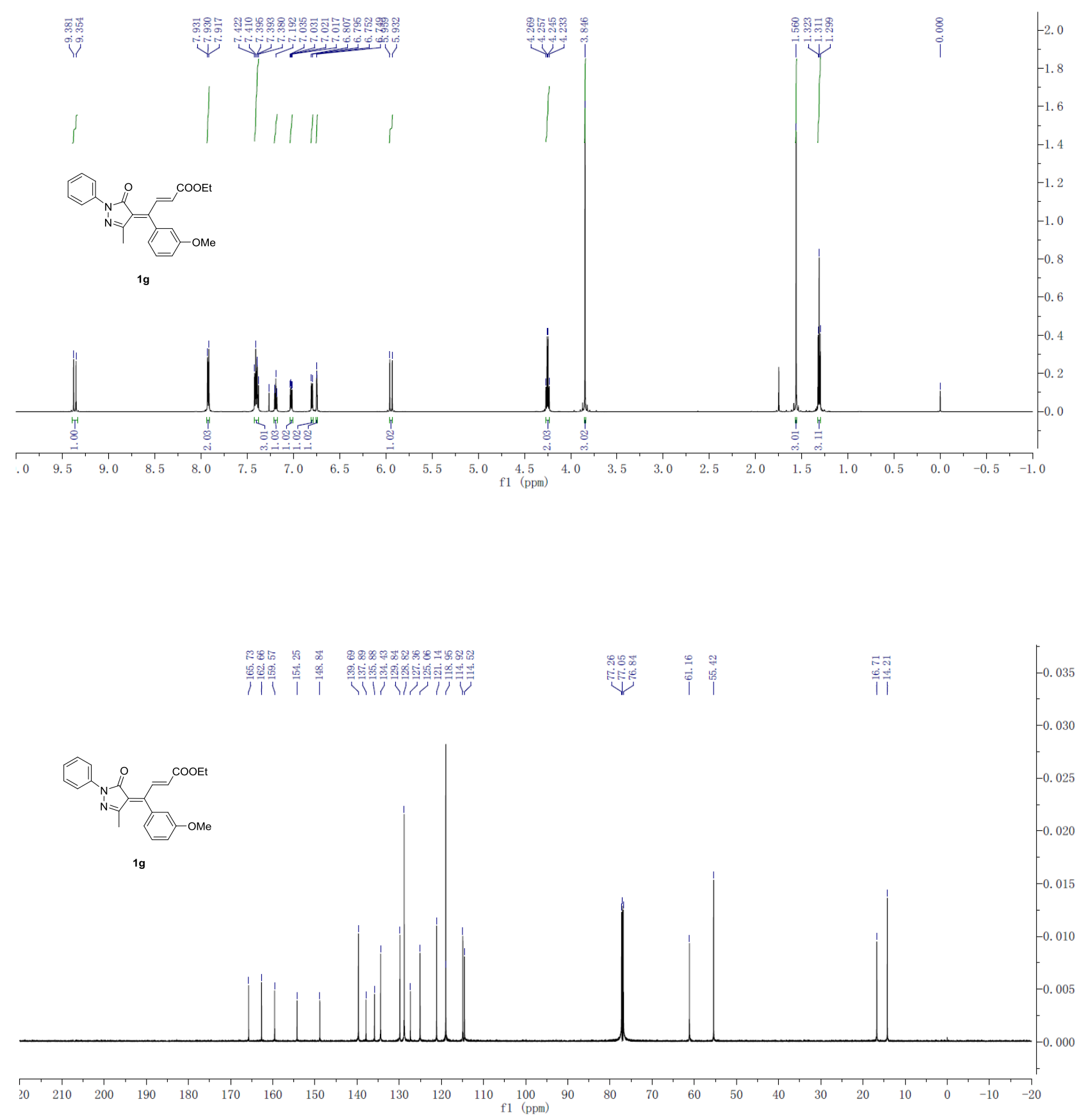


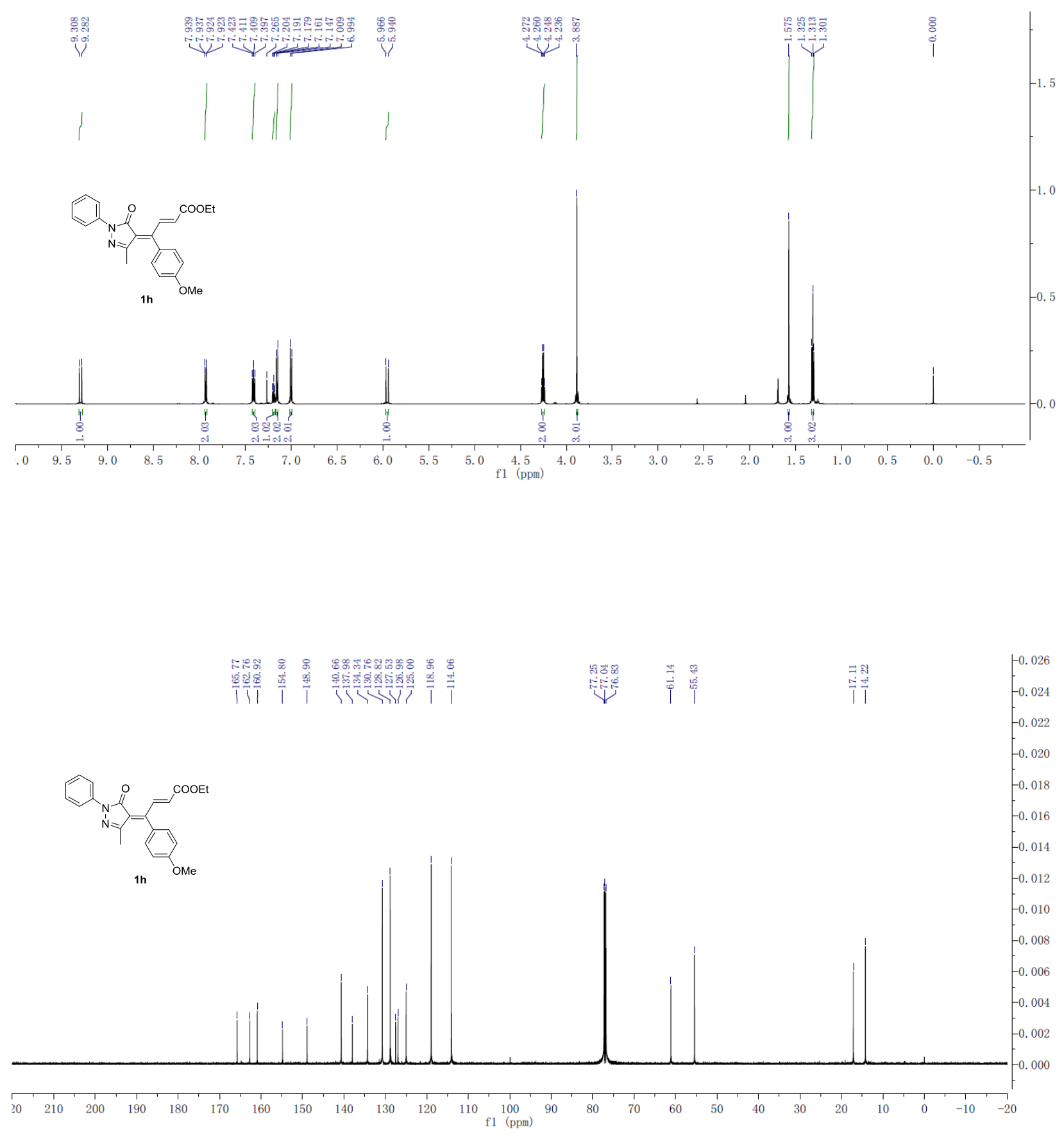



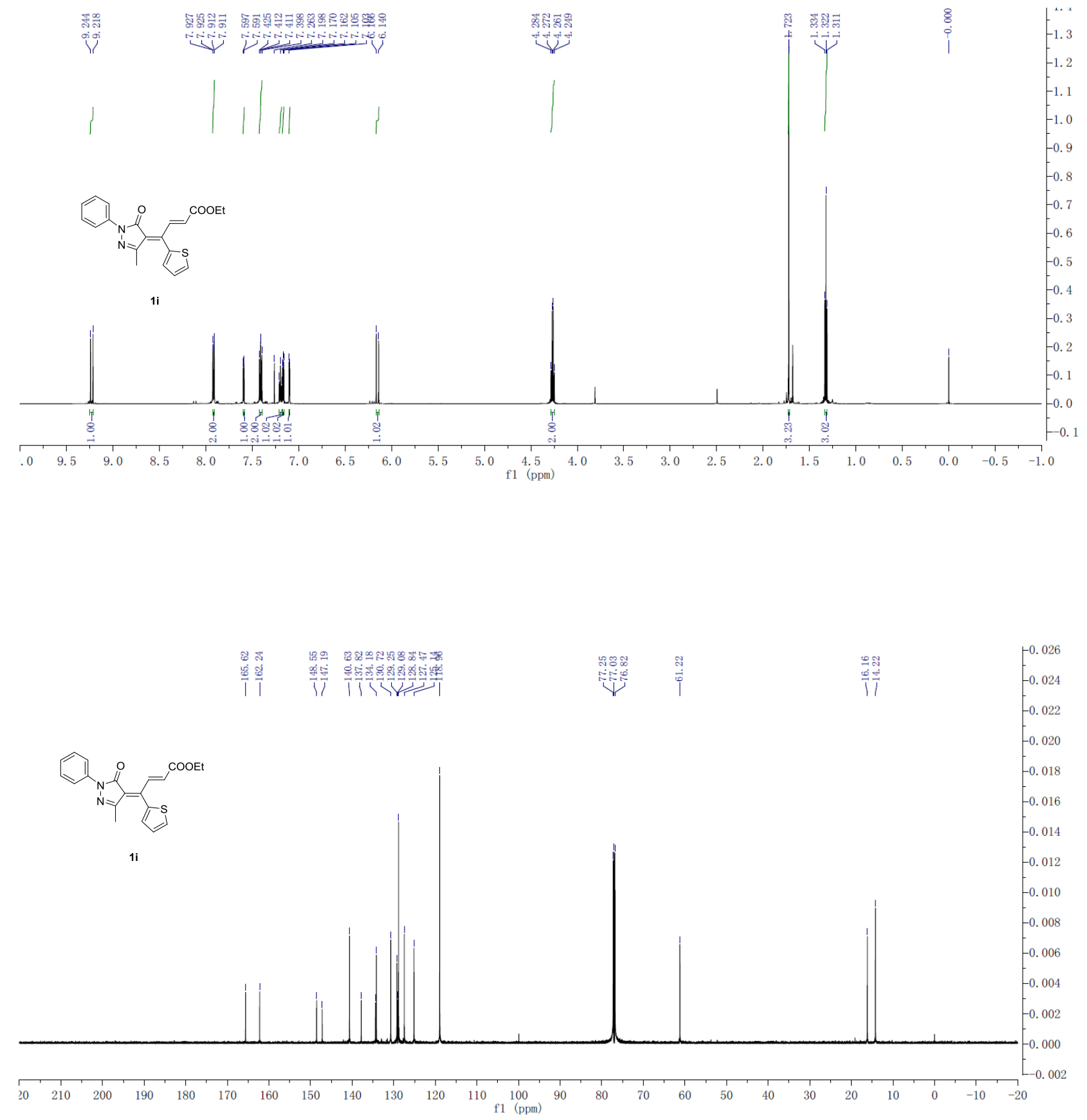


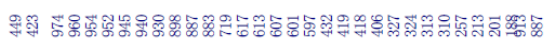

of

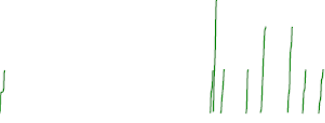

21

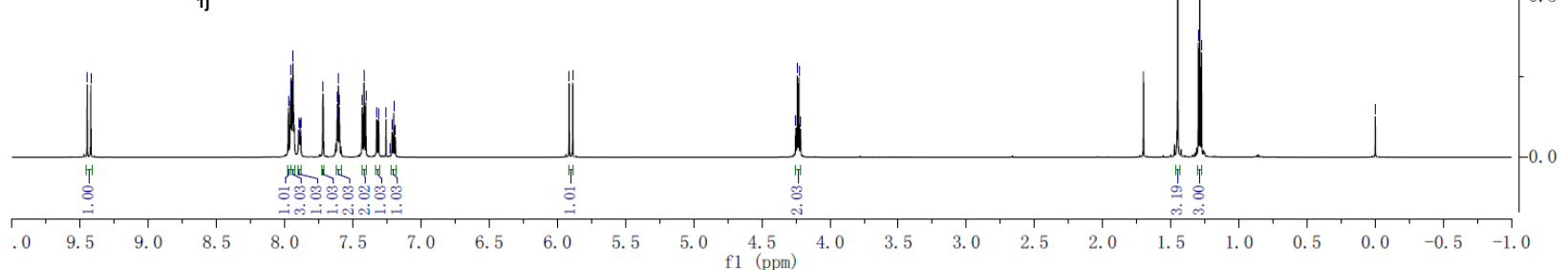

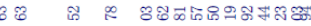

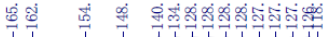

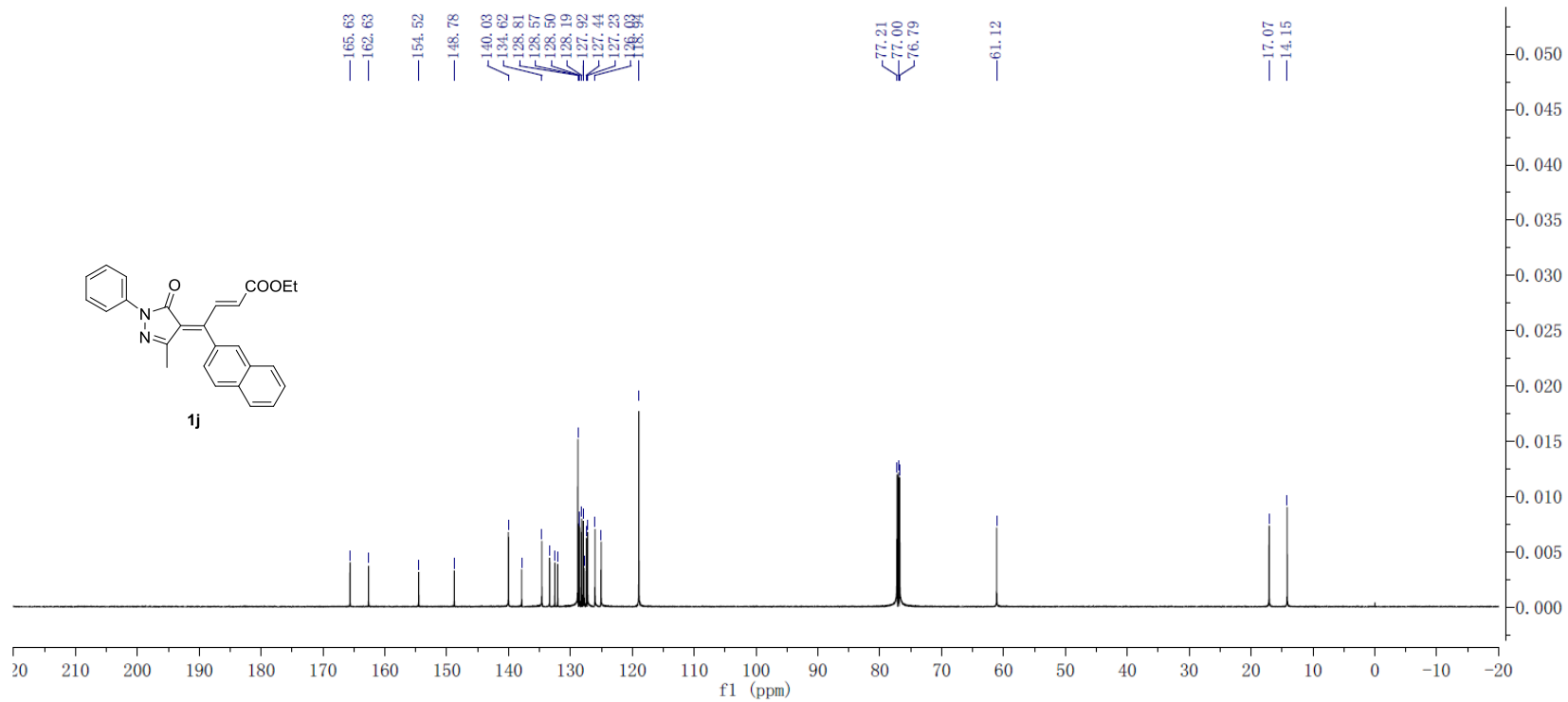



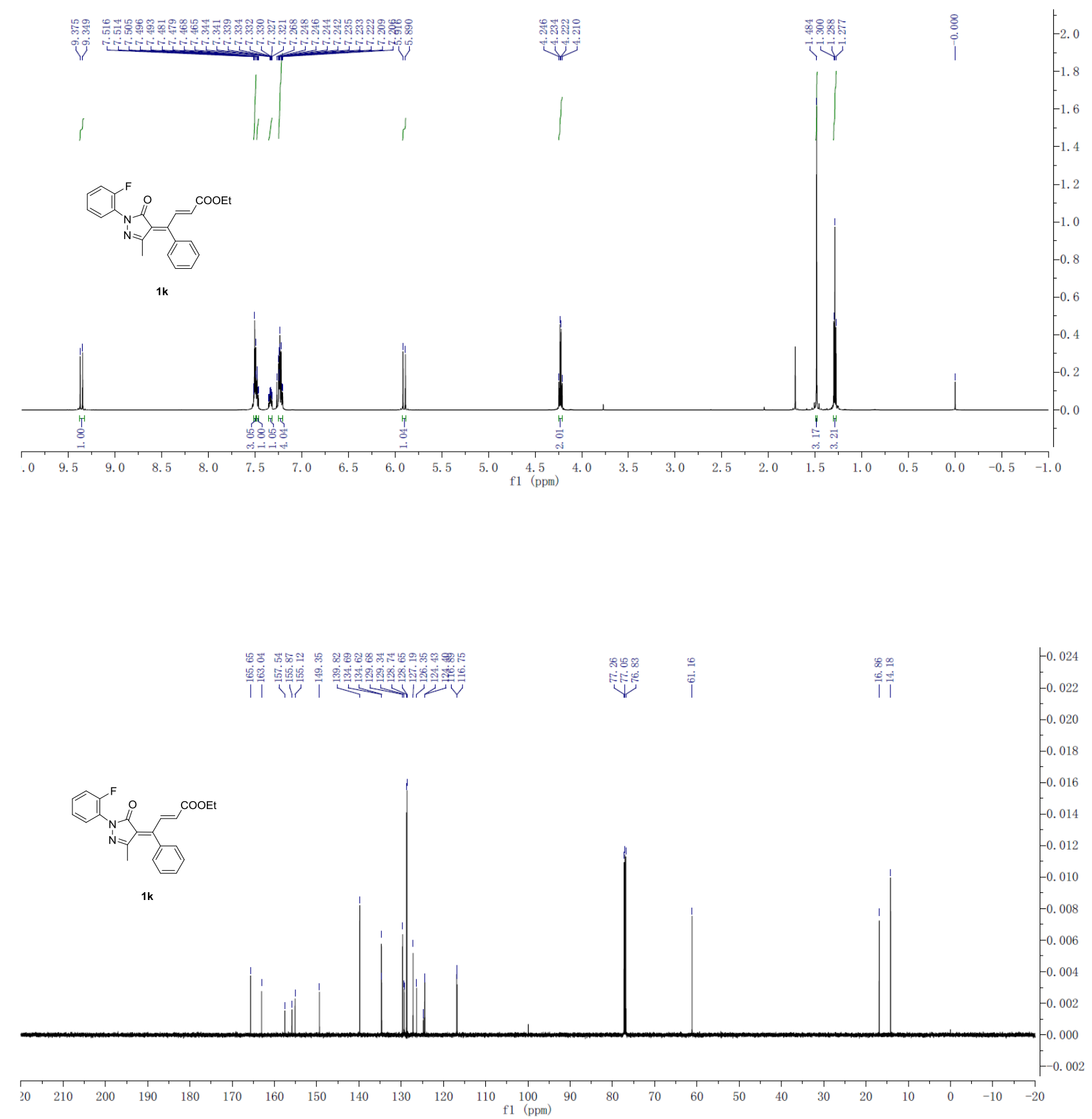


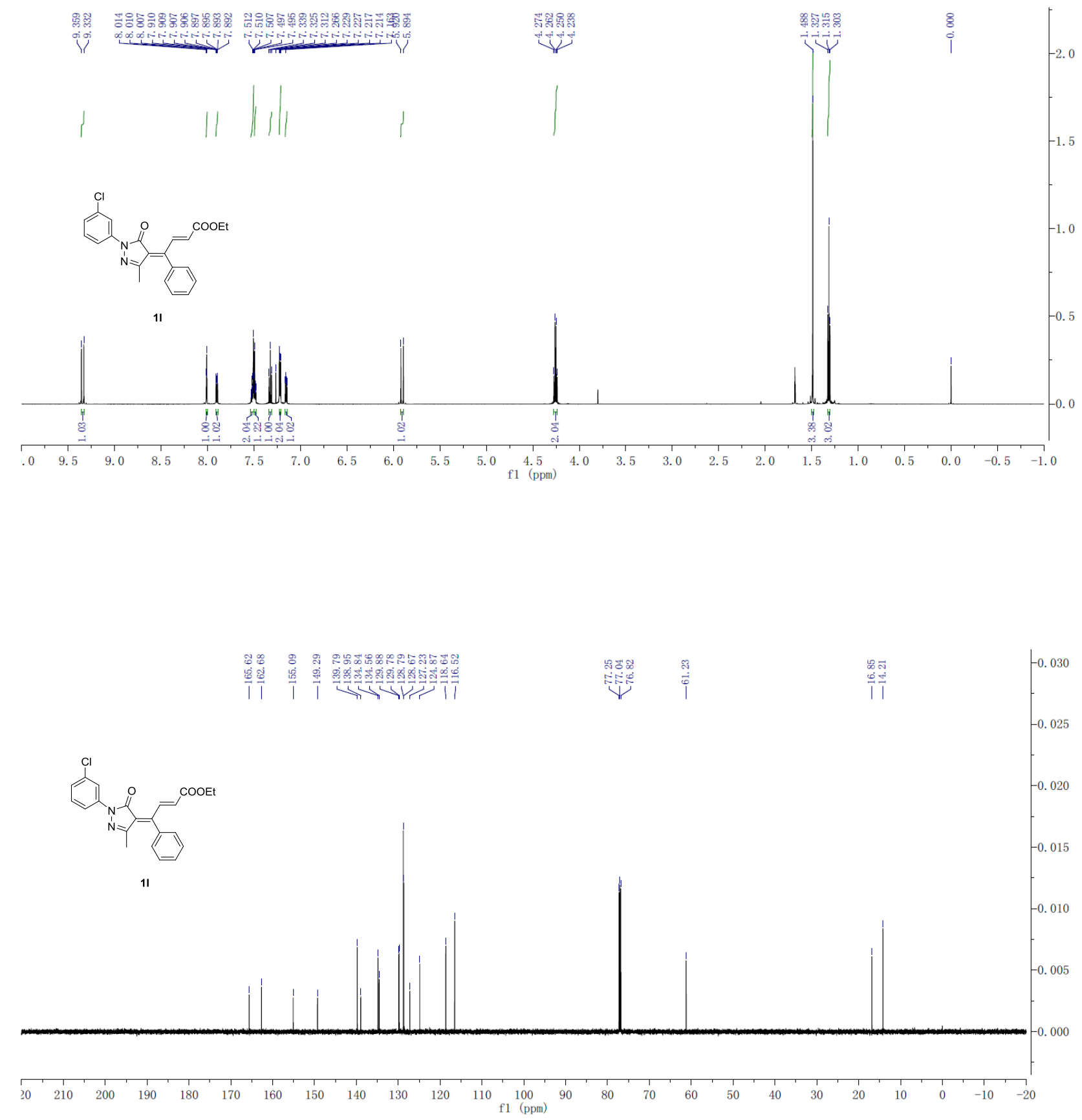




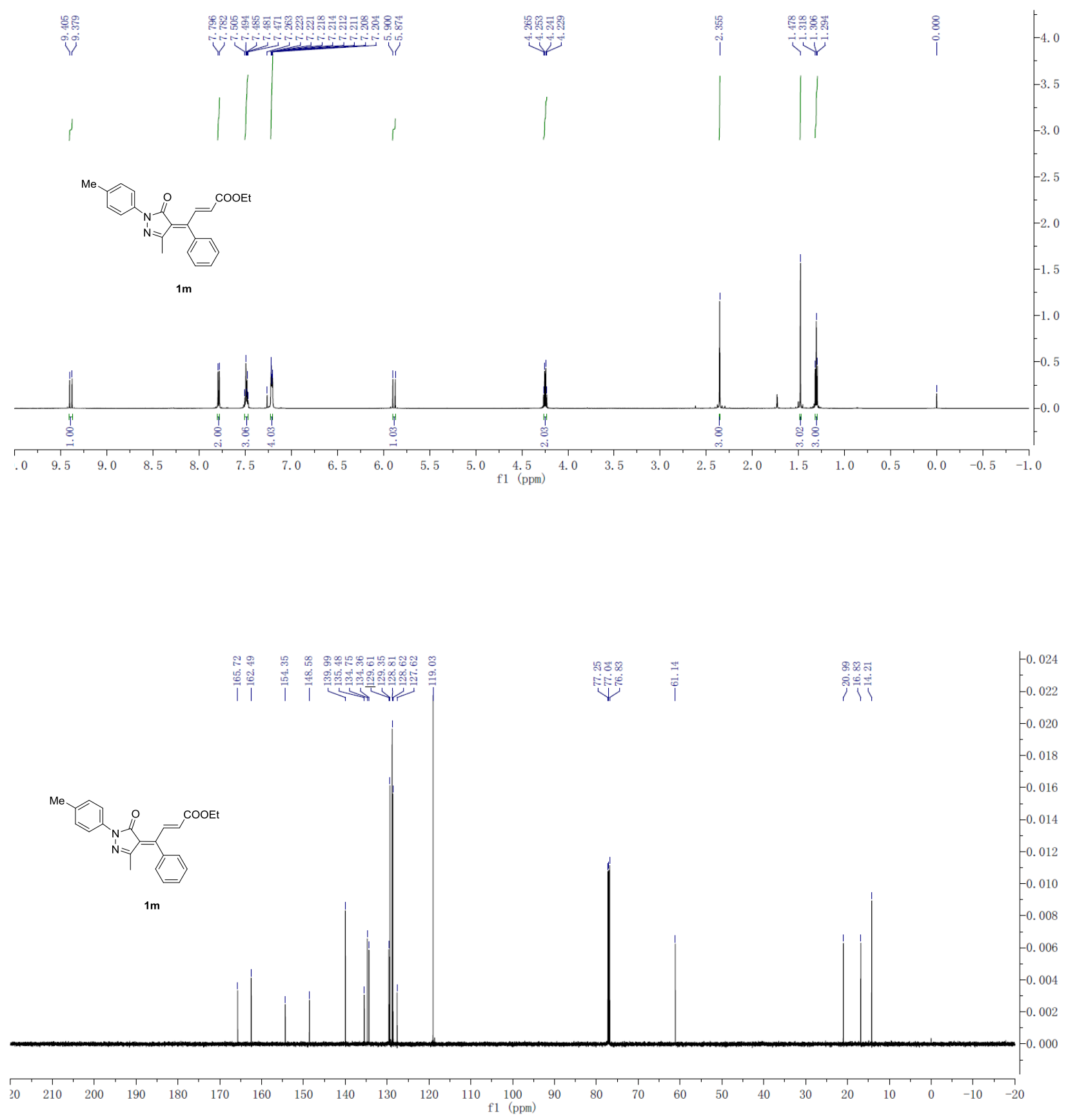



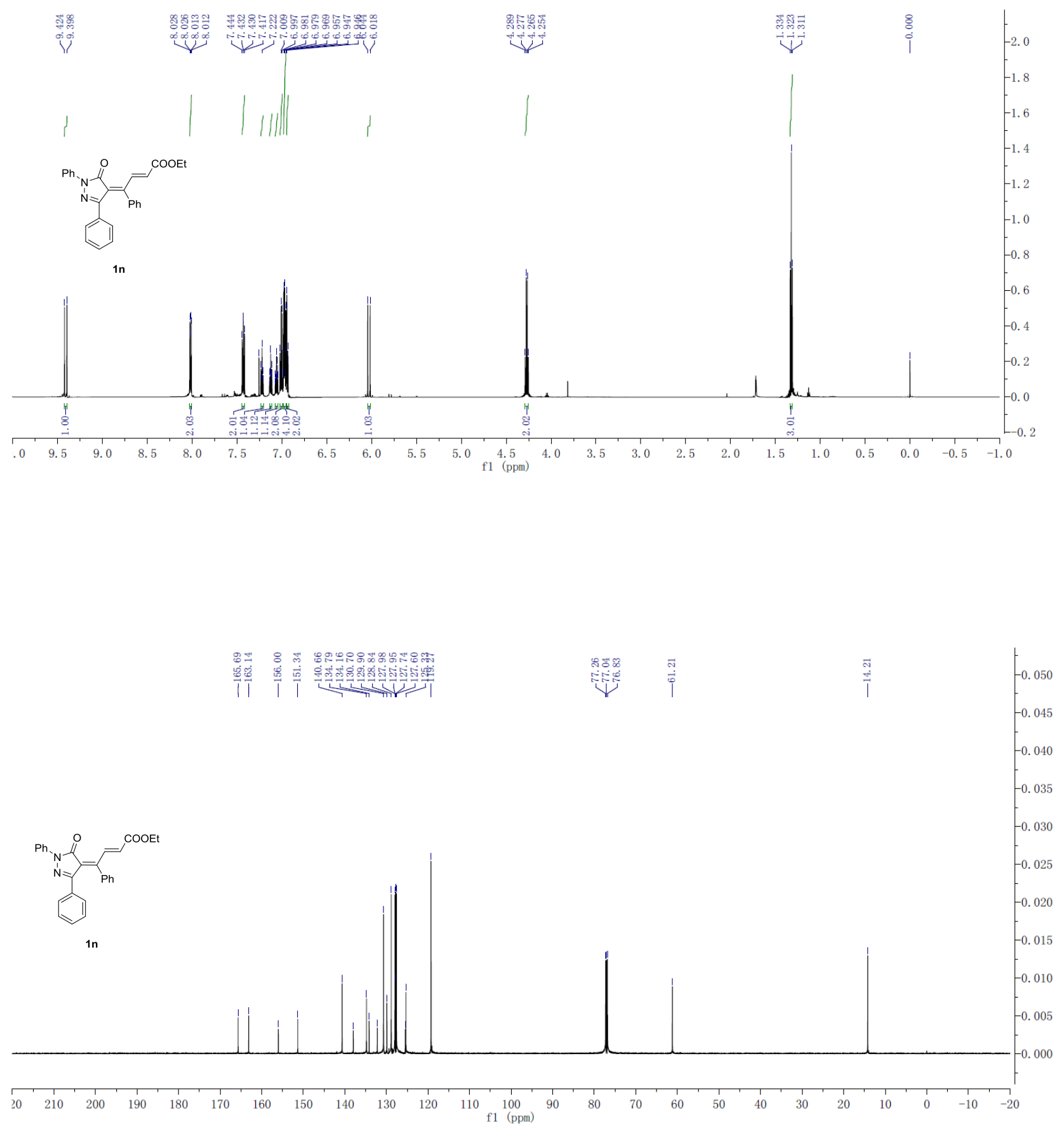

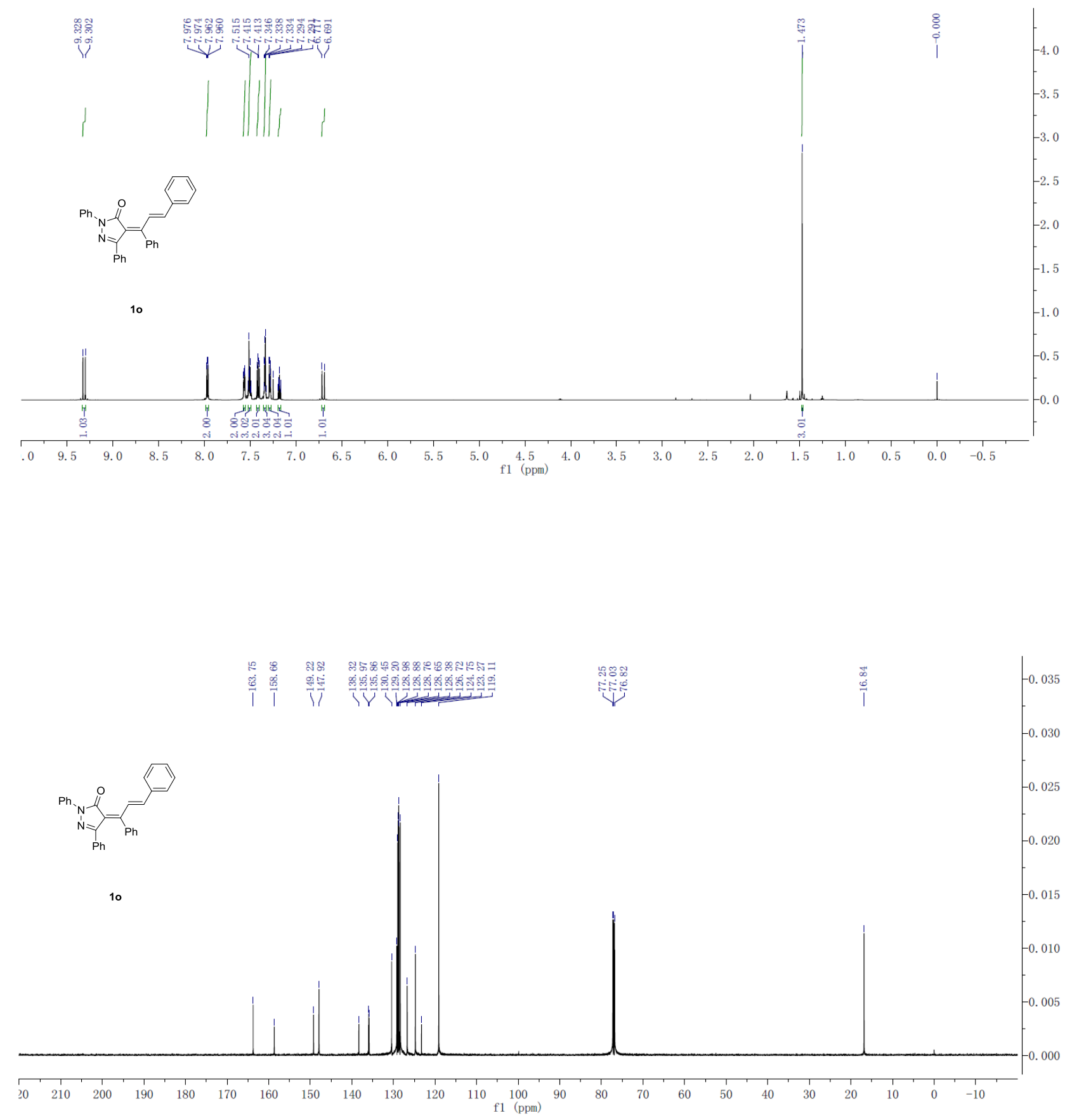

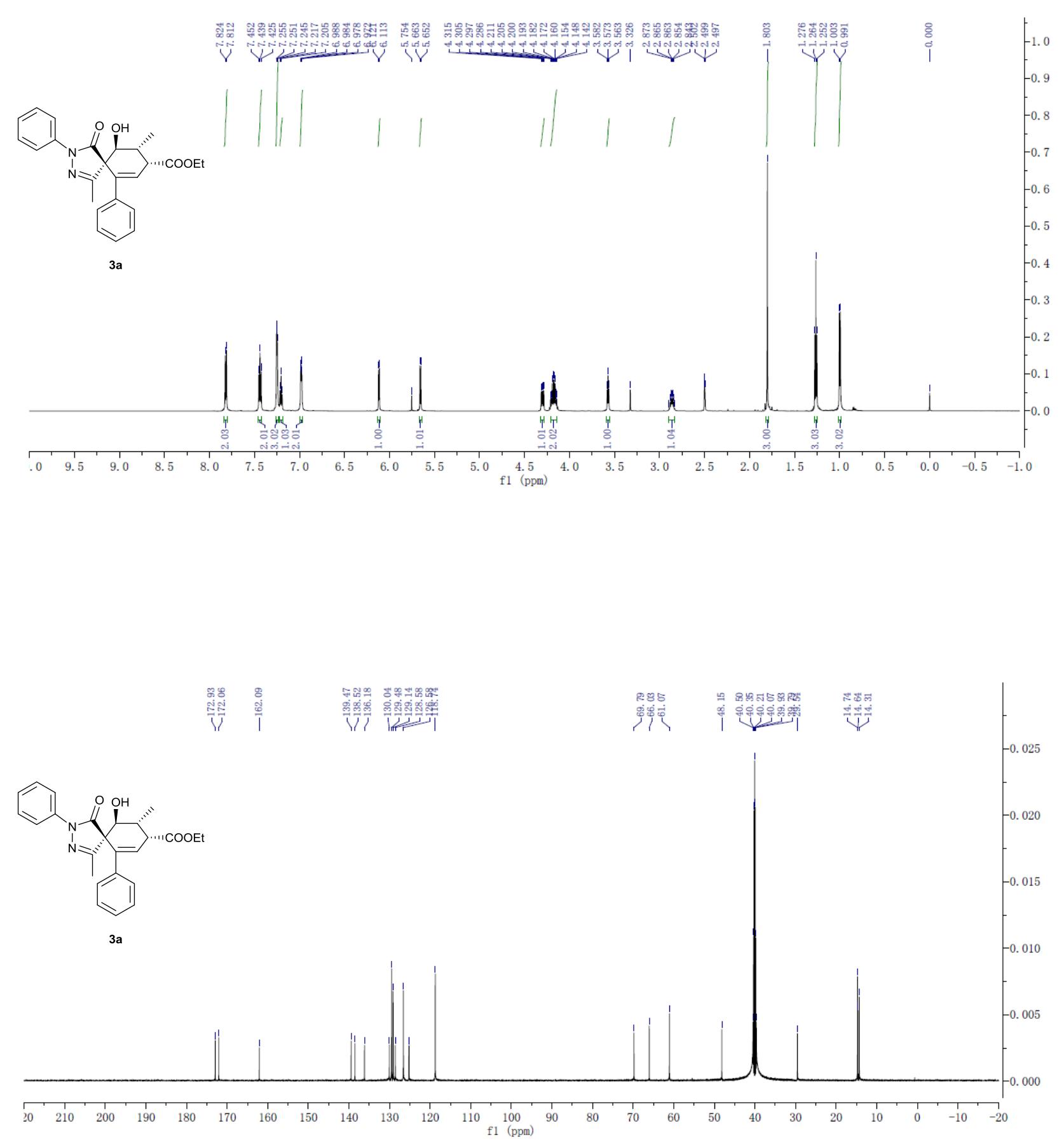

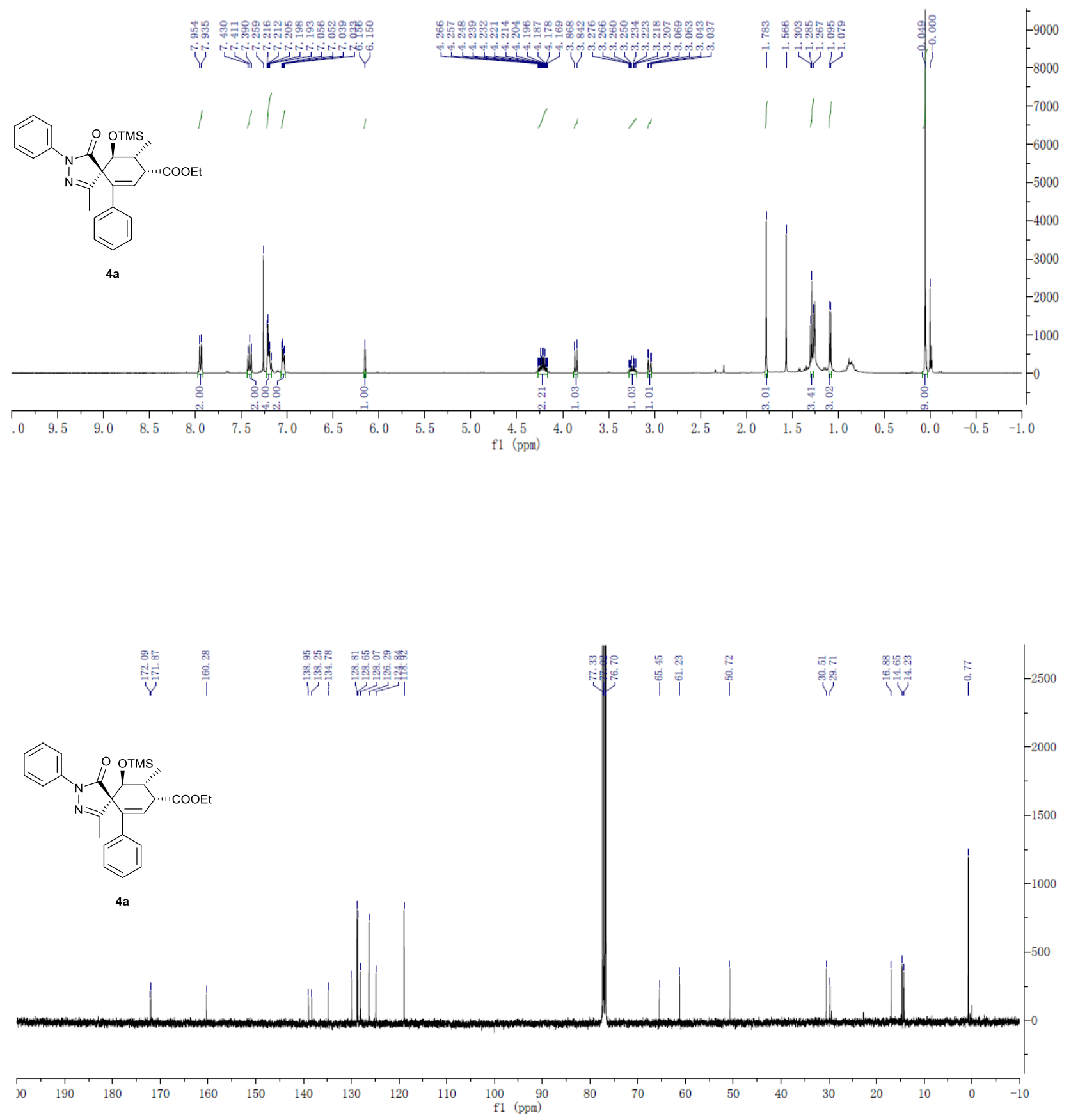


\section{Peak Analysis Report}

Detector A Channel $2254 \mathrm{~nm}$

\begin{tabular}{|c|c|c|c|c|}
\hline No. & Ret. Time & Height $(\mathrm{mAu})$ & Area $\left(\mathrm{mAu}^{*} \min \right)$ & Rel. Area (\%) \\
\hline 1 & 5.169 & 182987 & 1593958 & 50.123 \\
\hline 2 & 11.990 & 77391 & 1586105 & 49.877 \\
\hline Total & & 260378 & 3180063 & 100.000 \\
\hline
\end{tabular}

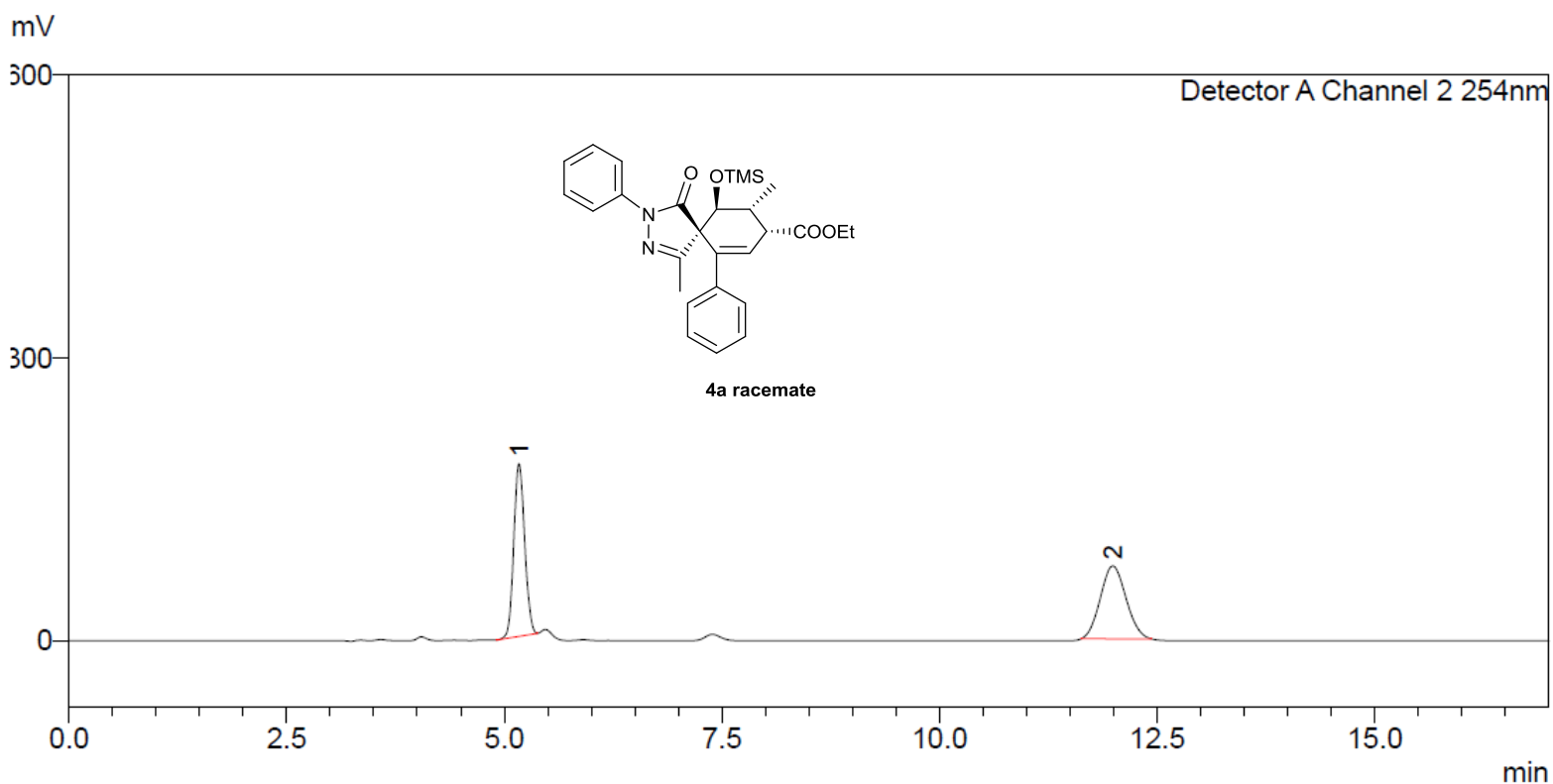

\section{Peak Analysis Report}

Detector A Channel 2 254nm

\begin{tabular}{|c|c|c|c|c|}
\hline No. & Ret. Time & Height $(\mathrm{mAu})$ & Area $\left(\mathrm{mAu}^{*} \mathrm{~min}\right)$ & Rel. Area $(\%)$ \\
\hline 1 & 5.037 & 25982 & 263104 & 2.050 \\
\hline 2 & 11.889 & 592176 & 12572471 & 97.950 \\
\hline Total & & 618158 & 12835575 & 100.000 \\
\hline
\end{tabular}

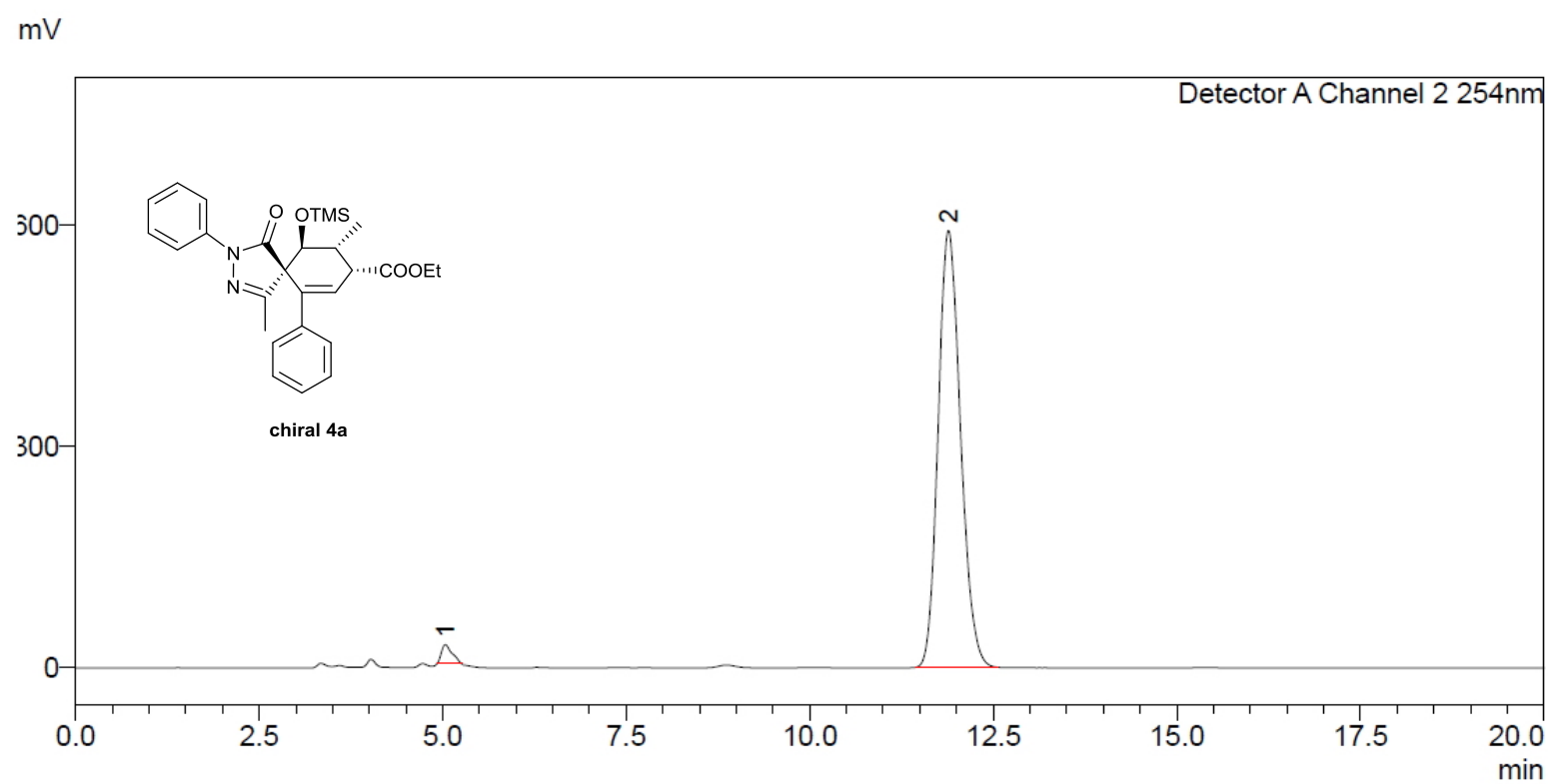



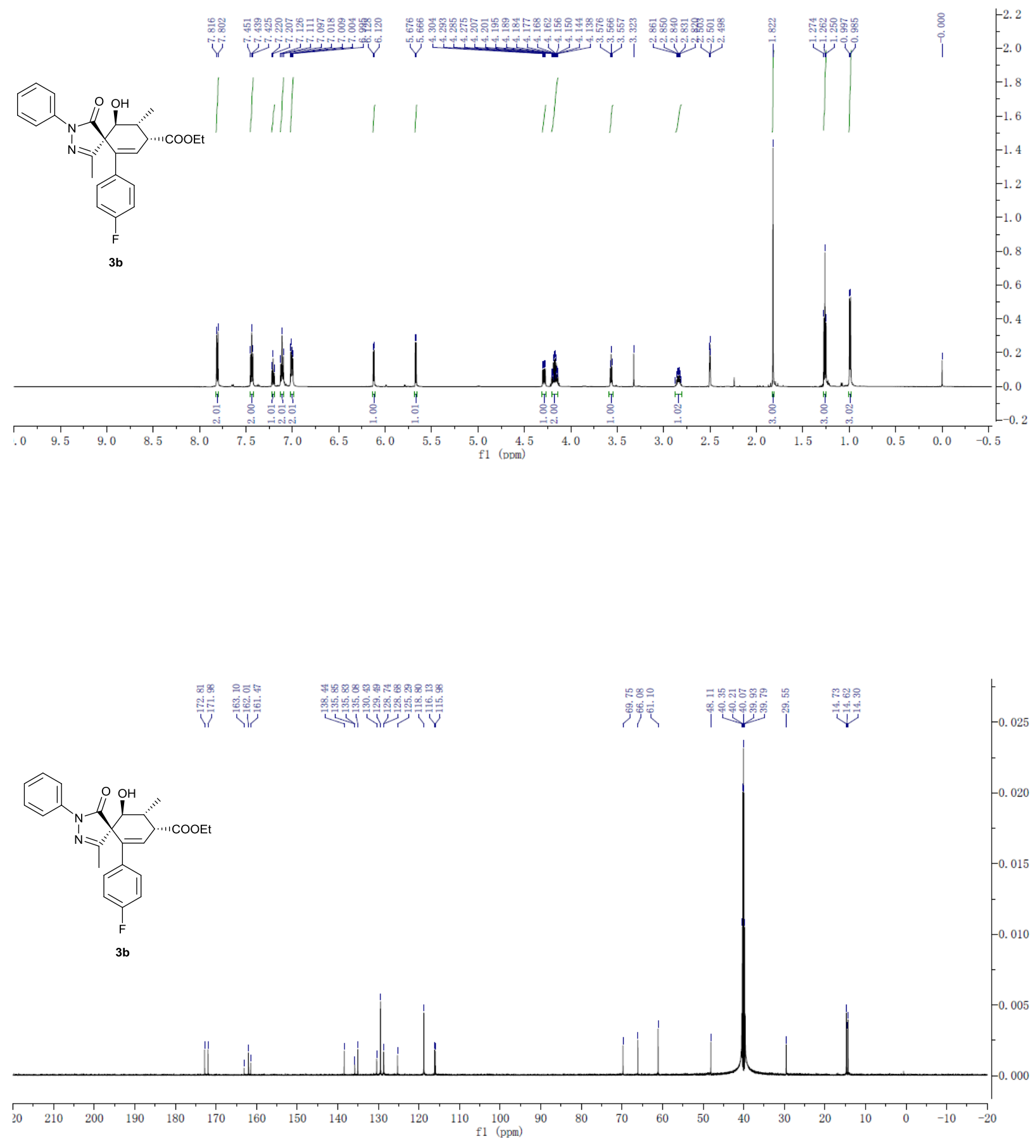


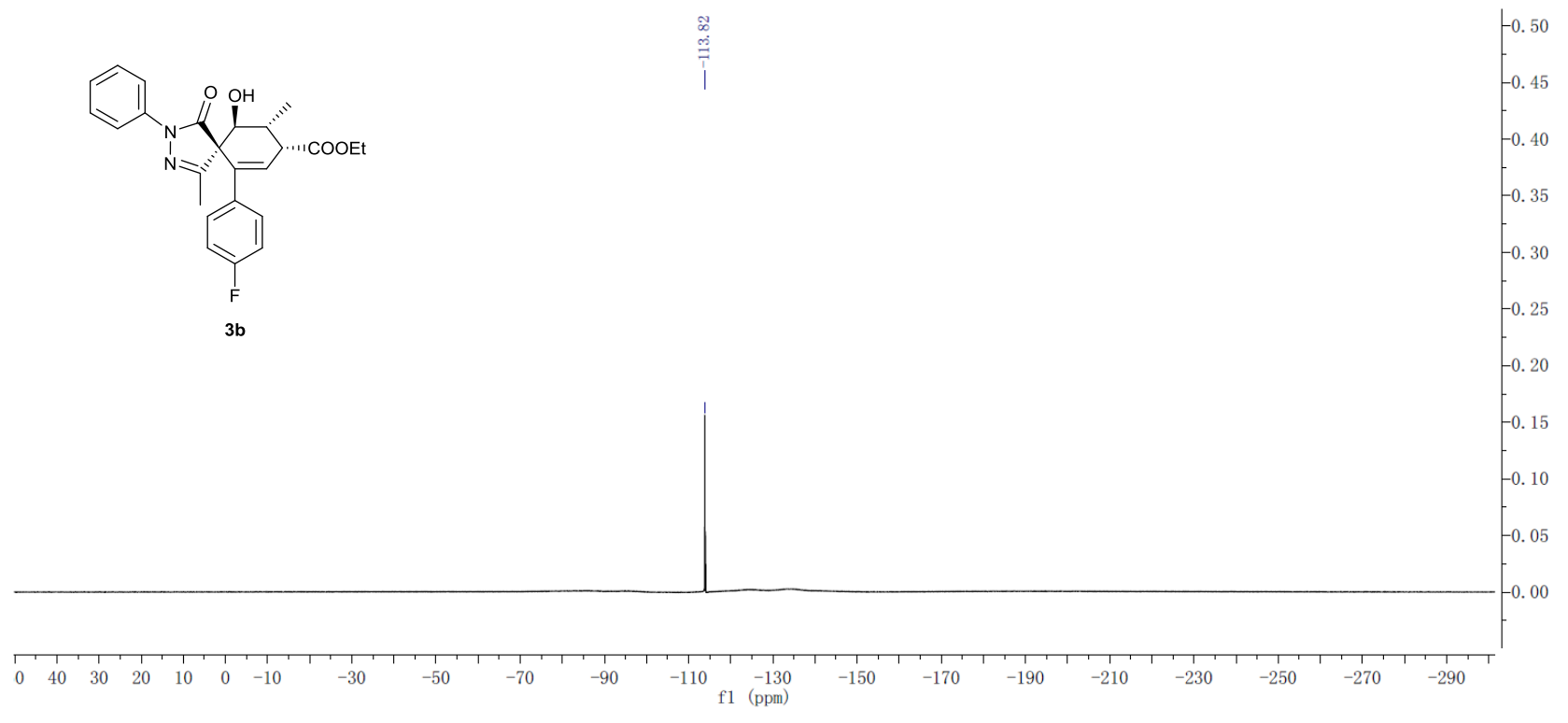



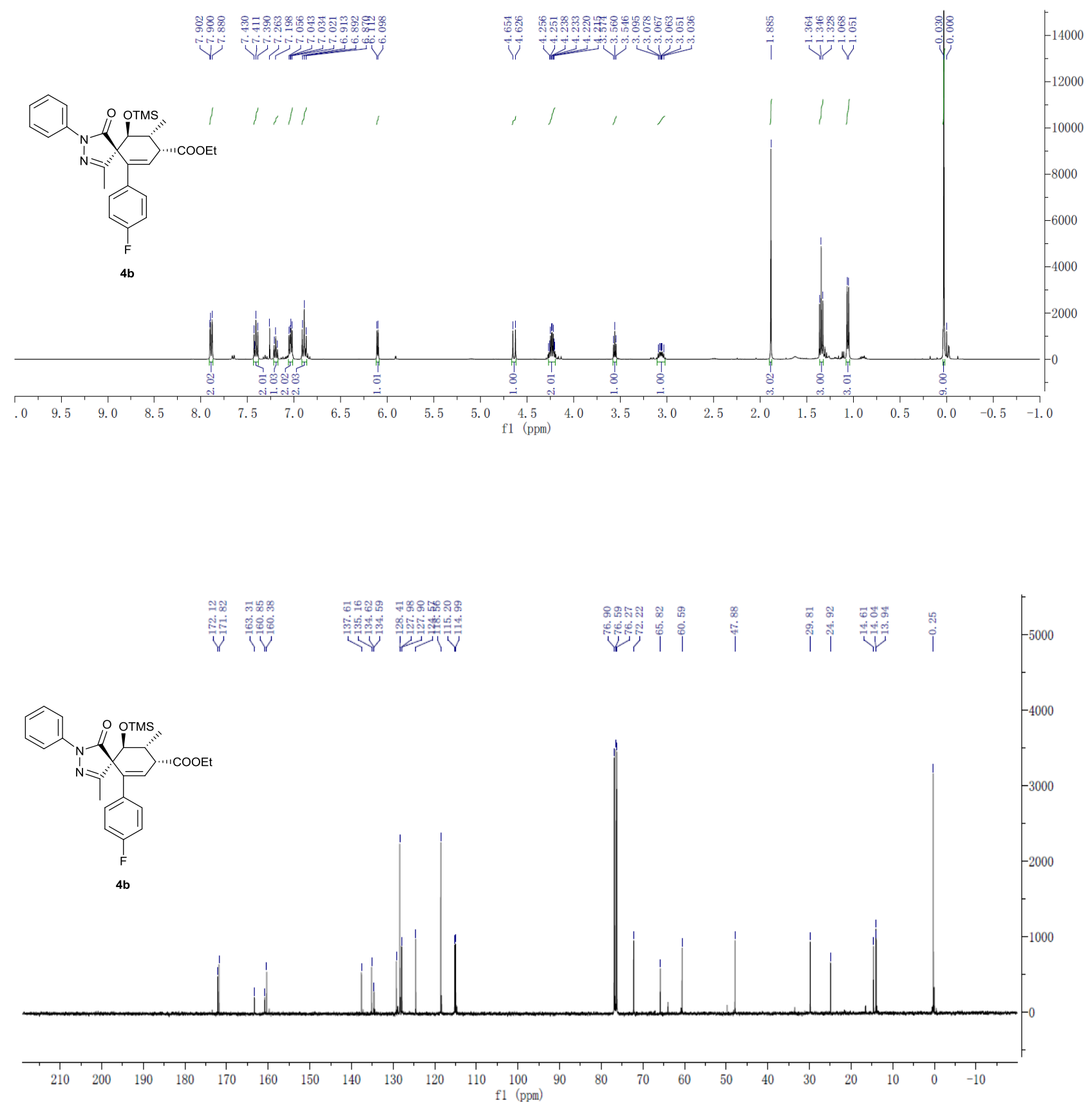


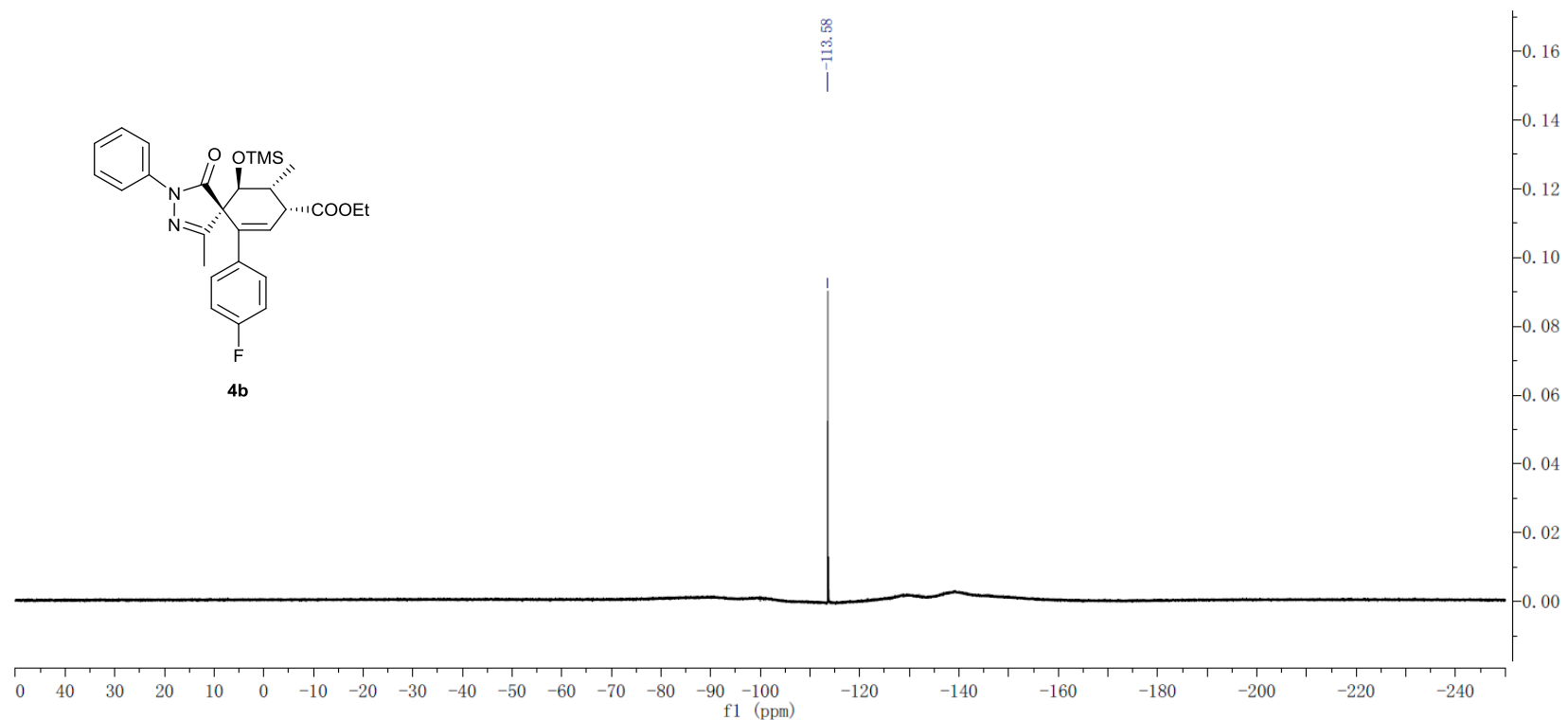




\section{Peak Analysis Report}

Detector A Channel 2 254nm

\begin{tabular}{|c|c|c|c|c|}
\hline No. & Ret. Time & Height $(\mathrm{mAu})$ & Area $\left(\mathrm{mAu}^{*} \mathrm{~min}\right)$ & Rel. Area $(\%)$ \\
\hline 1 & 11.260 & 923154 & 14454926 & 50.763 \\
\hline 2 & 18.440 & 542922 & 14020556 & 49.237 \\
\hline Total & & 1466076 & 28475482 & 100.000 \\
\hline
\end{tabular}

$\mathrm{mV}$

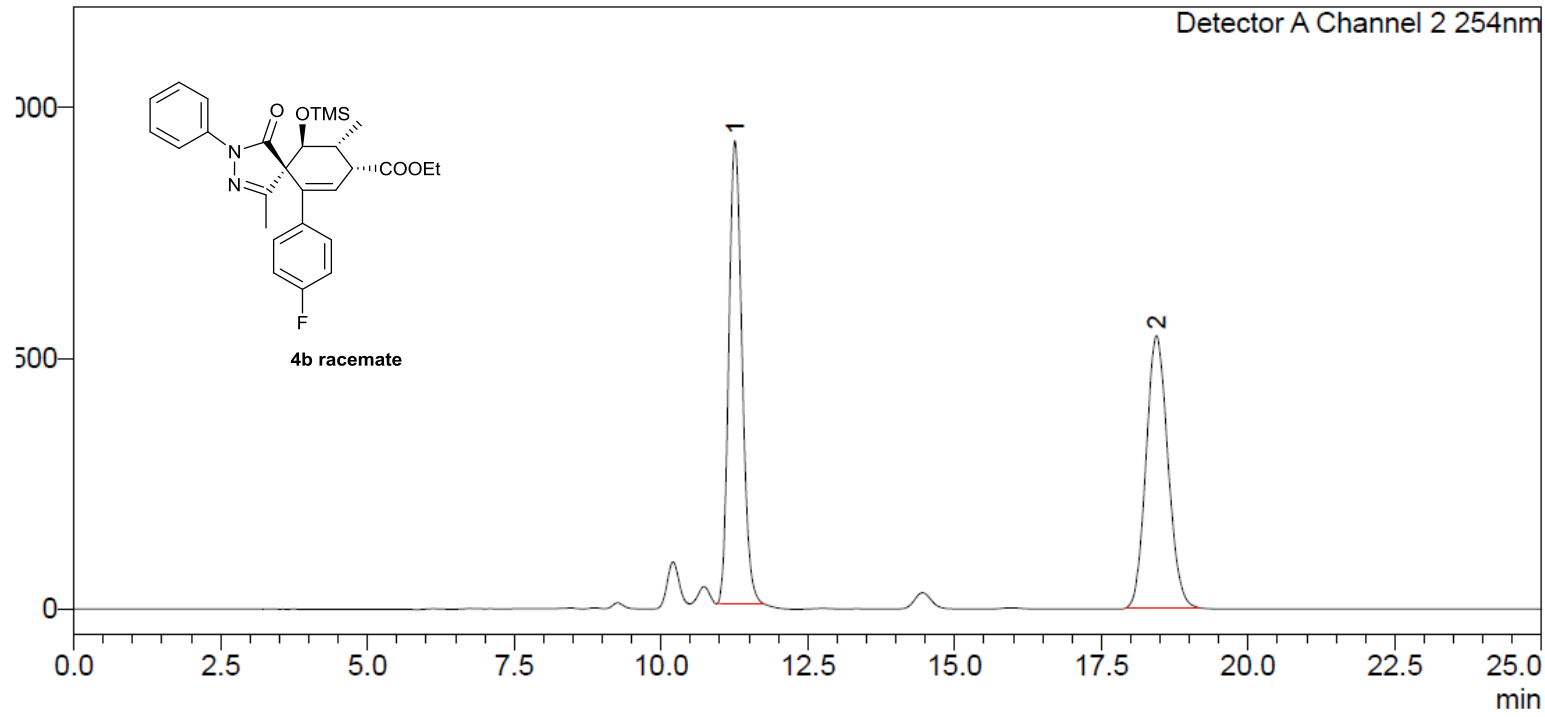

\section{Peak Analysis Report}

Detector A Channel $2254 \mathrm{~nm}$

\begin{tabular}{|c|c|c|c|c|}
\hline No. & Ret. Time & Height $(\mathrm{mAu})$ & Area $\left(\mathrm{mAu}^{*} \mathrm{~min}\right)$ & Rel. Area $(\%)$ \\
\hline 1 & 11.146 & 30780 & 465526 & 3.027 \\
\hline 2 & 18.162 & 579049 & 14916062 & 96.973 \\
\hline Total & & 609828 & 15381588 & 100.000 \\
\hline
\end{tabular}

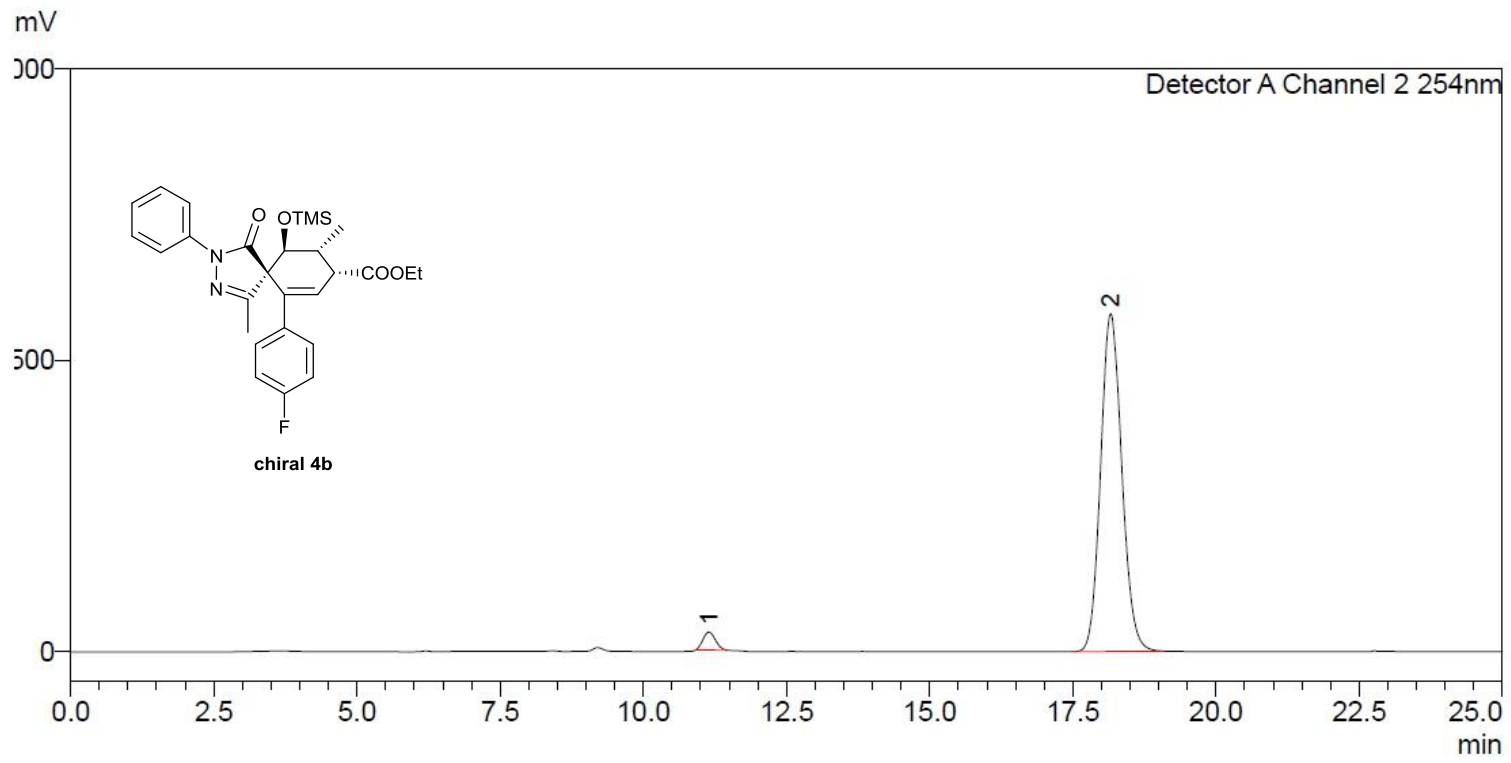



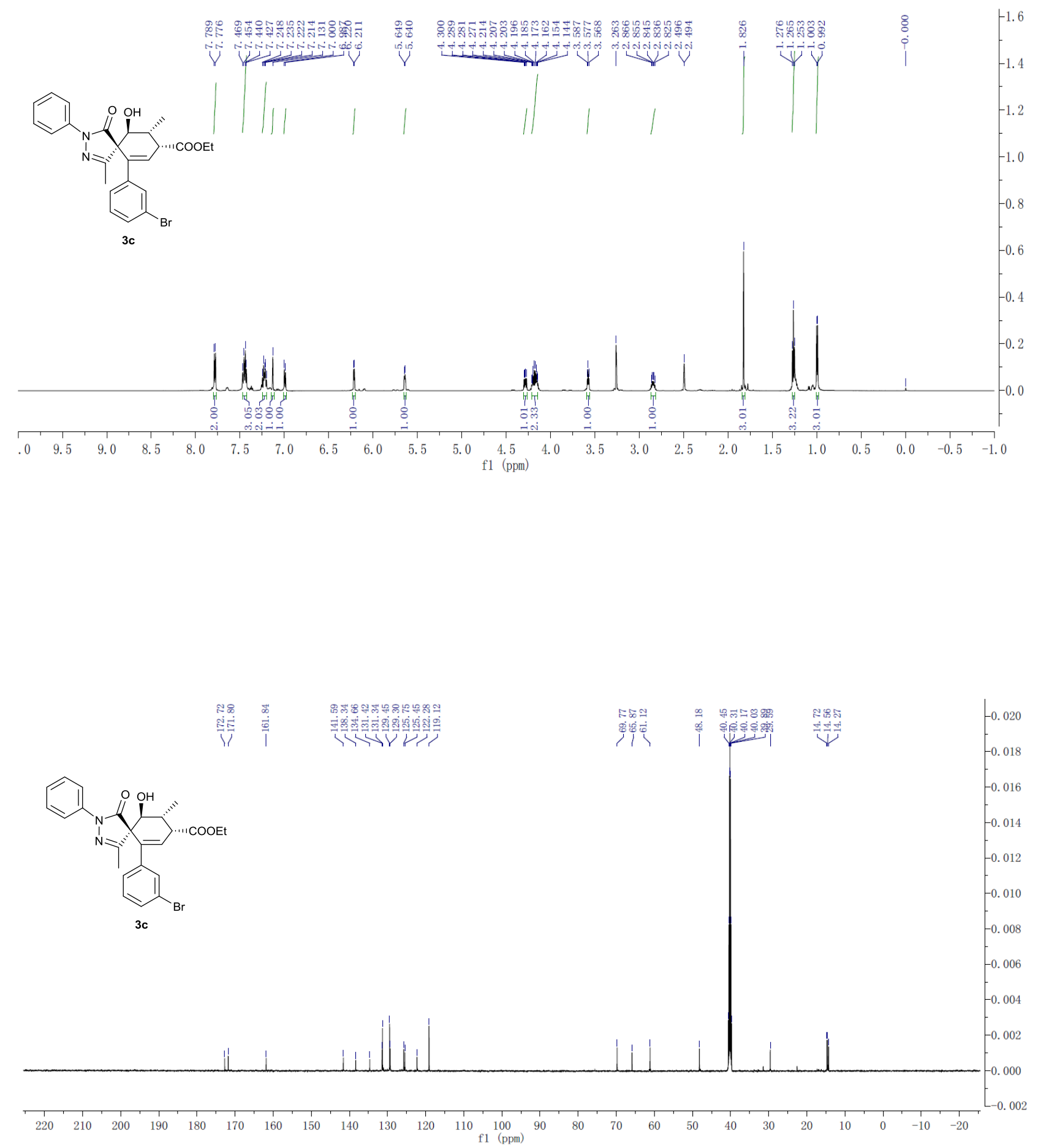

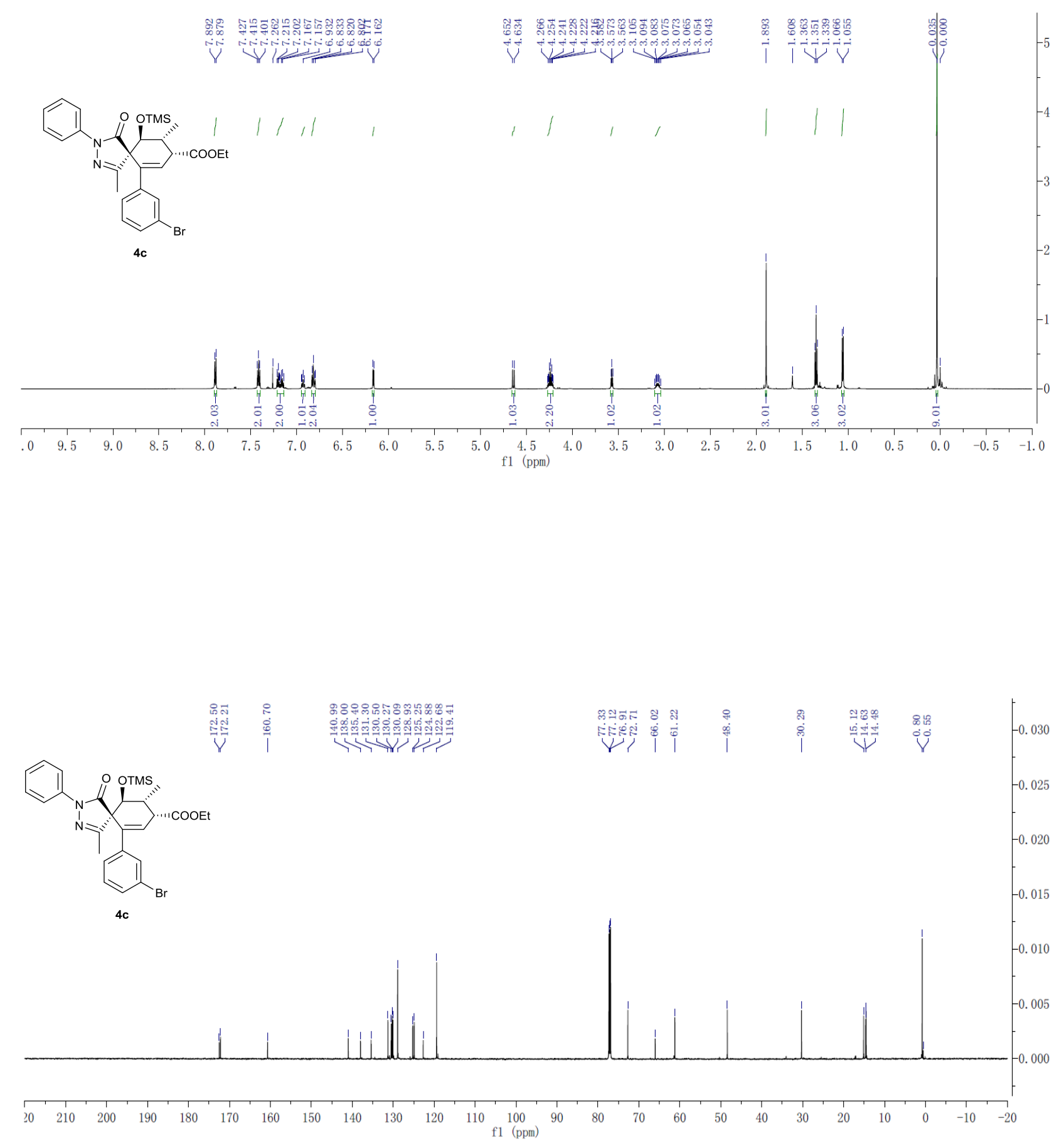


\section{Peak Analysis Report}

Detector A Channel 2 254nm

\begin{tabular}{|c|c|c|c|c|}
\hline No. & Ret. Time & Height $(\mathrm{mAu})$ & Area $\left(\mathrm{mAu}^{*} \min \right)$ & Rel. Area (\%) \\
\hline 1 & 10.835 & 422545 & 7307583 & 49.405 \\
\hline 2 & 18.114 & 248226 & 7483612 & 50.595 \\
\hline Total & & 670771 & 14791195 & 100.000 \\
\hline
\end{tabular}

$\mathrm{mV}$

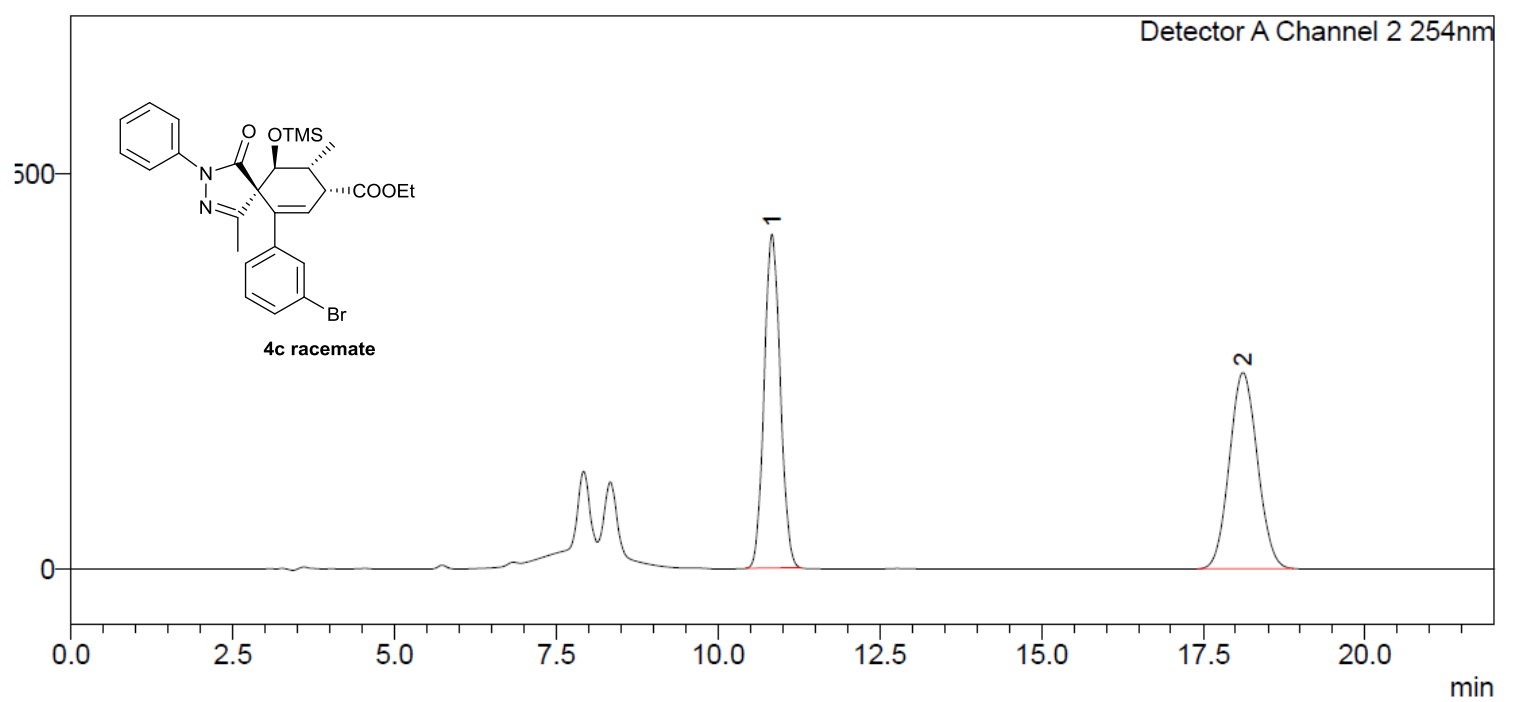

\section{Peak Analysis Report}

Detector A Channel 2 254nm

\begin{tabular}{|c|c|c|c|c|}
\hline No. & Ret. Time & Height $(\mathrm{mAu})$ & Area $\left(\mathrm{mAu}^{*} \mathrm{~min}\right)$ & Rel. Area $(\%)$ \\
\hline 1 & 10.803 & 16125 & 256815 & 1.071 \\
\hline 2 & 17.997 & 773577 & 23717599 & 98.929 \\
\hline Total & & 789702 & 23974414 & 100.000 \\
\hline
\end{tabular}

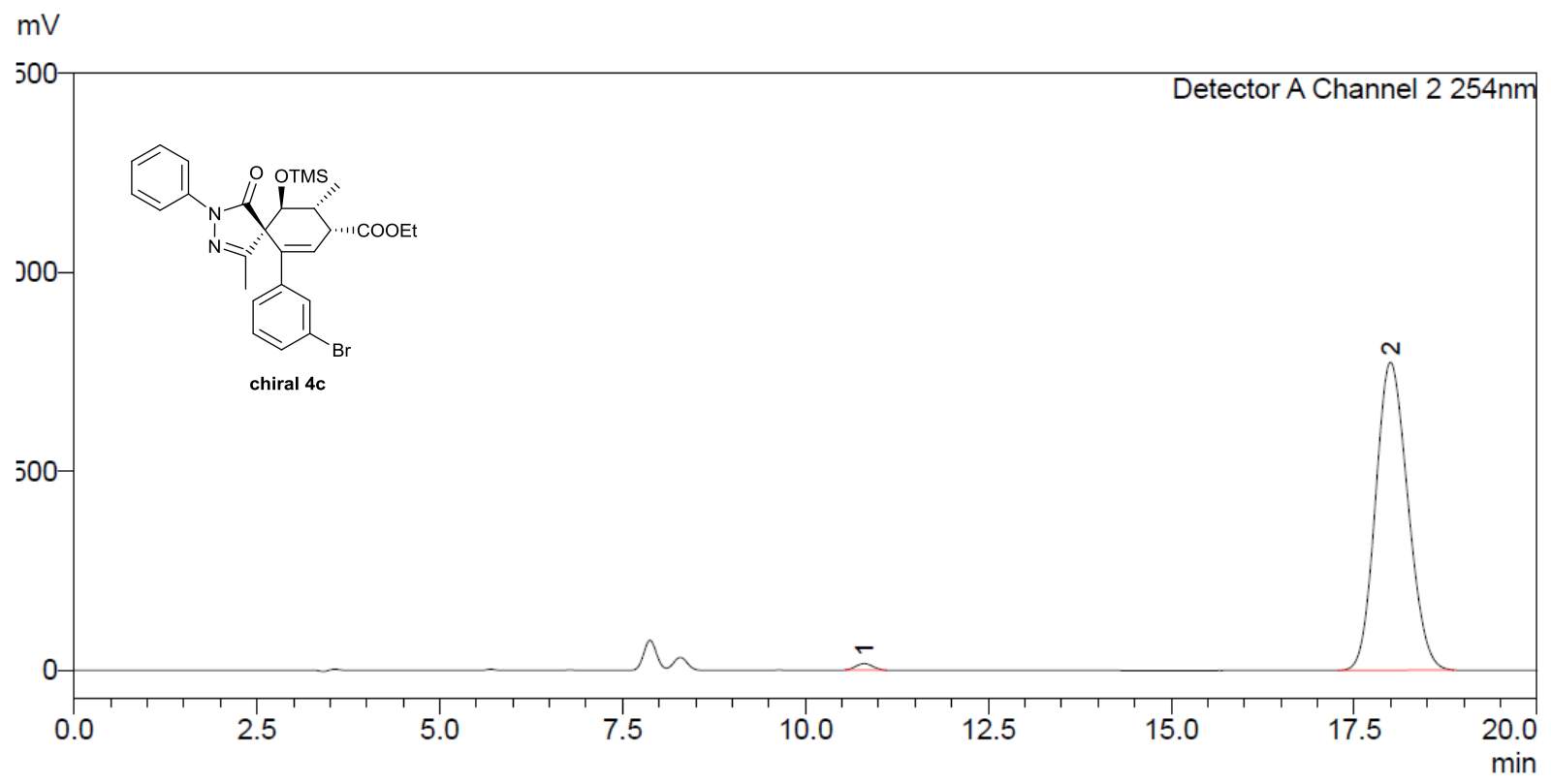



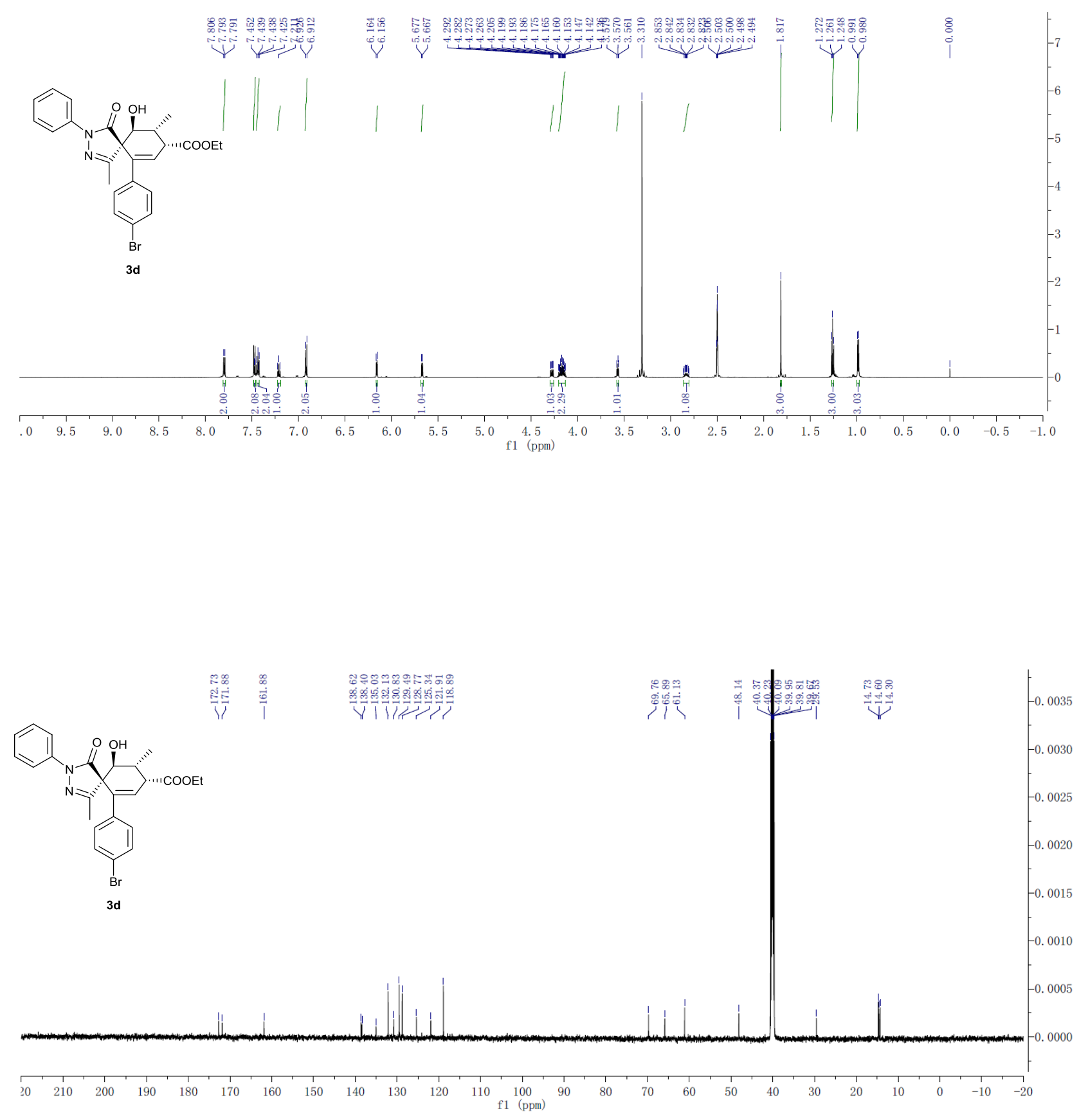

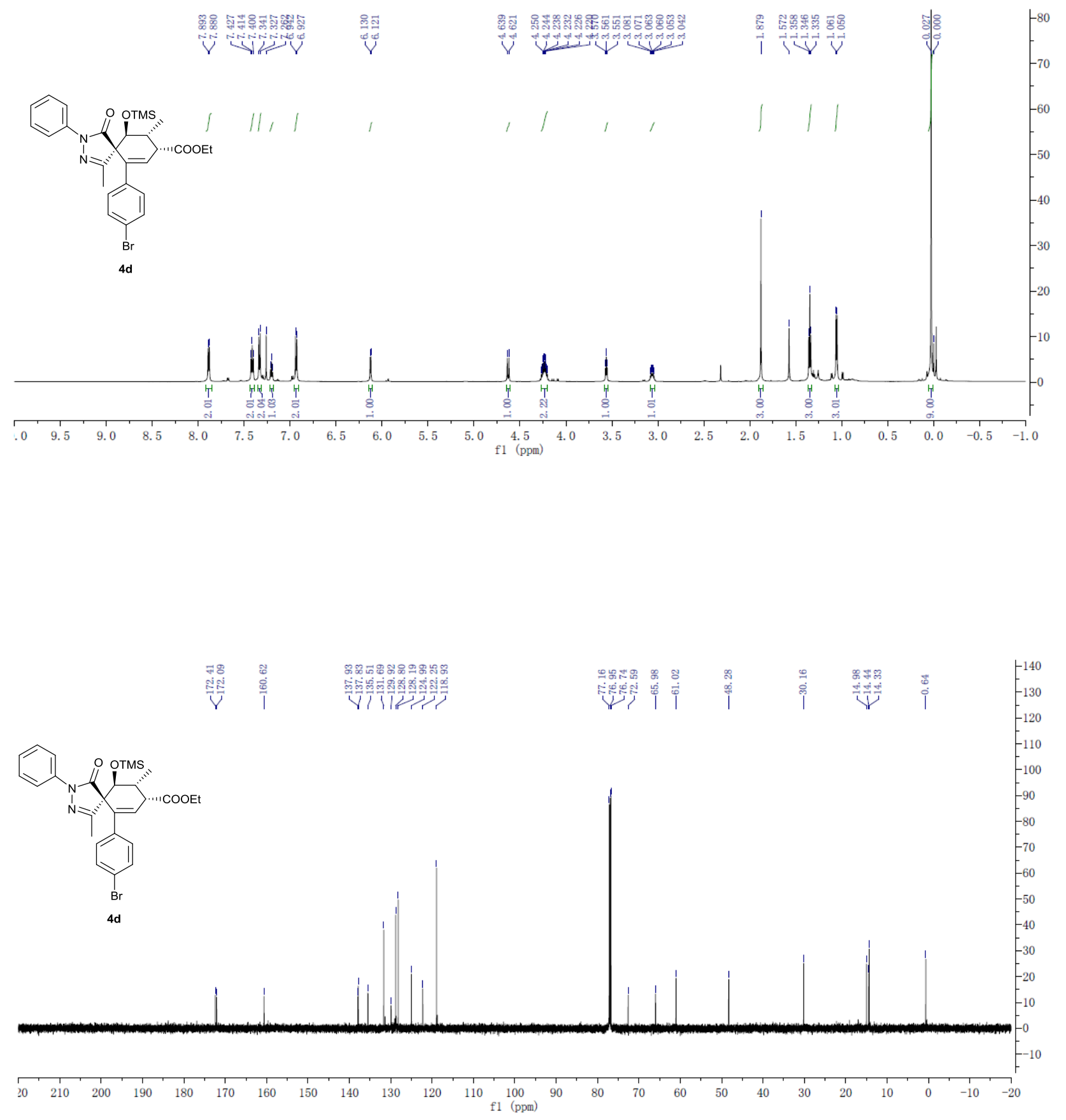


\section{Peak Analysis Report}

Detector A Channel $2254 \mathrm{~nm}$

\begin{tabular}{|c|c|c|c|c|}
\hline No. & Ret. Time & Height $(\mathrm{mAu})$ & Area $\left(\mathrm{mAu}^{*} \mathrm{~min}\right)$ & Rel. Area $(\%)$ \\
\hline 1 & 6.465 & 432807 & 4551080 & 49.532 \\
\hline 2 & 11.997 & 213552 & 4637124 & 50.468 \\
\hline Total & & 646359 & 9188204 & 100.000 \\
\hline
\end{tabular}

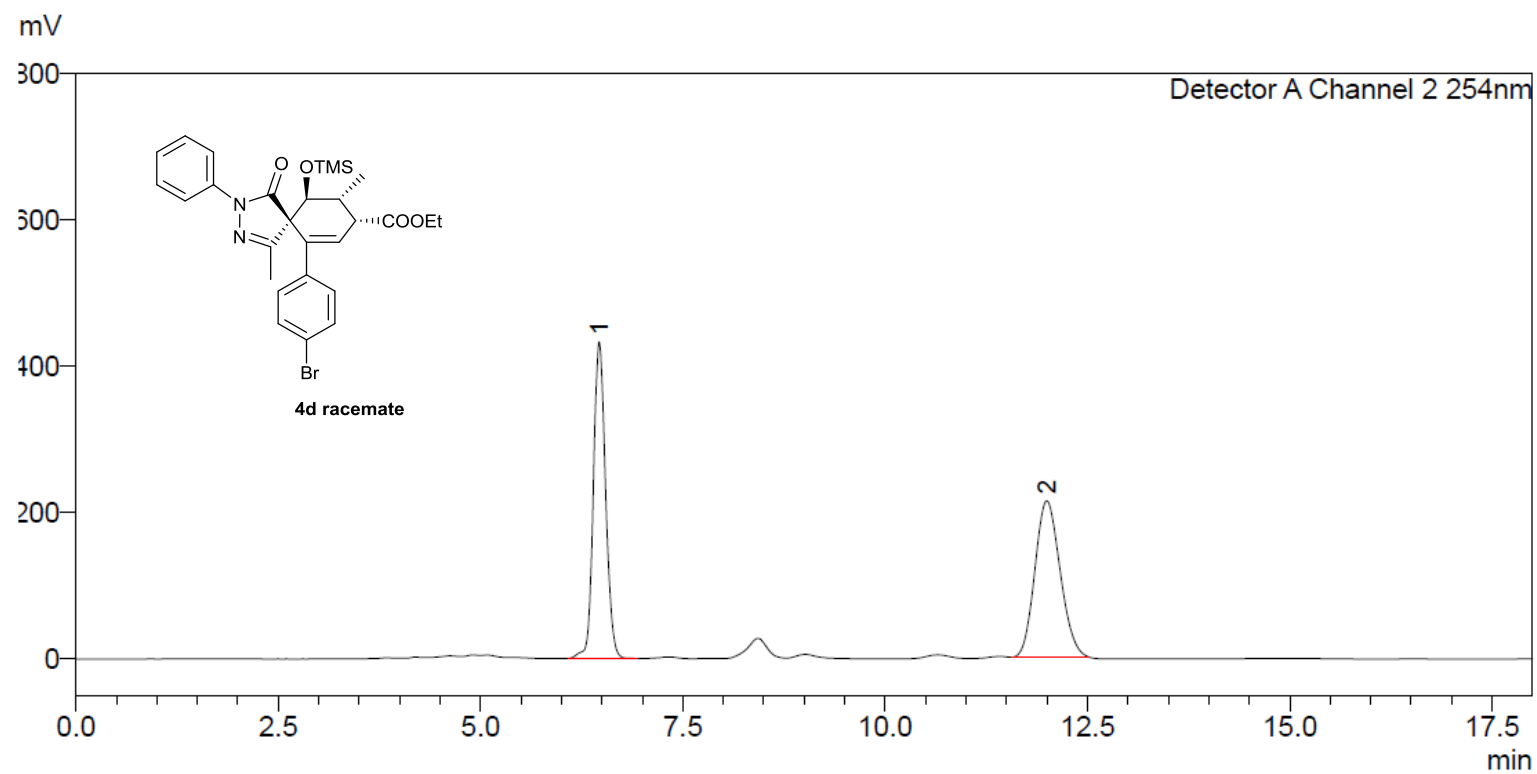

\section{Peak Analysis Report}

Detector A Channel $2254 \mathrm{~nm}$
\begin{tabular}{|c|c|c|c|c|}
\hline No. & Ret. Time & Height (mAu) & Area (mAu*min) & Rel. Area $(\%)$ \\
\hline 1 & 5.882 & 23599 & 229069 & 1.294 \\
\hline 2 & 11.798 & 790342 & 17470673 & 98.706 \\
\hline Total & & 813941 & 17699743 & 100.000 \\
\hline
\end{tabular}

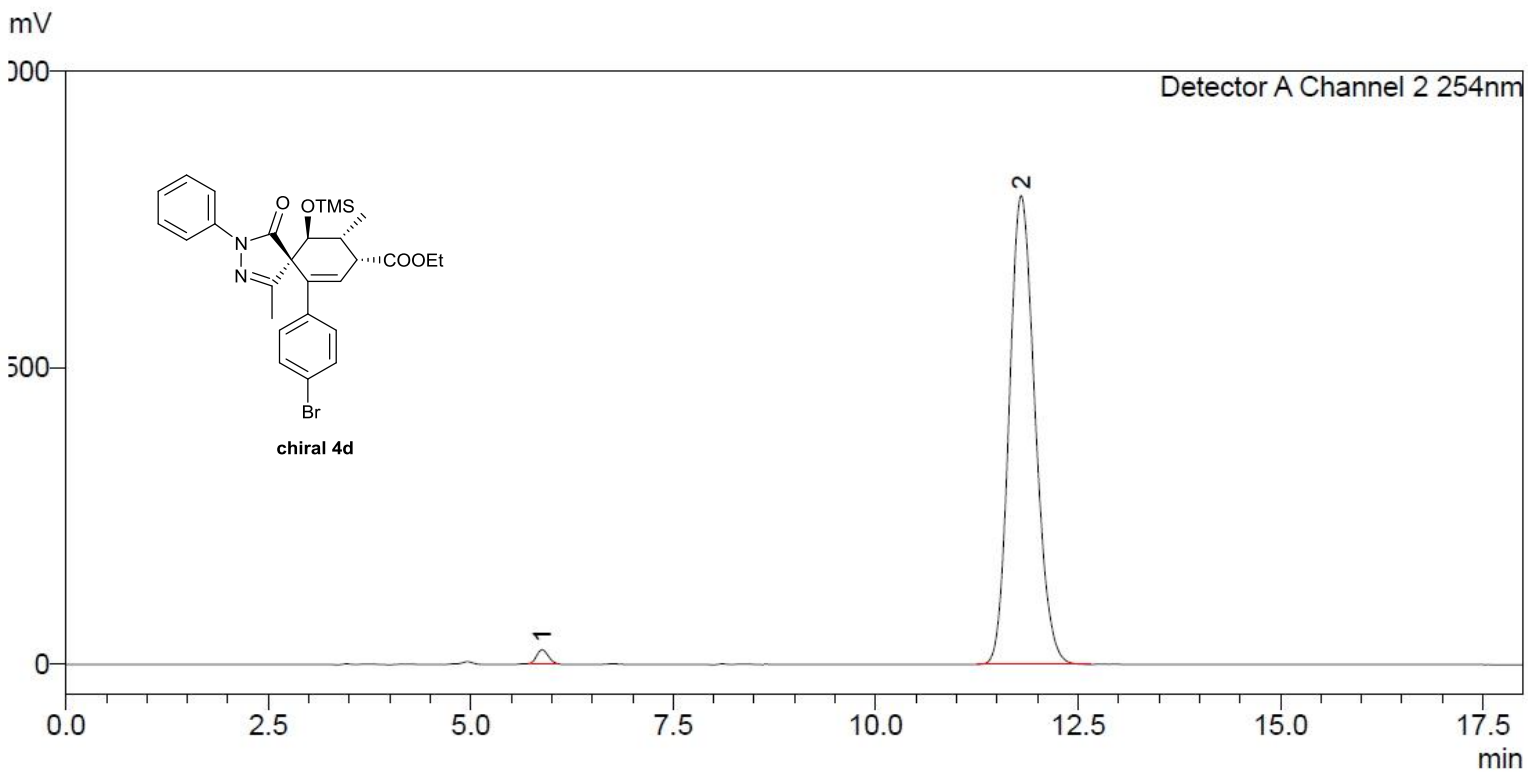



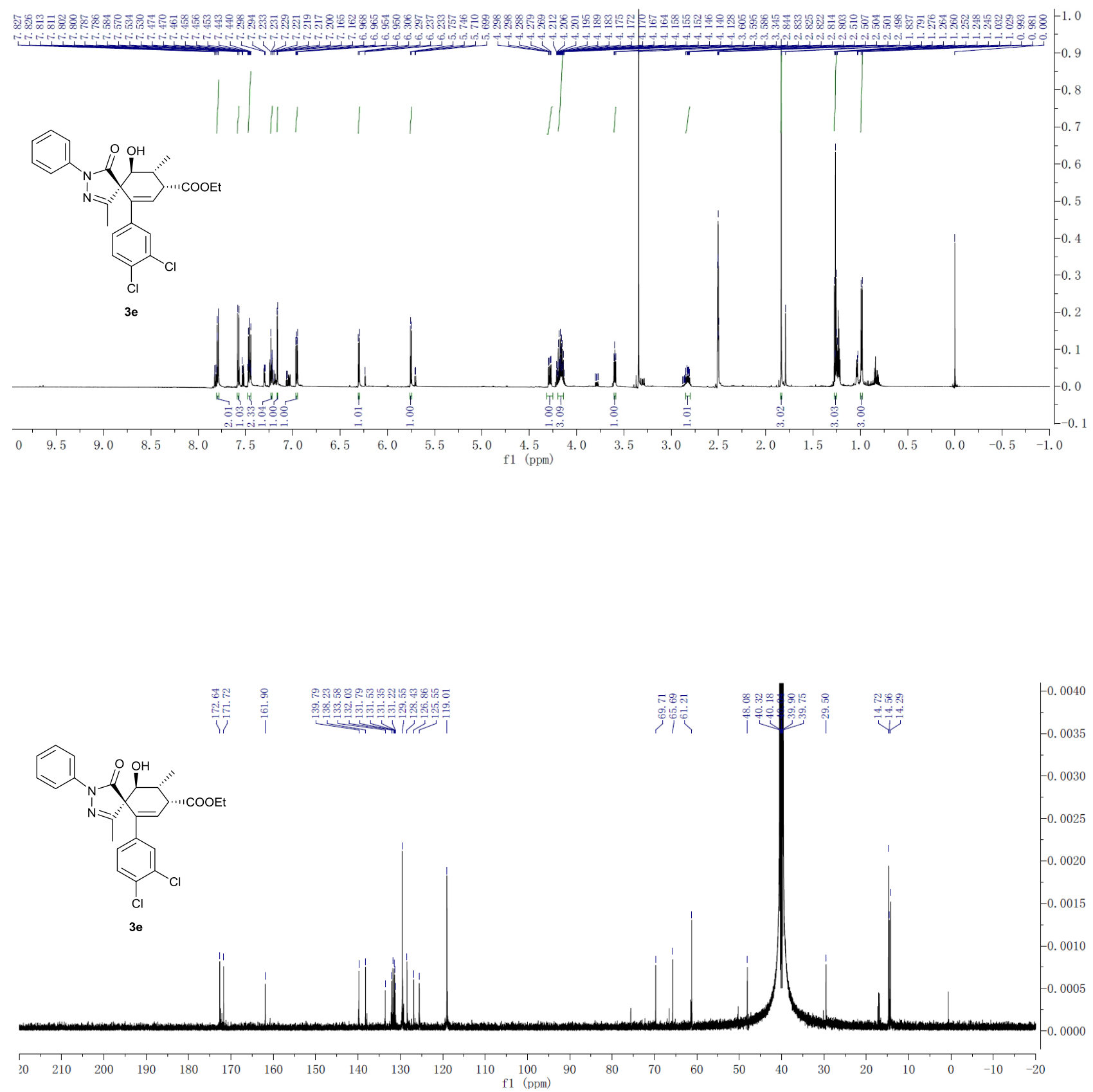

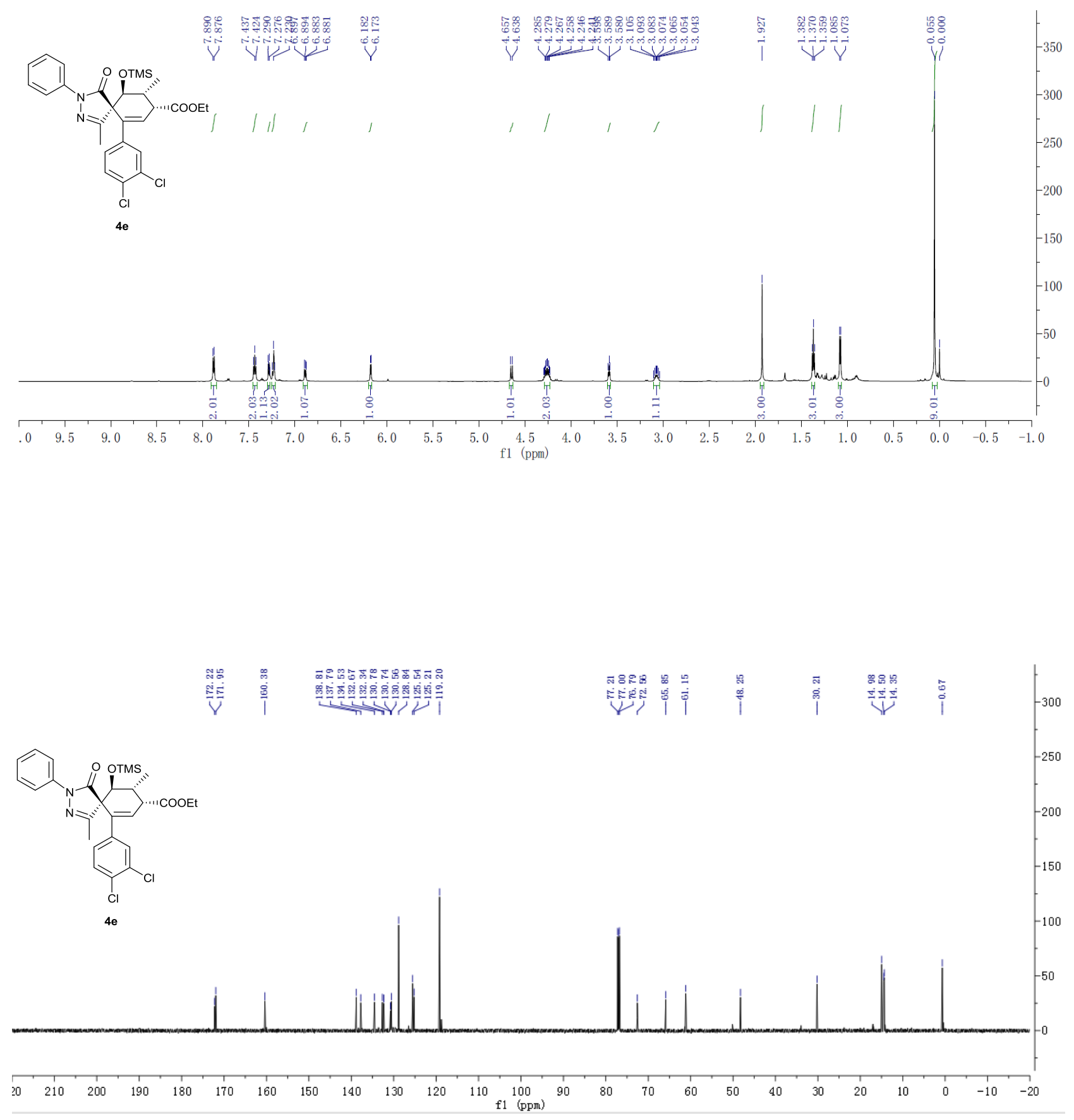
Peak Analysis Report

Detector A Channel 2 254nm

\begin{tabular}{|c|c|c|c|c|}
\hline No. & Ret. Time & Height $(\mathrm{mAu})$ & Area $\left(\mathrm{mAu}^{*} \mathrm{~min}\right)$ & Rel. Area $(\%)$ \\
\hline 1 & 5.028 & 142930 & 1316670 & 50.298 \\
\hline 2 & 8.424 & 83373 & 1301045 & 49.702 \\
\hline Total & & 226303 & 2617715 & 100.000 \\
\hline
\end{tabular}

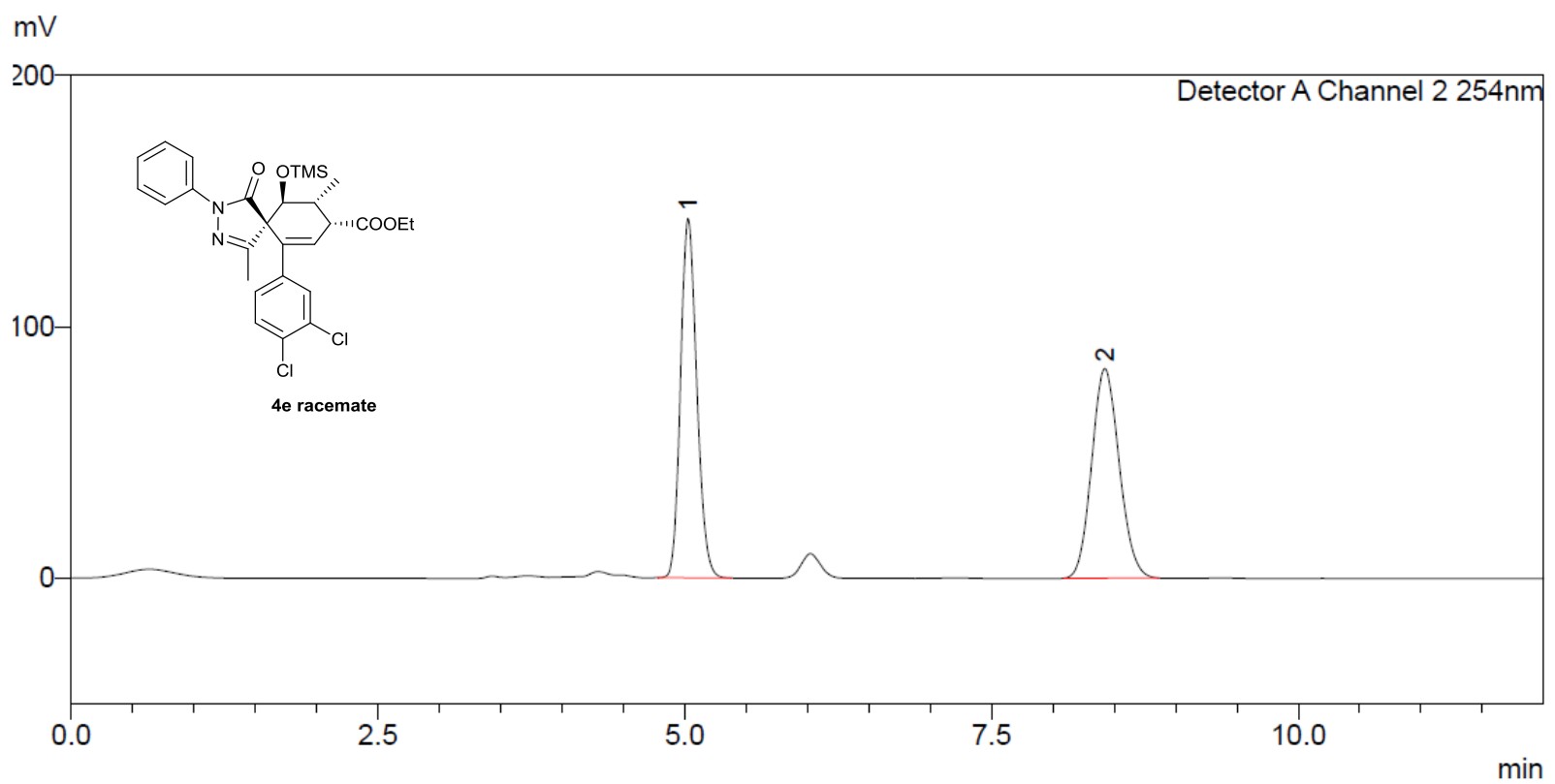

\section{Peak Analysis Report}

Detector A Channel 2 254nm

\begin{tabular}{|c|c|c|c|c|}
\hline No. & Ret. Time & Height $(\mathrm{mAu})$ & Area $\left(\mathrm{mAu}^{*} \mathrm{~min}\right)$ & Rel. Area $(\%)$ \\
\hline 1 & 4.849 & 22149 & 222490 & 3.399 \\
\hline 2 & 8.217 & 400135 & 6323732 & 96.601 \\
\hline Total & & 422284 & 6546222 & 100.000 \\
\hline
\end{tabular}

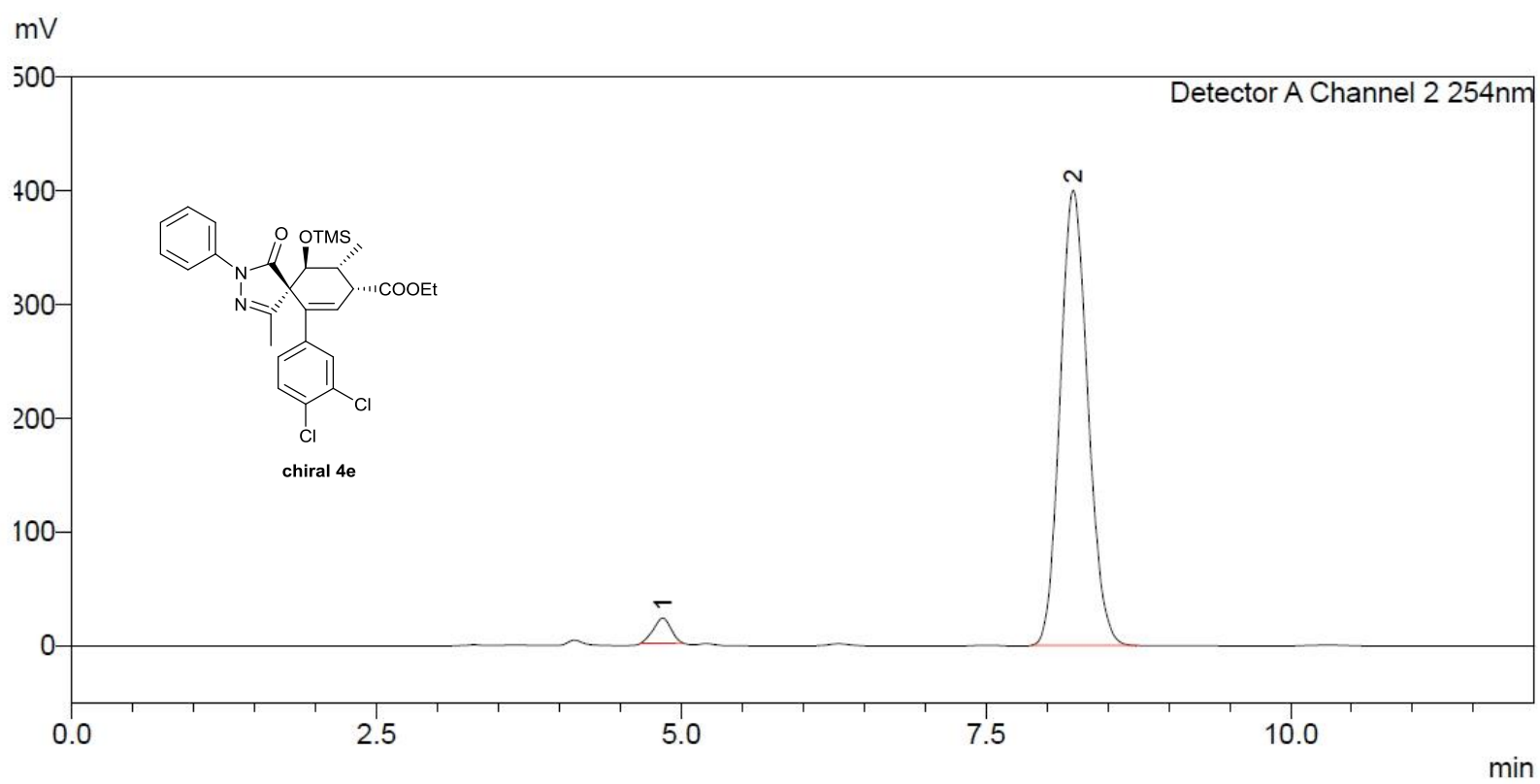



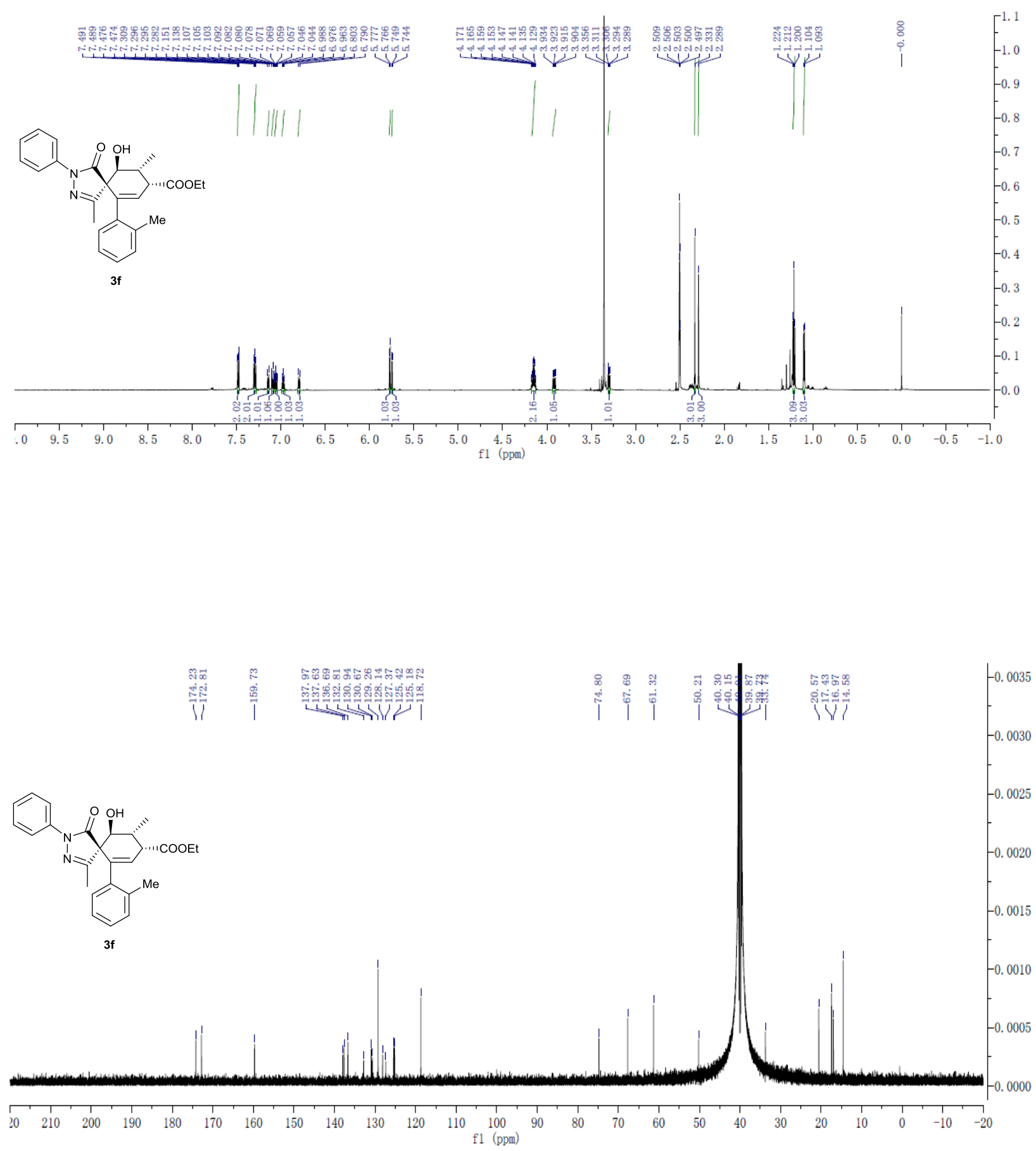

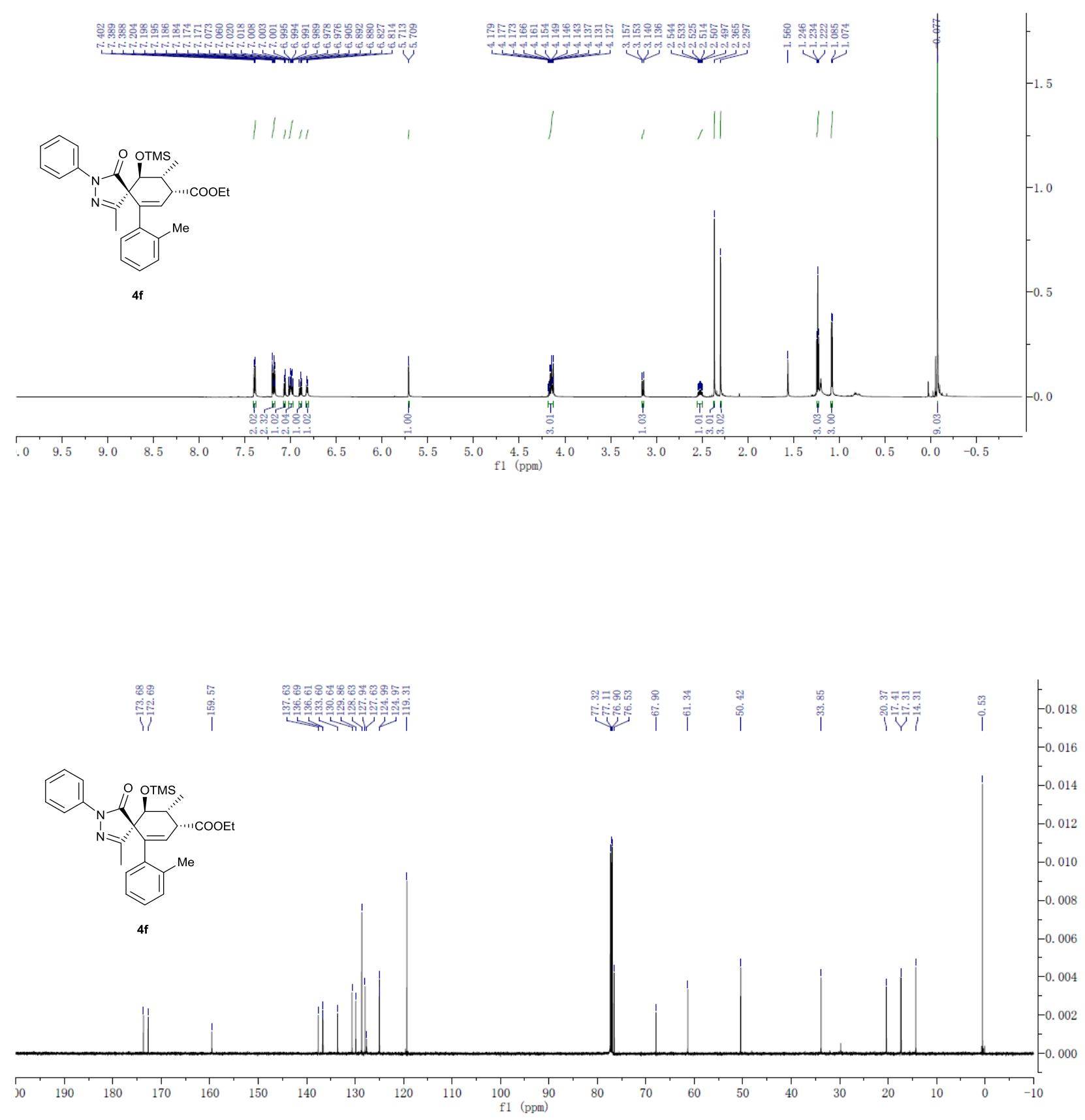


\section{Peak Analysis Report}

Detector A Channel $2254 \mathrm{~nm}$

\begin{tabular}{|c|c|c|c|c|}
\hline No. & Ret. Time & Height $(\mathrm{mAu})$ & Area $\left(\mathrm{mAu}^{*} \mathrm{~min}\right)$ & Rel. Area (\%) \\
\hline 1 & 4.286 & 228493 & 2398705 & 50.014 \\
\hline 2 & 4.751 & 202427 & 2397380 & 49.986 \\
\hline Total & & 430920 & 4796086 & 100.000 \\
\hline
\end{tabular}

$\mathrm{mV}$

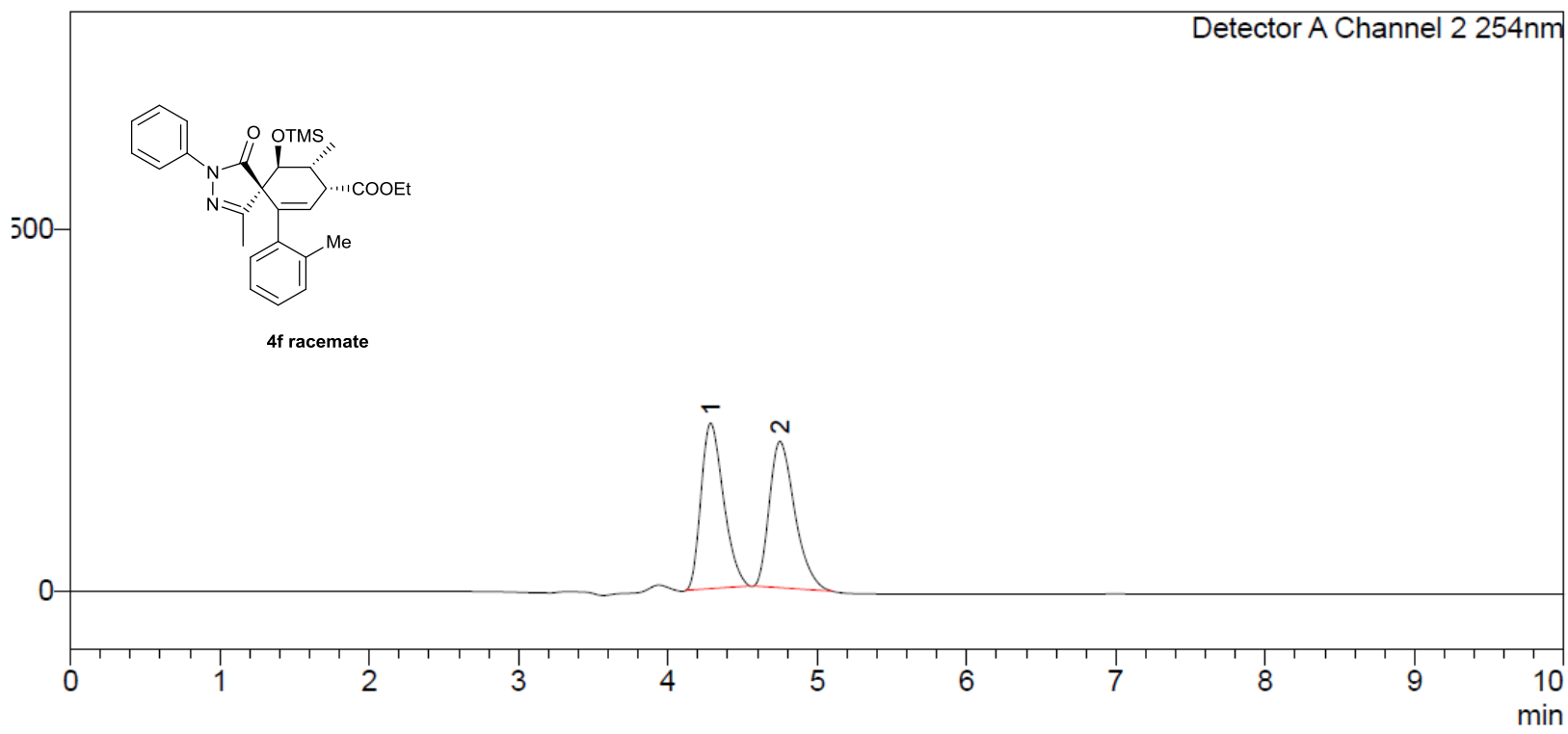

\section{Peak Analysis Report}

Detector A Channel $2254 \mathrm{~nm}$

\begin{tabular}{|c|c|c|c|c|}
\hline No. & Ret. Time & Height $(\mathrm{mAu})$ & Area $\left(\mathrm{mAu}^{*} \mathrm{~min}\right)$ & Rel. Area $(\%)$ \\
\hline 1 & 4.188 & 28581 & 238881 & 5.003 \\
\hline 2 & 4.610 & 466693 & 4536091 & 94.997 \\
\hline Total & & 495273 & 4774972 & 100.000 \\
\hline
\end{tabular}

$\mathrm{mV}$

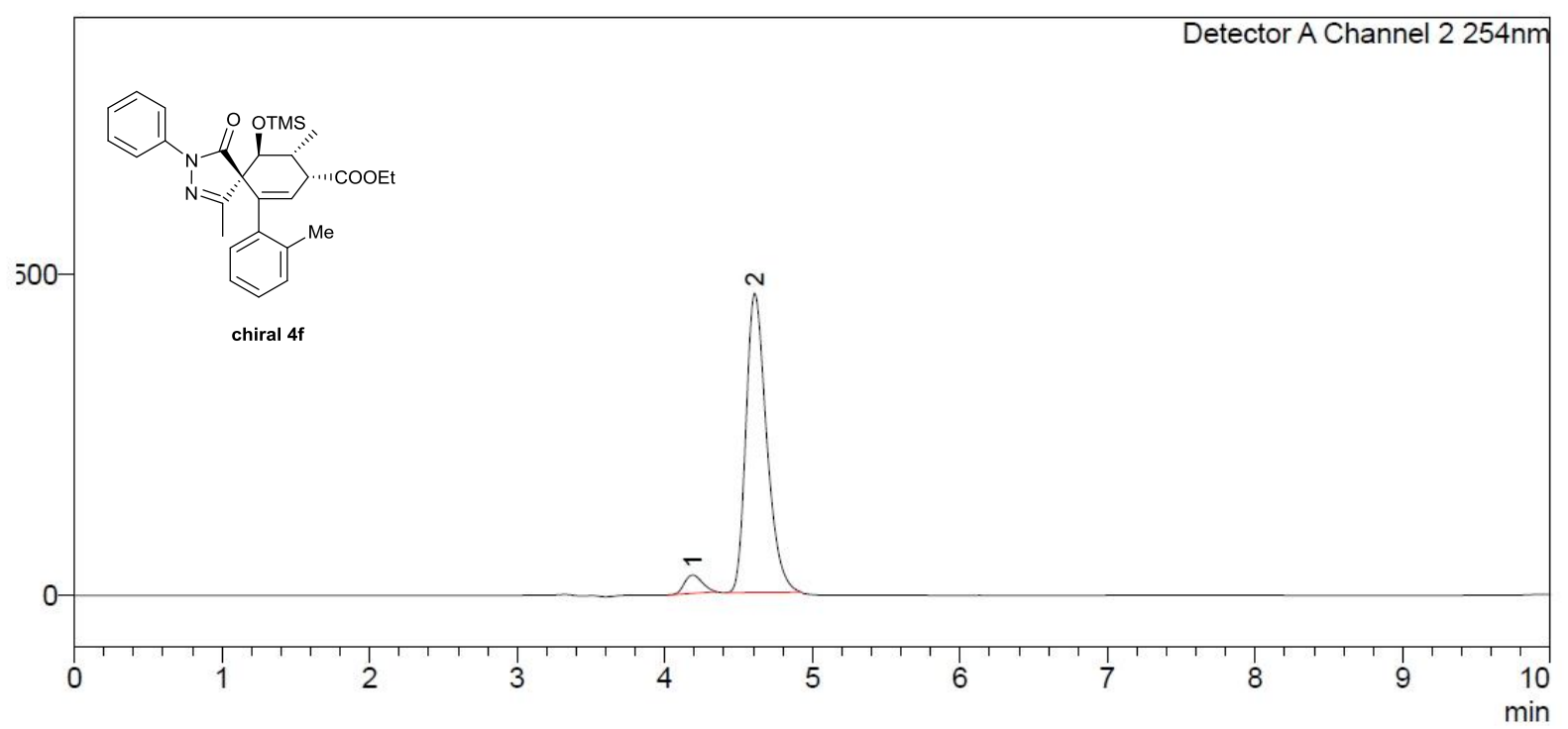



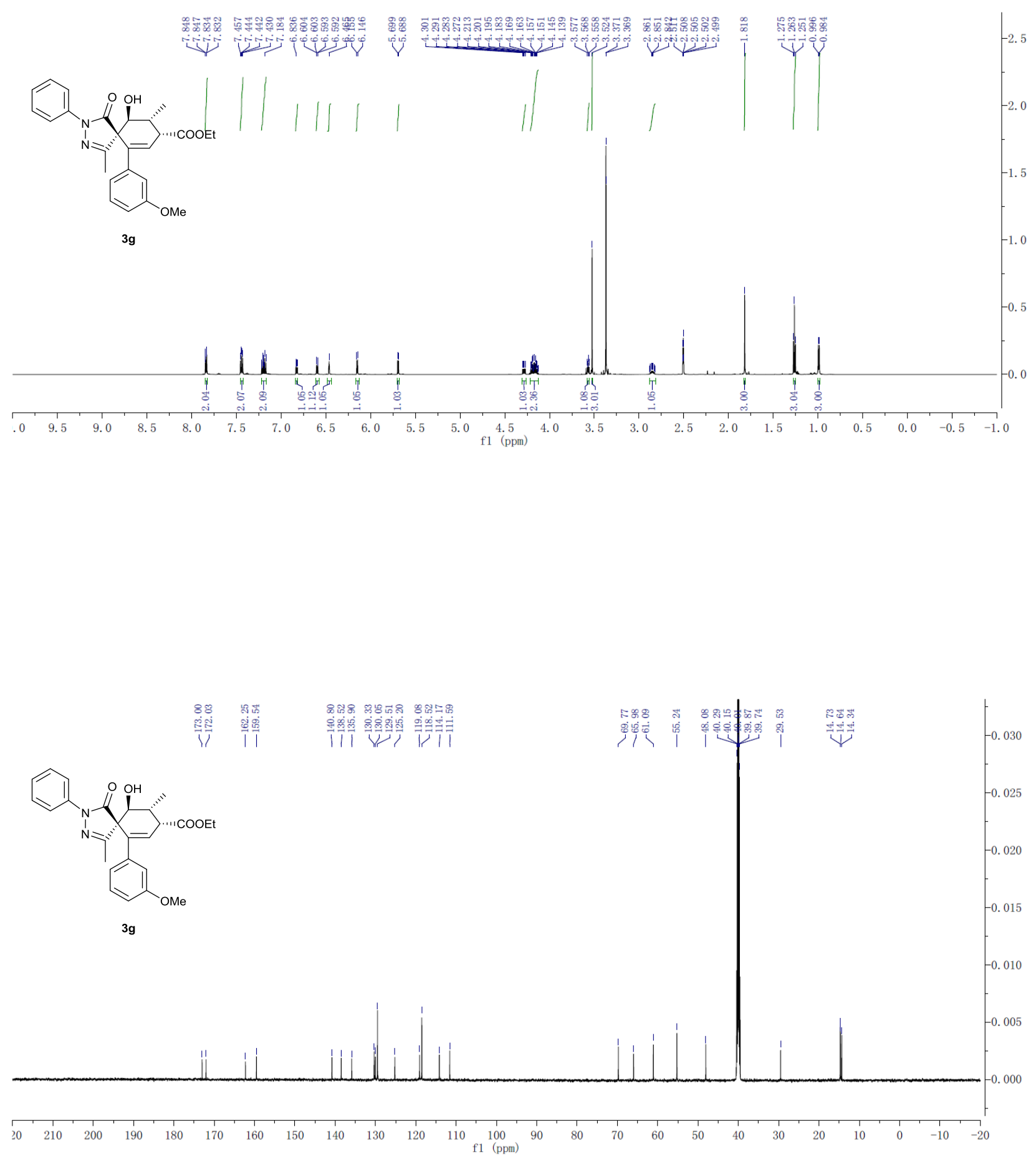

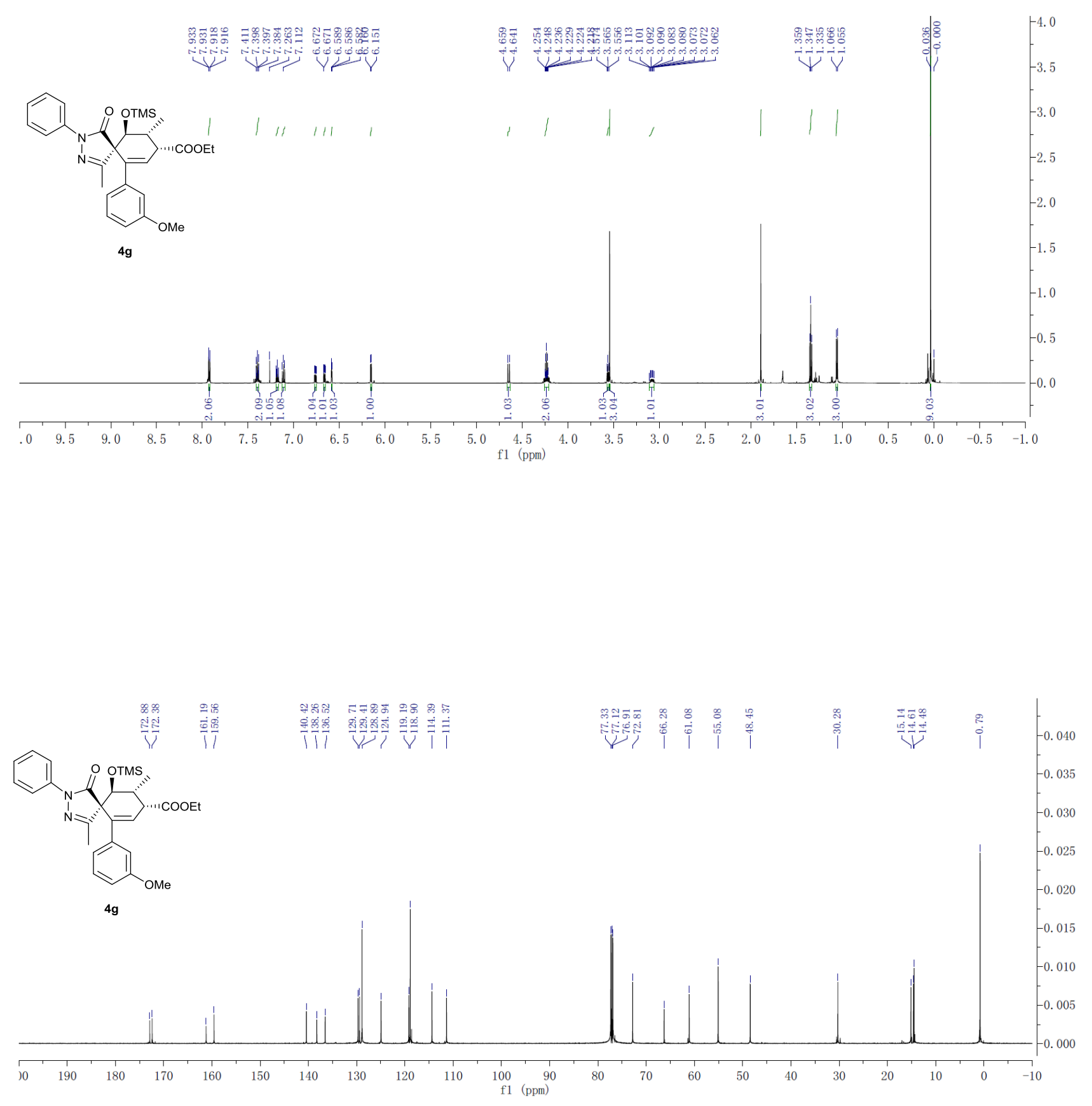

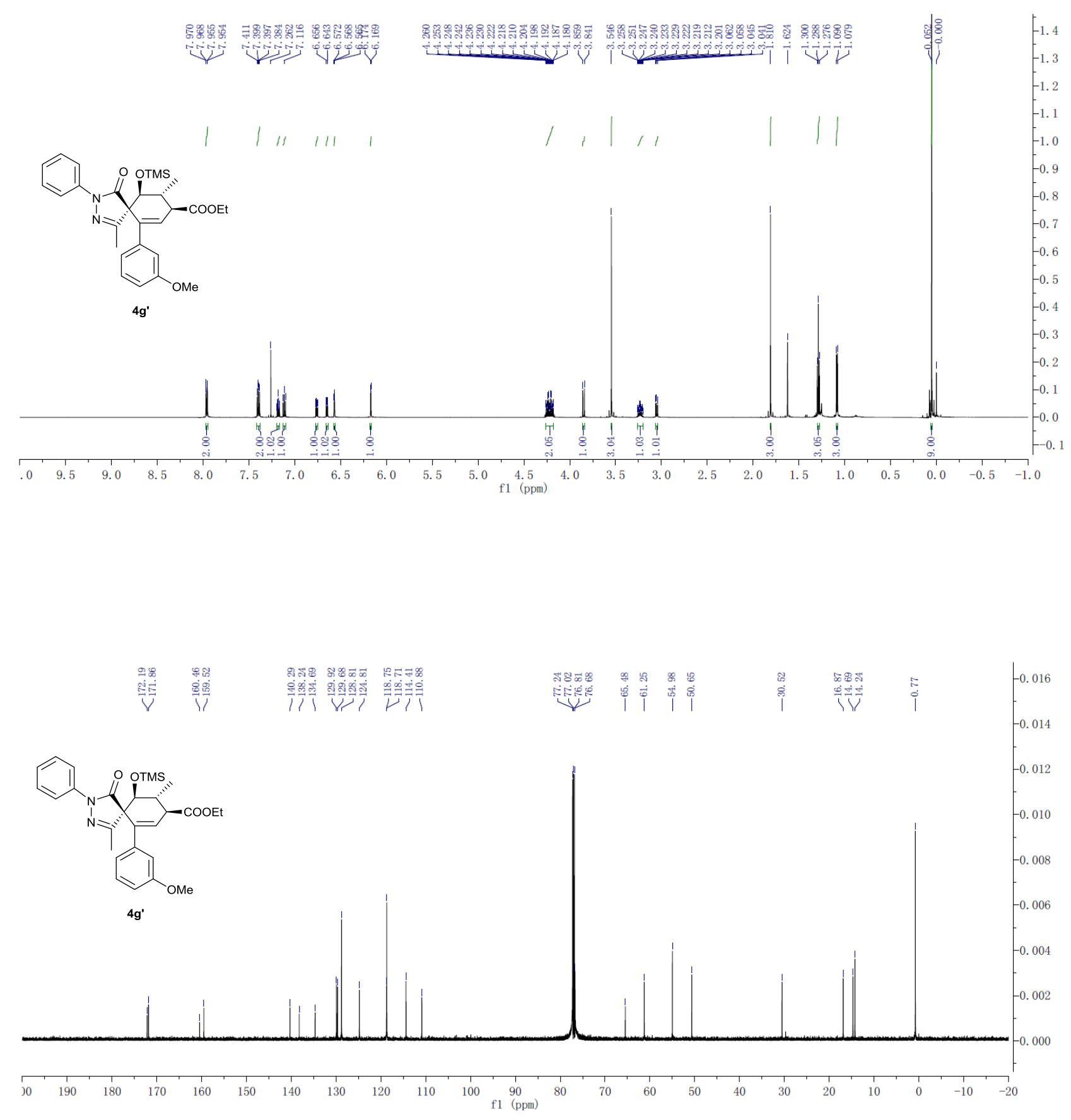


\section{Peak Analysis Report}

Detector A Channel $2254 \mathrm{~nm}$

\begin{tabular}{|c|c|c|c|c|}
\hline No. & Ret. Time & Height (mAu) & Area (mAu*min) & Rel. Area (\%) \\
\hline 1 & 4.911 & 415317 & 4244522 & 49.970 \\
\hline 2 & 5.432 & 376813 & 4249558 & 50.030 \\
\hline Total & & 792130 & 8494080 & 100.000 \\
\hline
\end{tabular}

$\mathrm{mV}$

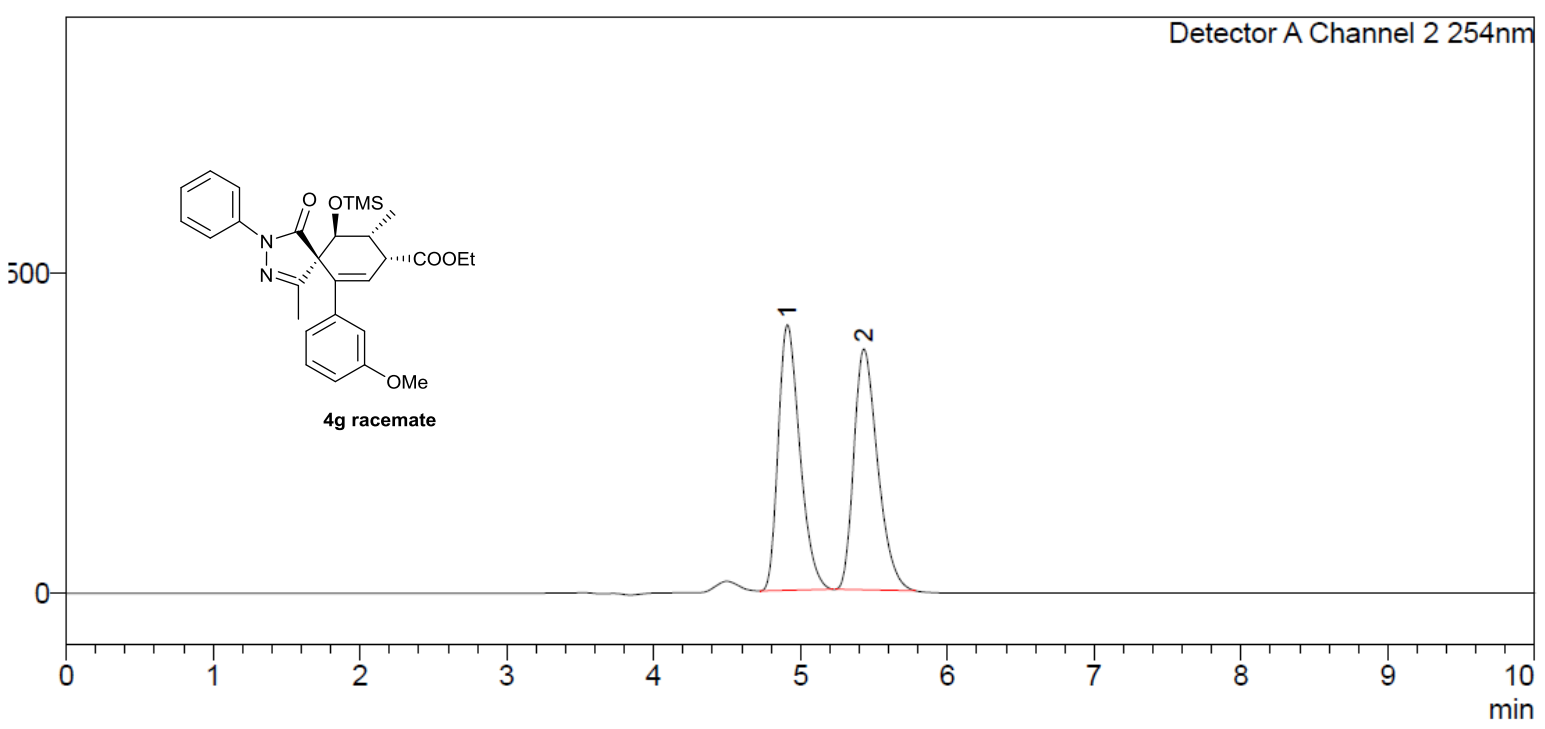

\section{Peak Analysis Report}

Detector A Channel 2 254nm

\begin{tabular}{|c|c|c|c|c|}
\hline No. & Ret. Time & Height $(\mathrm{mAu})$ & Area $\left(\mathrm{mAu}{ }^{*} \mathrm{~min}\right)$ & Rel. Area $(\%)$ \\
\hline 1 & 5.109 & 84941 & 864336 & 4.995 \\
\hline 2 & 5.594 & 1389232 & 16438144 & 95.005 \\
\hline Total & & 1474172 & 17302480 & 100.000 \\
\hline
\end{tabular}

$\mathrm{mV}$

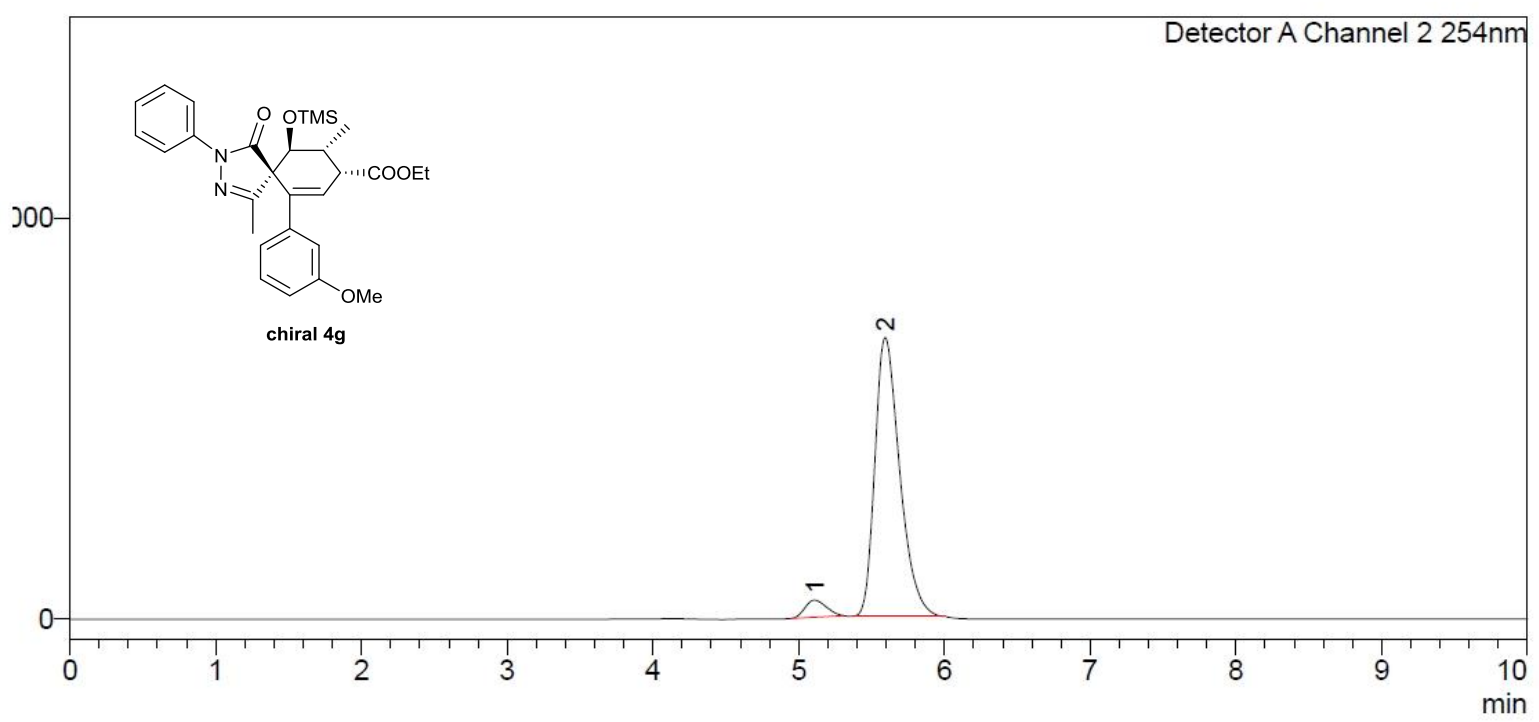




\section{Peak Analysis Report}

Detector A Channel $2254 \mathrm{~nm}$
\begin{tabular}{|c|c|c|c|c|}
\hline No. & Ret. Time & Height (mAu) & Area (mAu*min) & Rel. Area (\%) \\
\hline 1 & 24.542 & 162009 & 6763921 & 50.822 \\
\hline 2 & 31.560 & 120818 & 6545108 & 49.178 \\
\hline Total & & 282827 & 13309029 & 100.000 \\
\hline
\end{tabular}

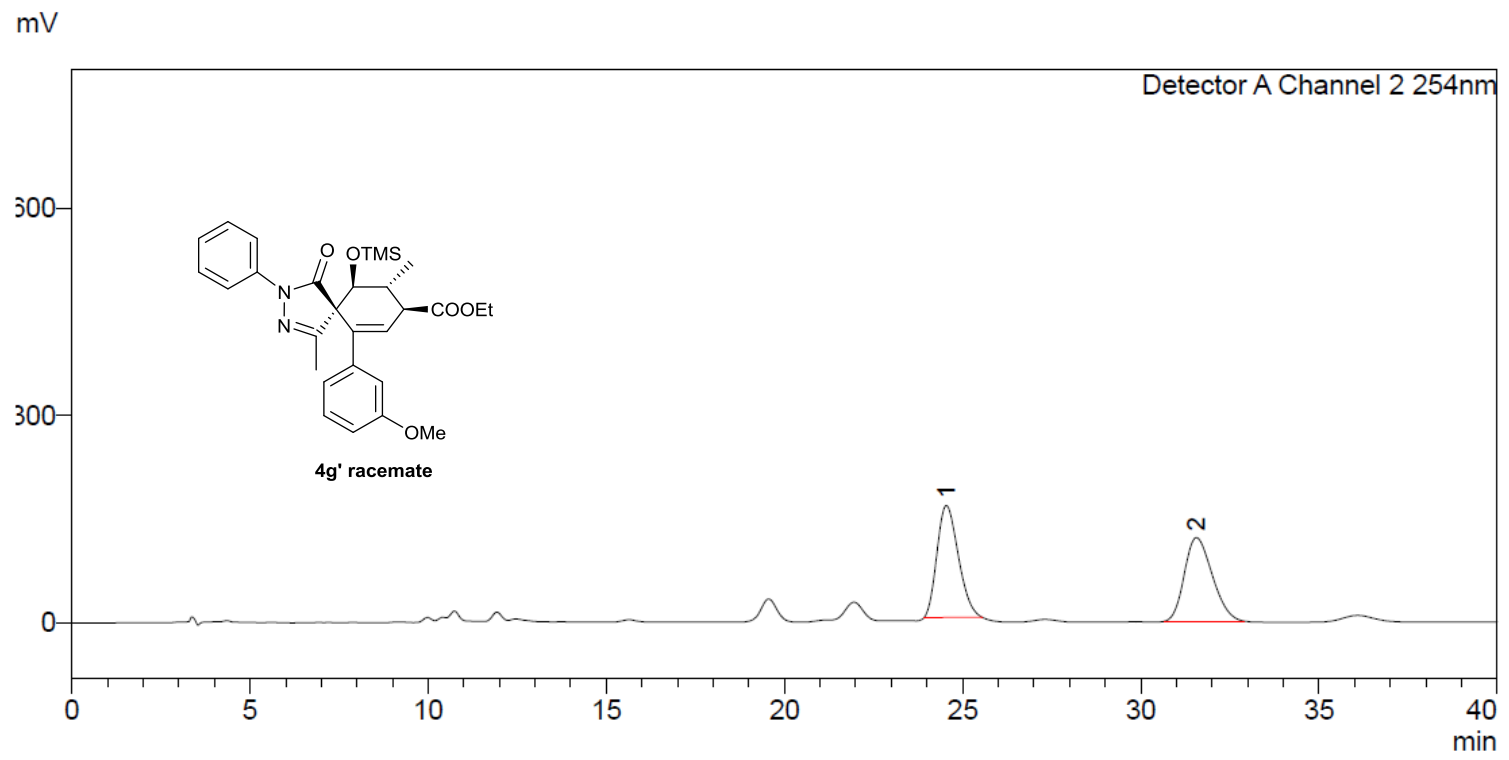

\section{Peak Analysis Report}

Detector A Channel 2 254nm

\begin{tabular}{|c|c|c|c|c|}
\hline No. & Ret. Time & Height $(\mathrm{mAu})$ & Area $\left(\mathrm{mAu}^{\star} \mathrm{min}\right)$ & Rel. Area (\%) \\
\hline 1 & 24.530 & 77693 & 3016918 & 30.290 \\
\hline 2 & 31.519 & 126322 & 6943252 & 69.710 \\
\hline Total & & 204015 & 9960170 & 100.000 \\
\hline
\end{tabular}

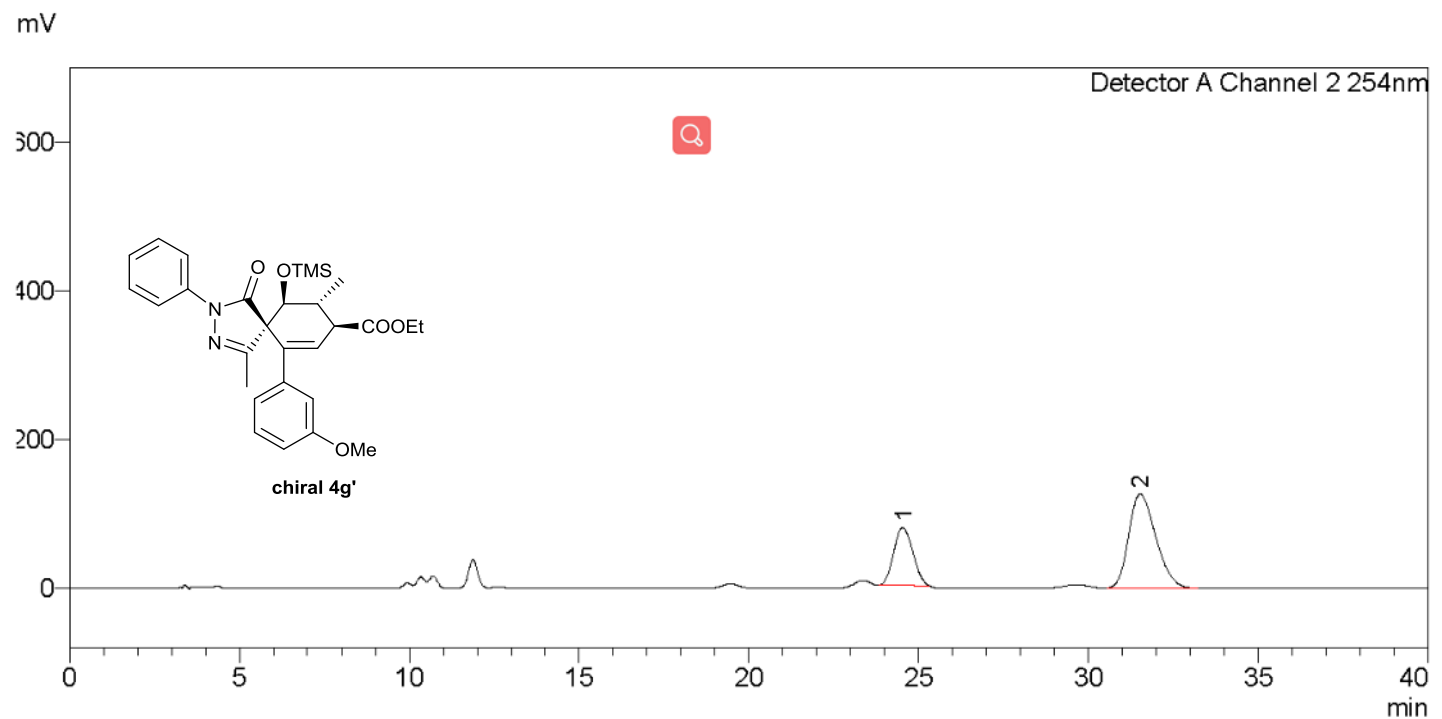



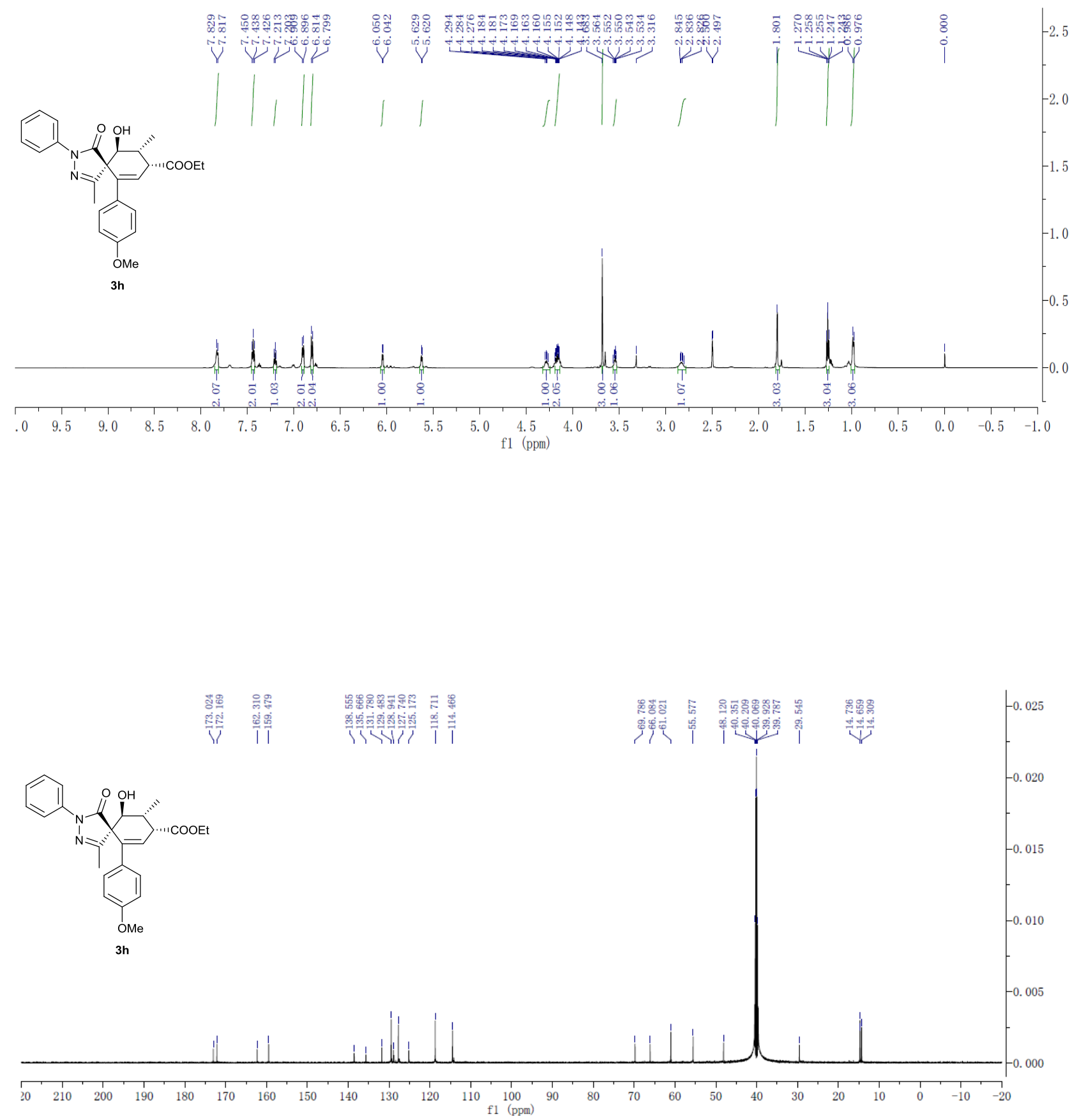


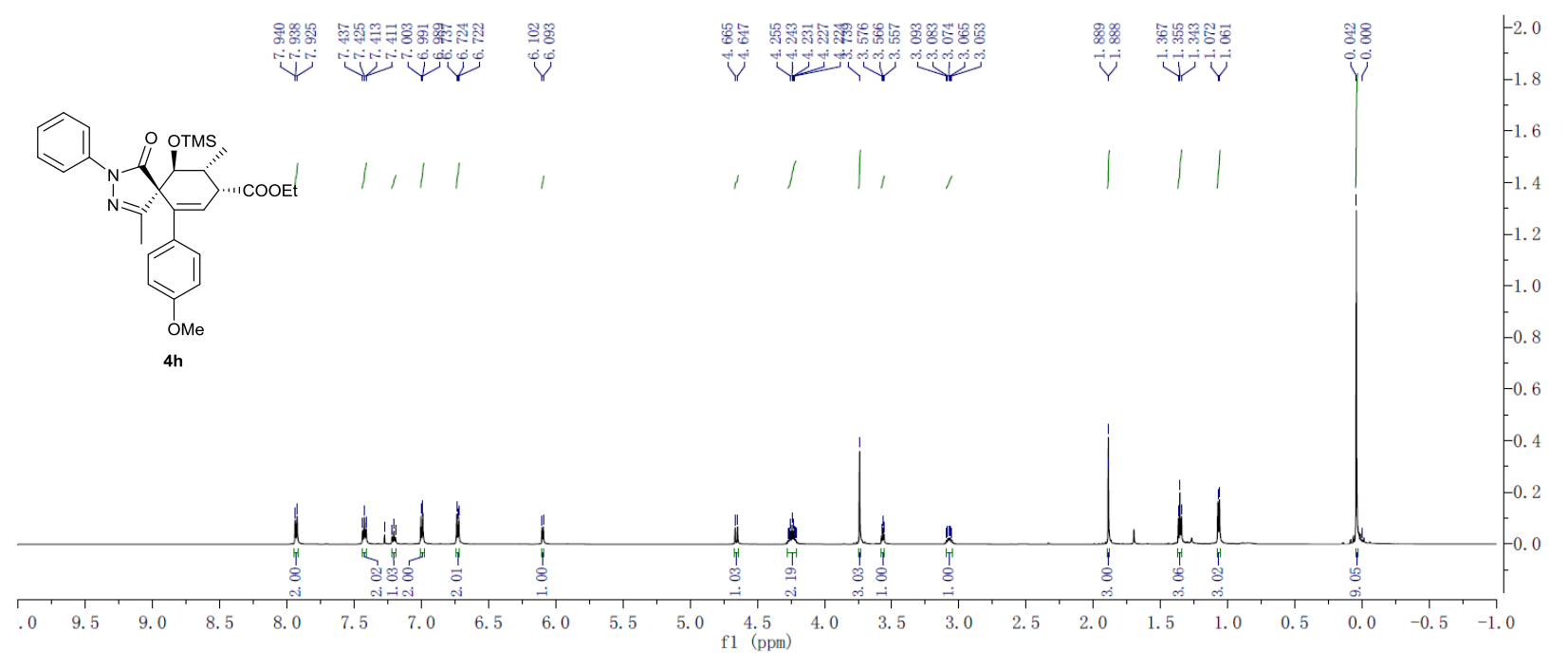

Z

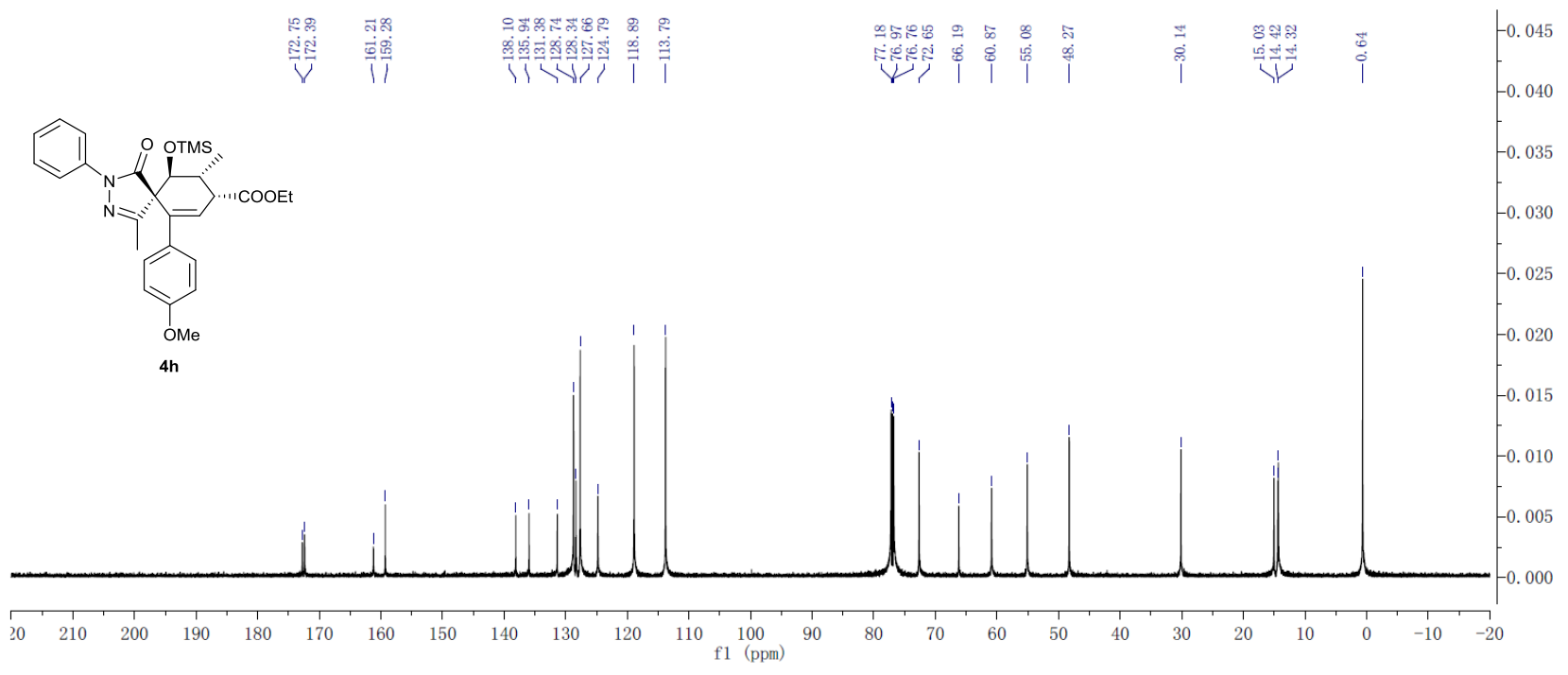



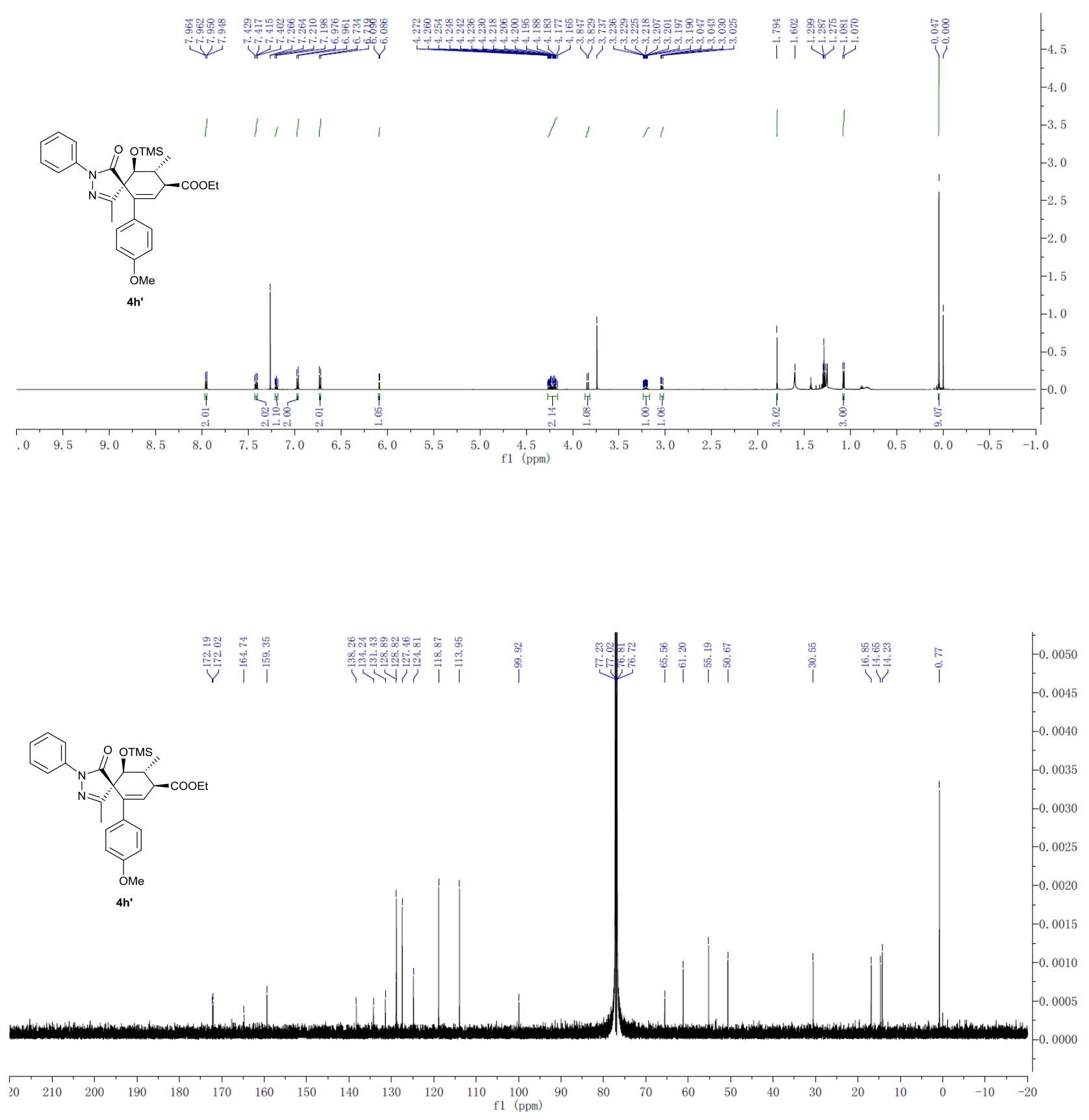


\section{Peak Analysis Report}

Detector A Channel $2254 \mathrm{~nm}$

\begin{tabular}{|c|c|c|c|c|}
\hline No. & Ret. Time & Height $(\mathrm{mAu})$ & Area $\left(\mathrm{mAu}^{*} \mathrm{~min}\right)$ & Rel. Area $(\%)$ \\
\hline 1 & 13.540 & 70167 & 1763772 & 50.442 \\
\hline 2 & 15.662 & 51559 & 1732891 & 49.558 \\
\hline Total & & 121725 & 3496662 & 100.000 \\
\hline
\end{tabular}

$\mathrm{mV}$

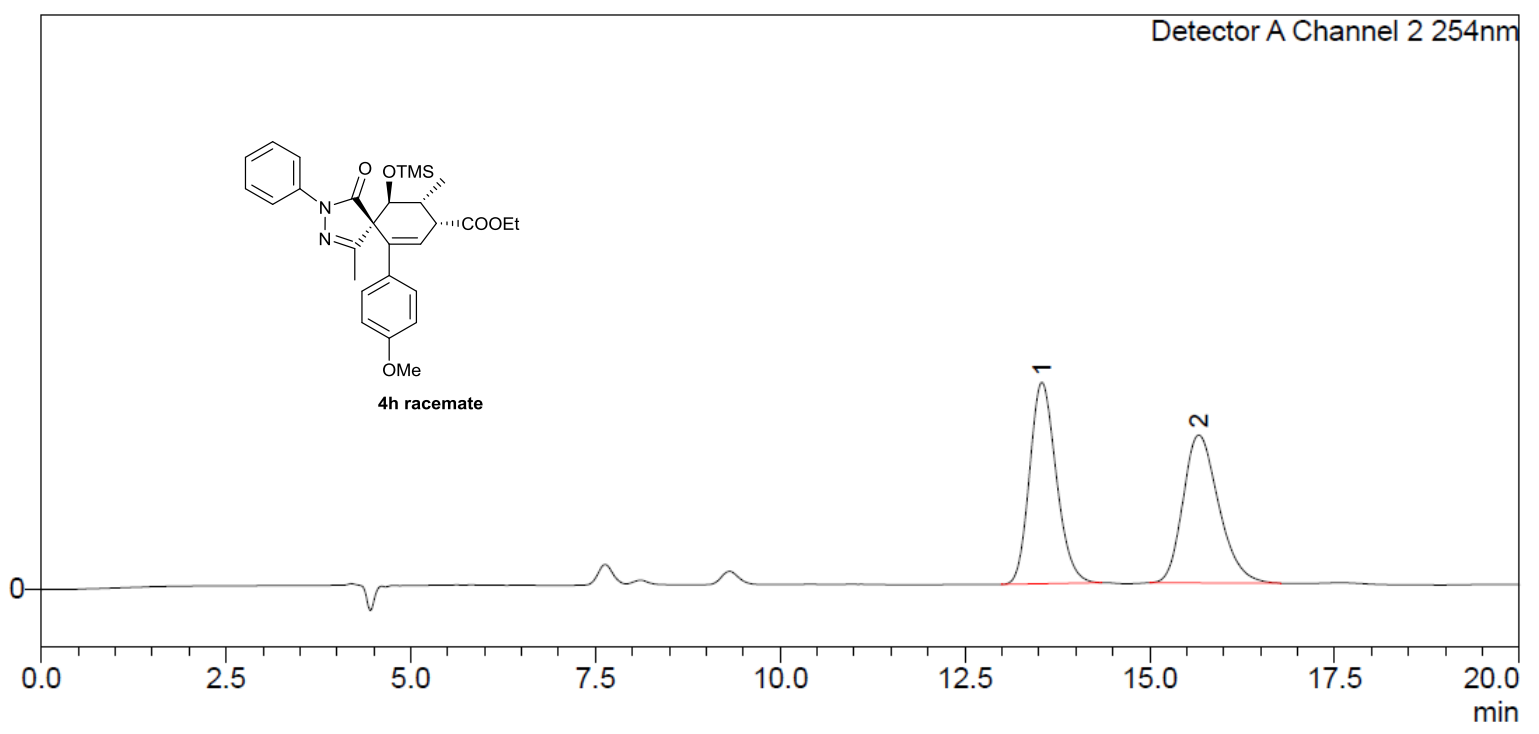

\section{Peak Analysis Report}

Detector A Channel $2254 \mathrm{~nm}$

\begin{tabular}{|c|c|c|c|c|}
\hline No. & Ret. Time & Height $(\mathrm{mAu})$ & Area $\left(\mathrm{mAu}^{*} \mathrm{~min}\right)$ & Rel. Area $(\%)$ \\
\hline 1 & 13.335 & 2806 & 56971 & 0.822 \\
\hline 2 & 15.856 & 197172 & 6869698 & 99.178 \\
\hline Total & & 199978 & 6926669 & 100.000 \\
\hline
\end{tabular}

$\mathrm{mV}$

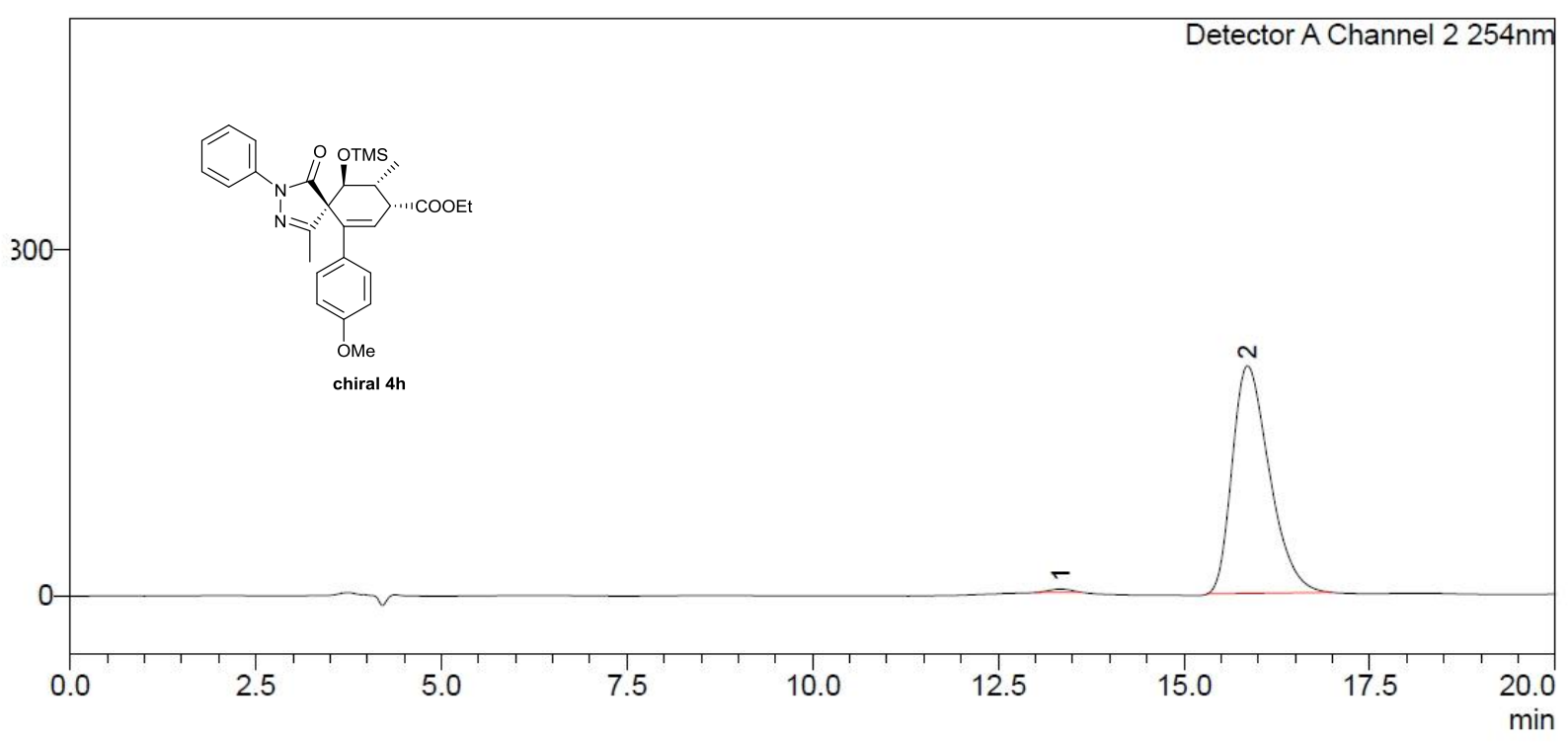




\section{Peak Analysis Report}

Detector A Channel $2254 \mathrm{~nm}$

\begin{tabular}{|c|c|c|c|c|}
\hline No. & Ret. Time & Height $(\mathrm{mAu})$ & Area $\left(\mathrm{mAu}^{*} \mathrm{~min}\right)$ & Rel. Area $(\%)$ \\
\hline 1 & 11.299 & 545143 & 12026203 & 50.239 \\
\hline 2 & 16.667 & 336309 & 11911989 & 49.761 \\
\hline Total & & 881452 & 23938192 & 100.000 \\
\hline
\end{tabular}

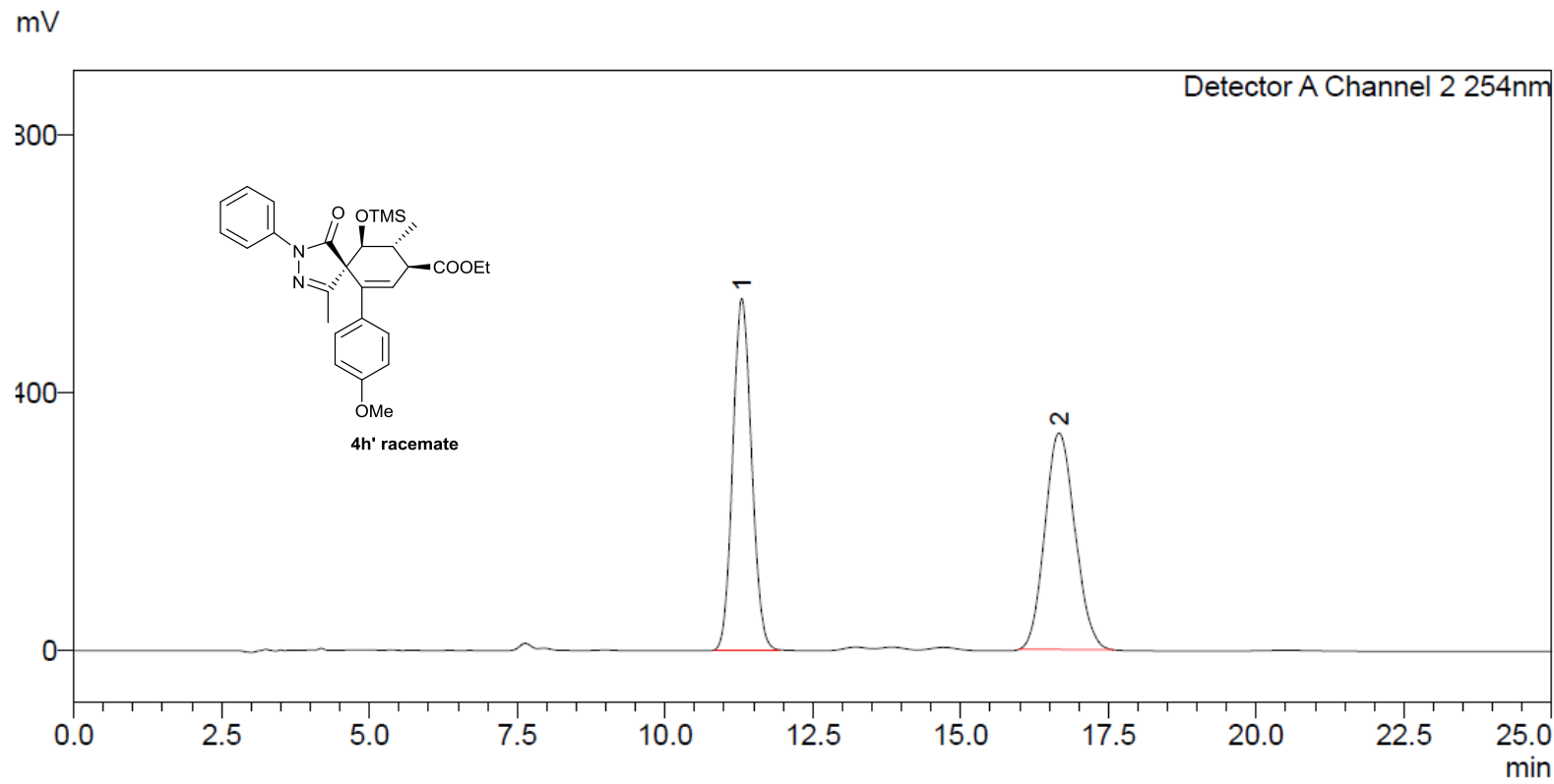

\section{Peak Analysis Report}

Detector A Channel $2254 \mathrm{~nm}$

\begin{tabular}{|c|c|c|c|c|}
\hline No. & Ret. Time & Height $(\mathrm{mAu})$ & Area $\left(\mathrm{mAu}^{*} \mathrm{~min}\right)$ & Rel. Area $(\%)$ \\
\hline 1 & 11.312 & 265796 & 5843447 & 43.780 \\
\hline 2 & 16.677 & 211337 & 7503835 & 56.220 \\
\hline Total & & 477133 & 13347282 & 100.000 \\
\hline
\end{tabular}

$\mathrm{mV}$

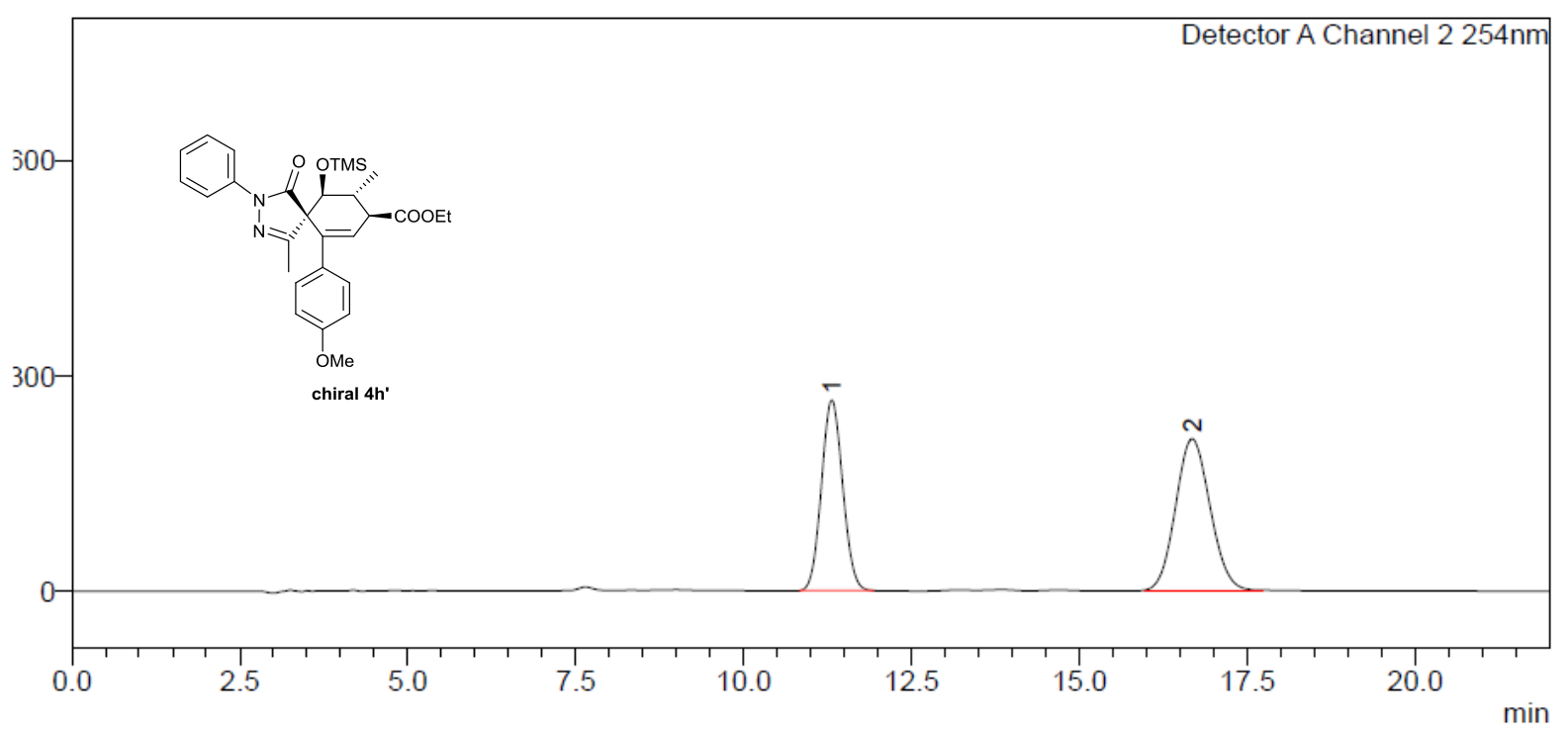



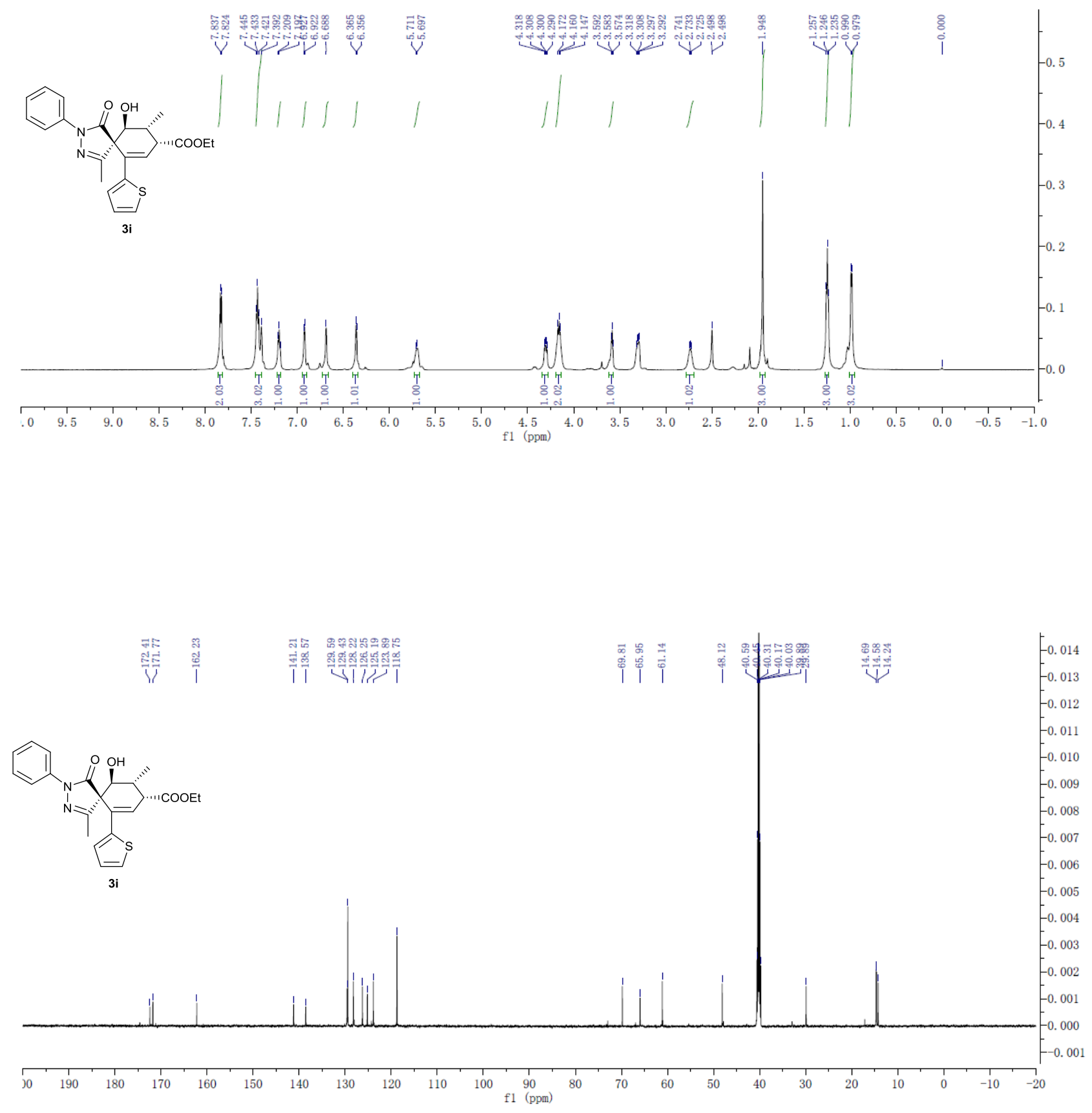

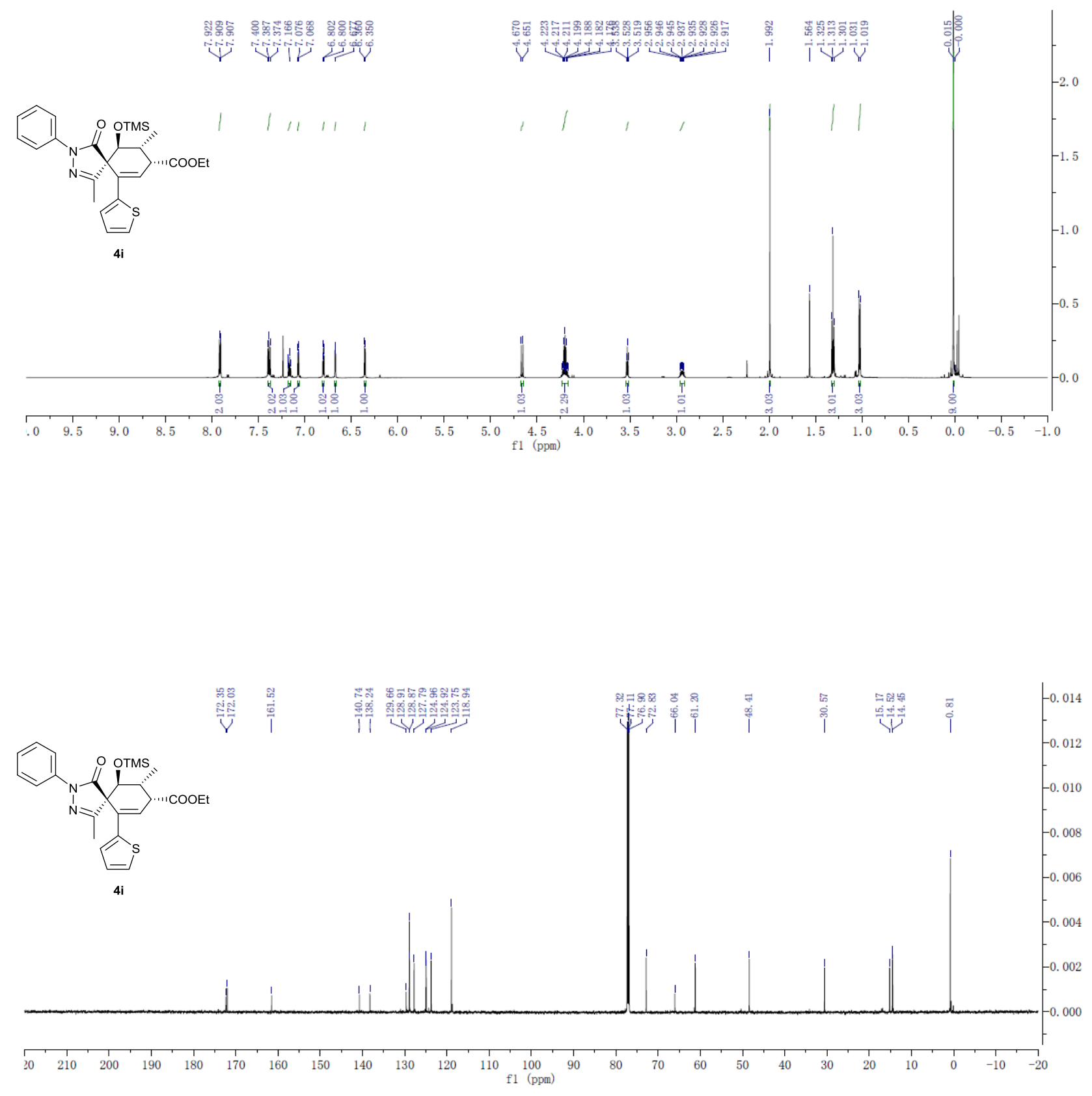


\section{Peak Analysis Report}

Detector A Channel 2 254nm

\begin{tabular}{|c|c|c|c|c|}
\hline No. & Ret. Time & Height $(\mathrm{mAu})$ & Area $\left(\mathrm{mAu}^{*} \mathrm{~min}\right)$ & Rel. Area (\%) \\
\hline 1 & 10.090 & 214501 & 3408081 & 48.067 \\
\hline 2 & 31.284 & 69681 & 3682251 & 51.933 \\
\hline Total & & 284182 & 7090332 & 100.000 \\
\hline
\end{tabular}

$\mathrm{mV}$

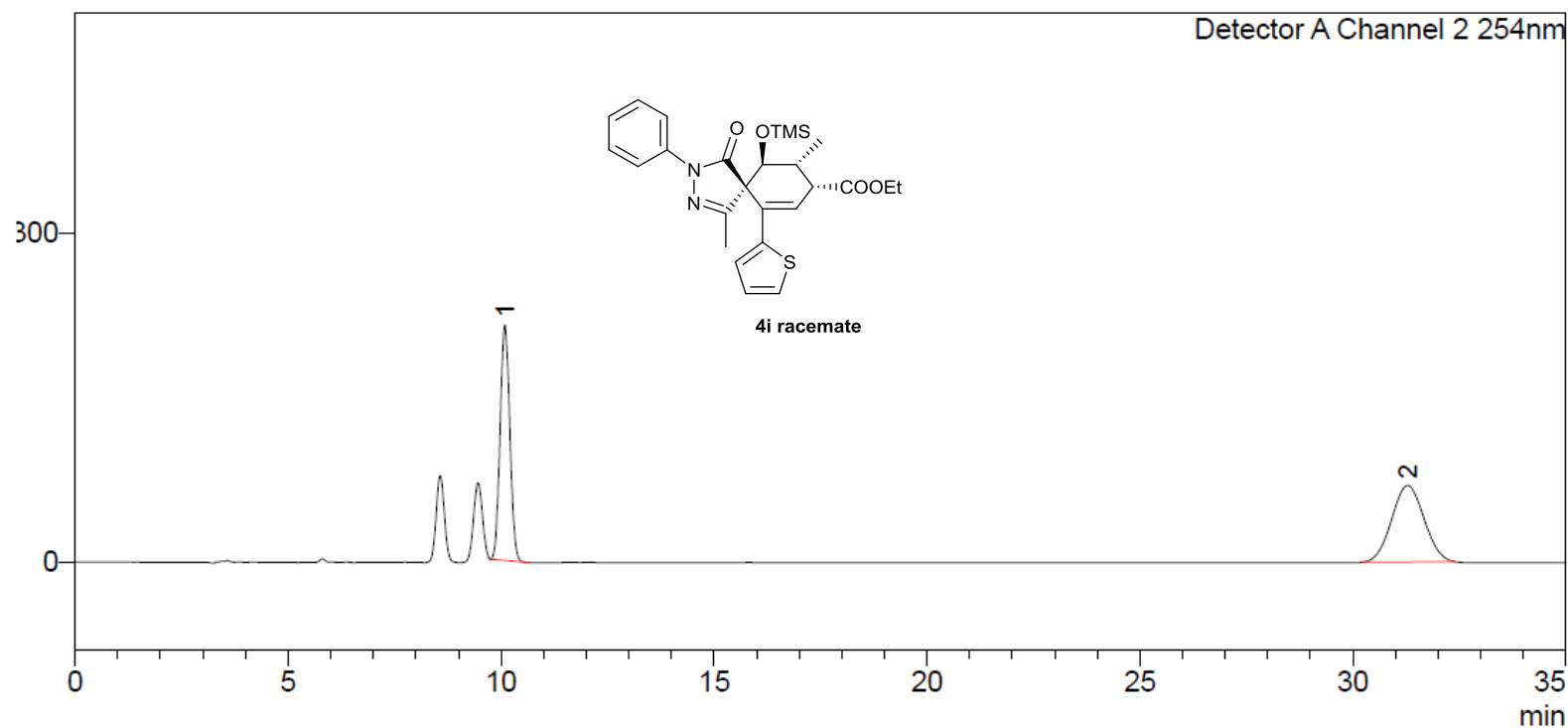

\section{Peak Analysis Report}

Detector A Channel 2 254nm

\begin{tabular}{|c|c|c|c|c|}
\hline Detector A Chann & Ret. Time & Height $(\mathrm{mAu})$ & Area $\left(\mathrm{mAu}^{*} \mathrm{~min}\right)$ & Rel. Area $(\%)$ \\
\hline 1 & 10.328 & 7698 & 113697 & 3.091 \\
\hline 2 & 31.543 & 64669 & 3565011 & 96.909 \\
\hline Total & & 72368 & 3678708 & 100.000 \\
\hline
\end{tabular}

$\mathrm{mV}$

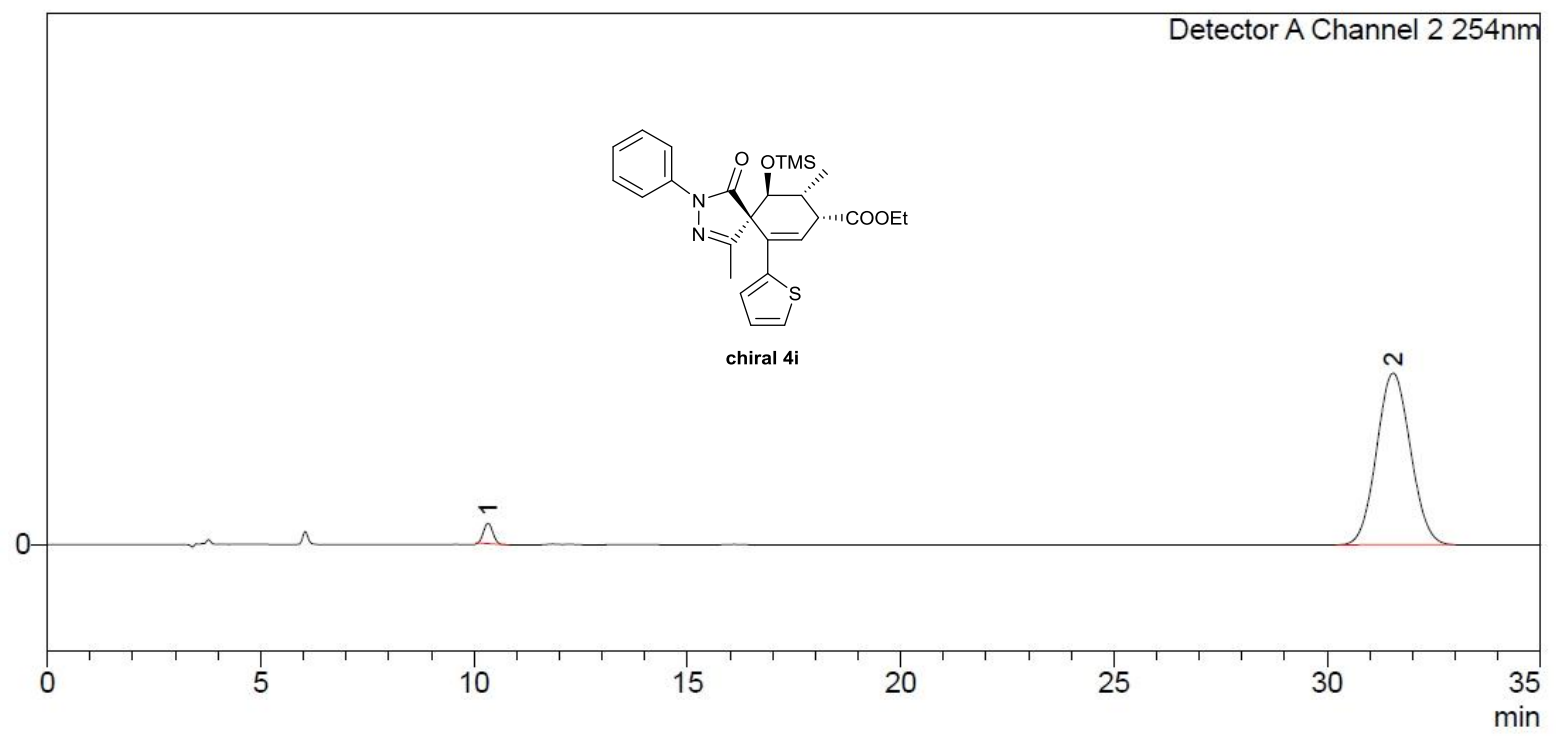



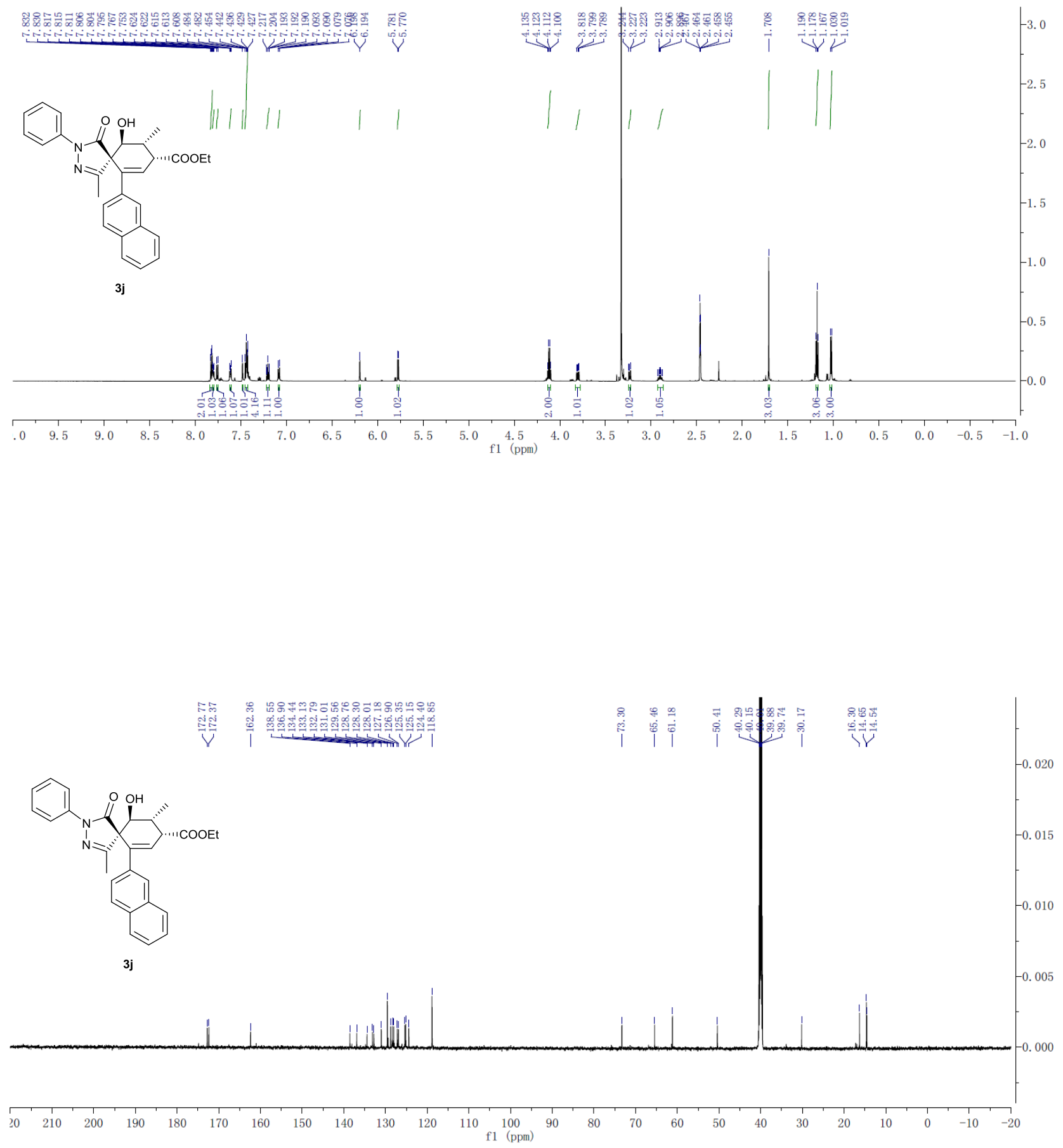

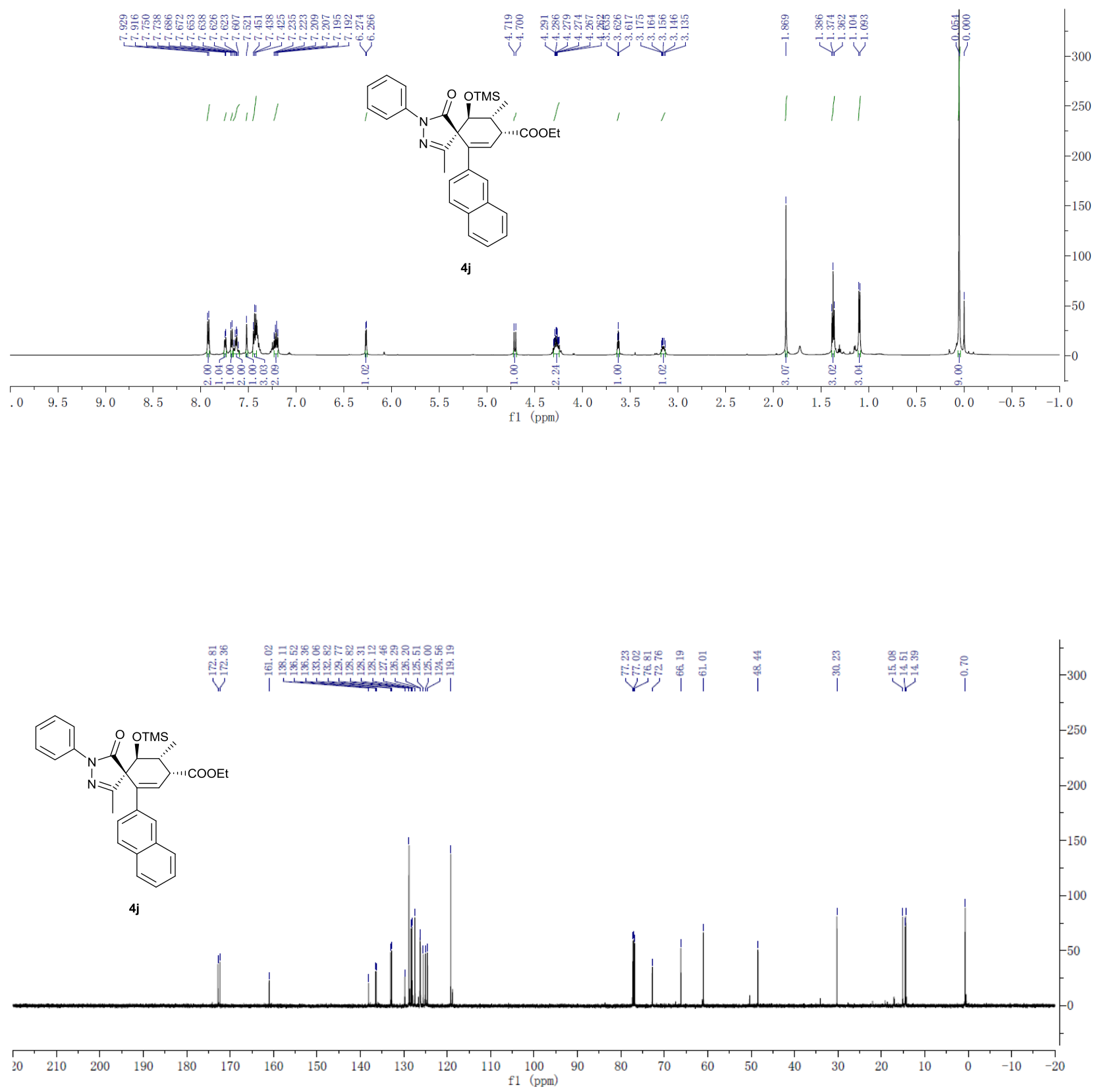


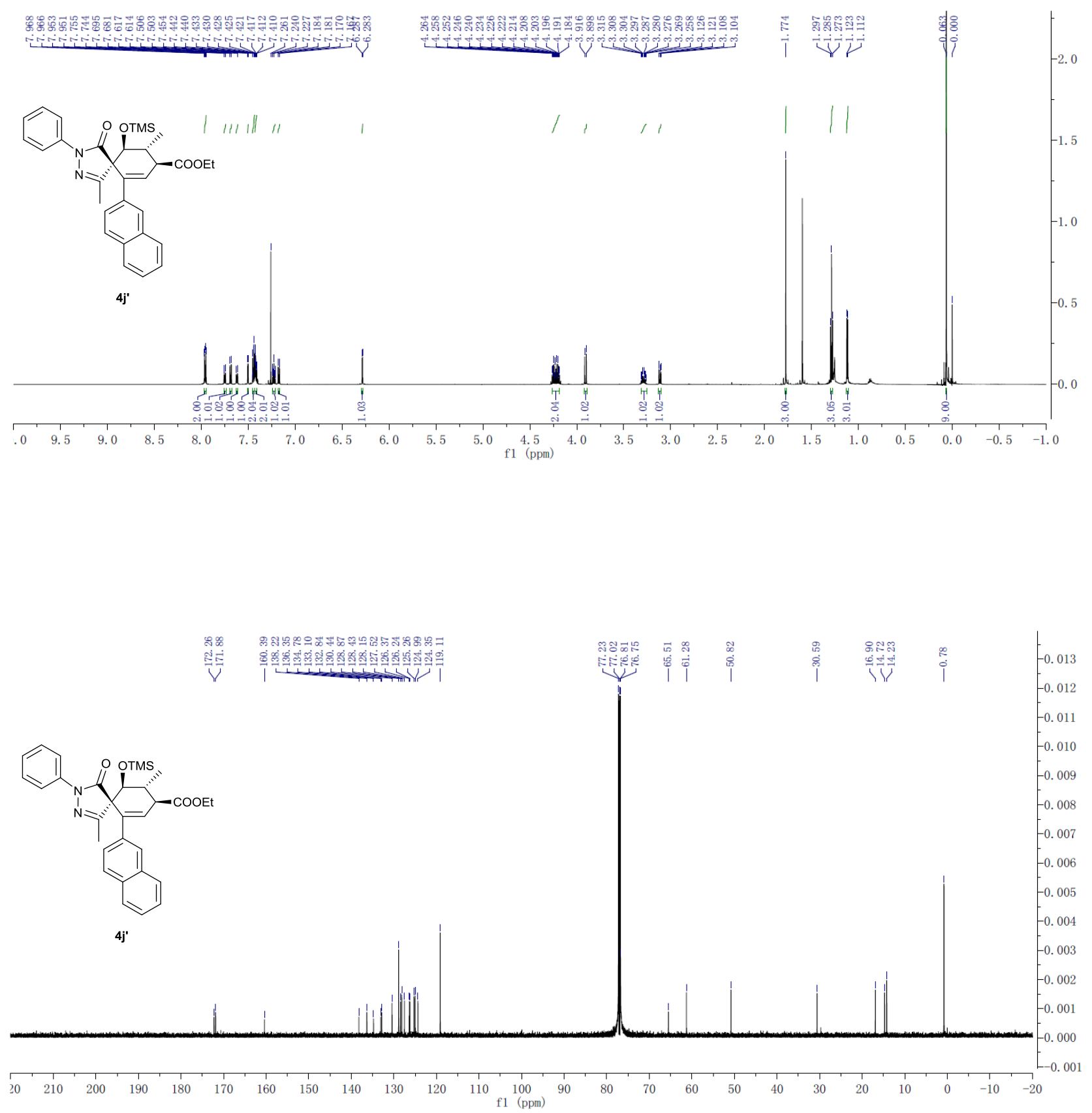




\section{Peak Analysis Report}

Detector A Channel $2254 \mathrm{~nm}$

\begin{tabular}{|c|c|c|c|c|}
\hline No. & Ret. Time & Height $(\mathrm{mAu})$ & Area $\left(\mathrm{mAu}^{*} \mathrm{~min}\right)$ & Rel. Area (\%) \\
\hline 1 & 6.531 & 104174 & 1245797 & 50.450 \\
\hline 2 & 9.312 & 54595 & 1223566 & 49.550 \\
\hline Total & & 158770 & 2469363 & 100.000 \\
\hline
\end{tabular}

$\mathrm{mV}$

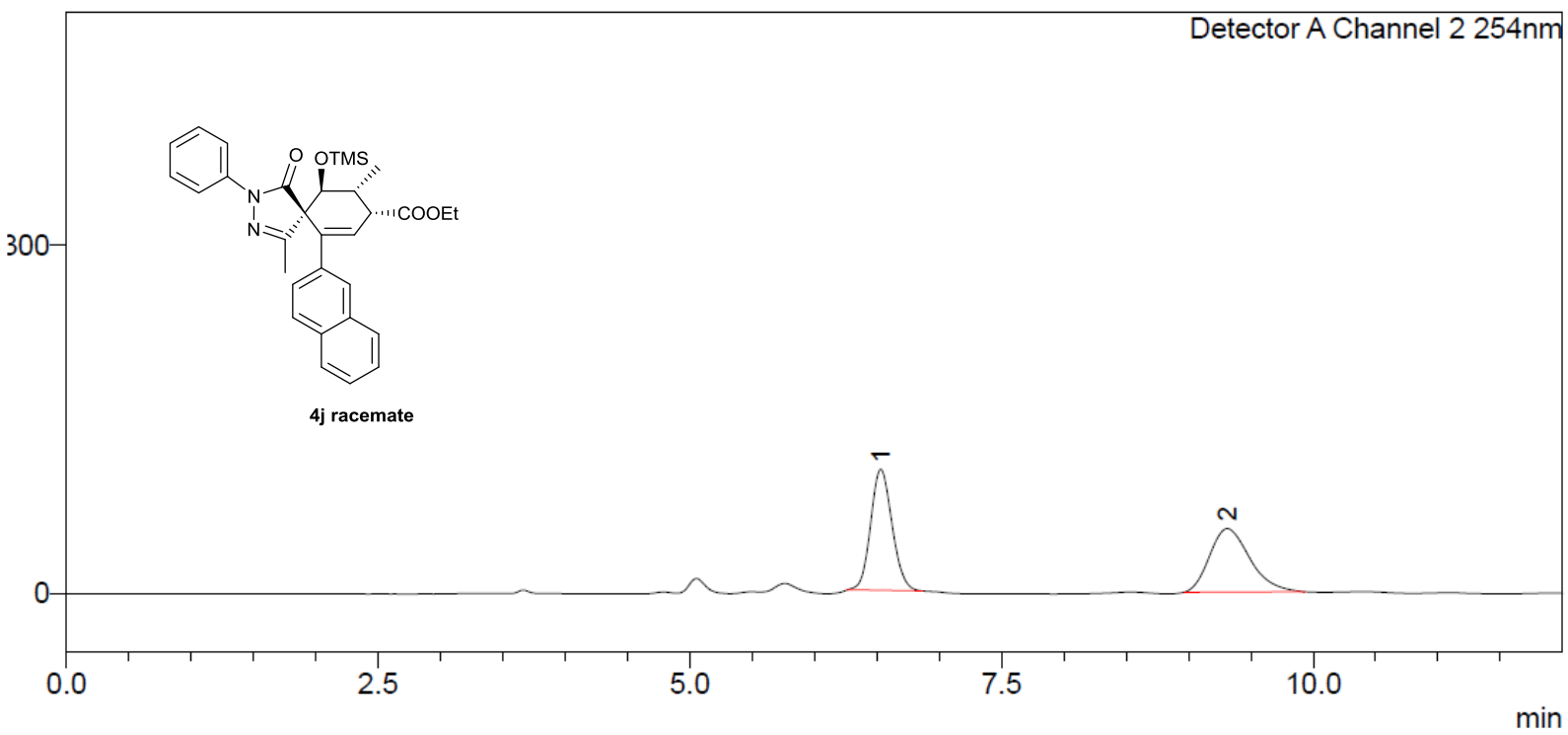

\section{Peak Analysis Report}

Detector A Channel 2 254nm

\begin{tabular}{|c|c|c|c|c|}
\hline No. & Ret. Time & Height $(\mathrm{mAu})$ & Area $\left(\mathrm{mAu}{ }^{*} \mathrm{~min}\right)$ & Rel. Area $(\%)$ \\
\hline 1 & 6.647 & 384 & 4116 & 0.170 \\
\hline 2 & 9.385 & 100440 & 2422975 & 99.830 \\
\hline Total & & 100824 & 2427091 & 100.000 \\
\hline
\end{tabular}

$\mathrm{mV}$

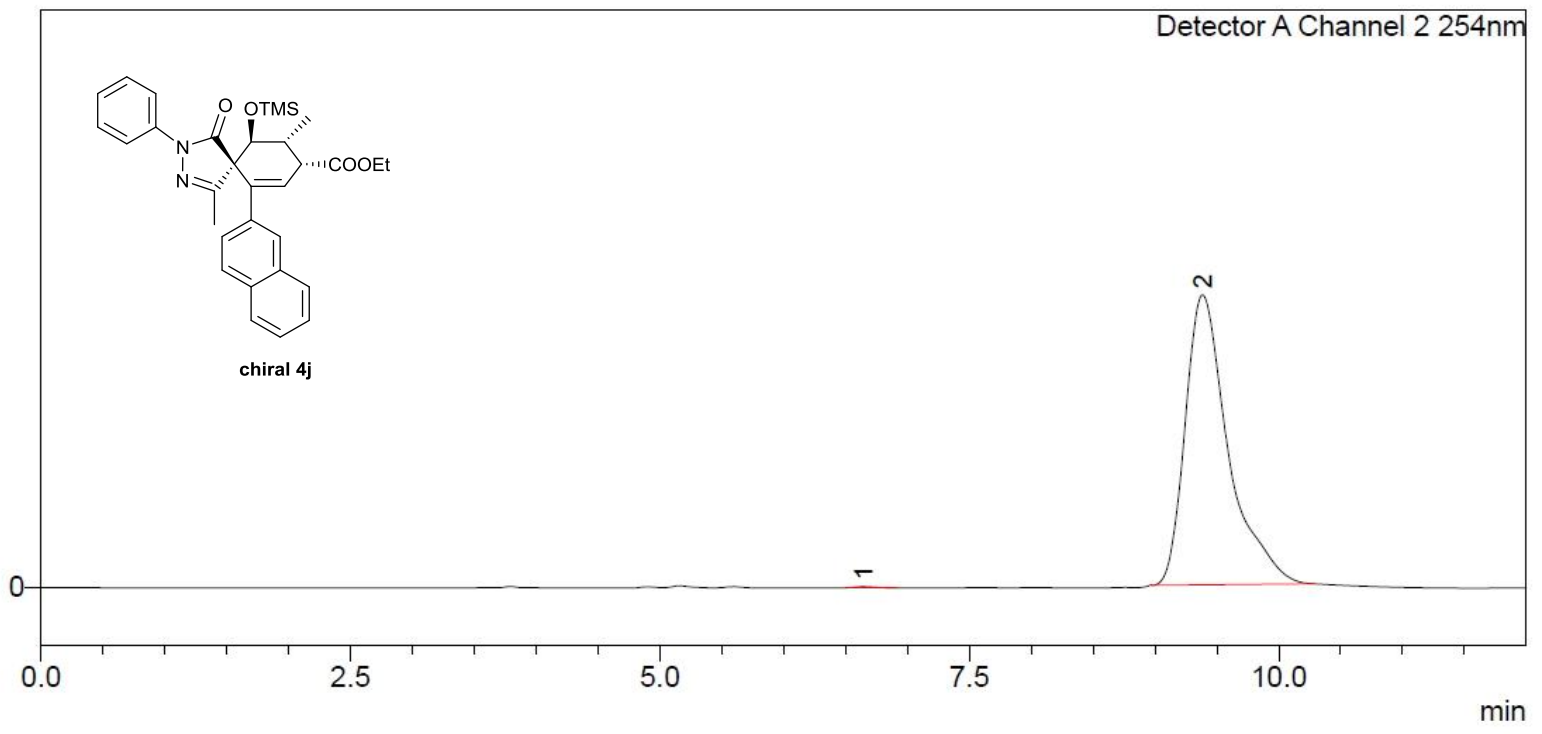




\section{Peak Analysis Report}

Detector A Channel 2 254nm

\begin{tabular}{|c|c|c|c|c|}
\hline No. & Ret. Time & Height $(\mathrm{mAu})$ & Area $\left(\mathrm{mAu}^{*} \mathrm{~min}\right)$ & Rel. Area (\%) \\
\hline 1 & 10.582 & 179470 & 4656010 & 50.480 \\
\hline 2 & 13.178 & 153608 & 4567379 & 49.520 \\
\hline Total & & 333078 & 9223389 & 100.000 \\
\hline
\end{tabular}

$\mathrm{mV}$

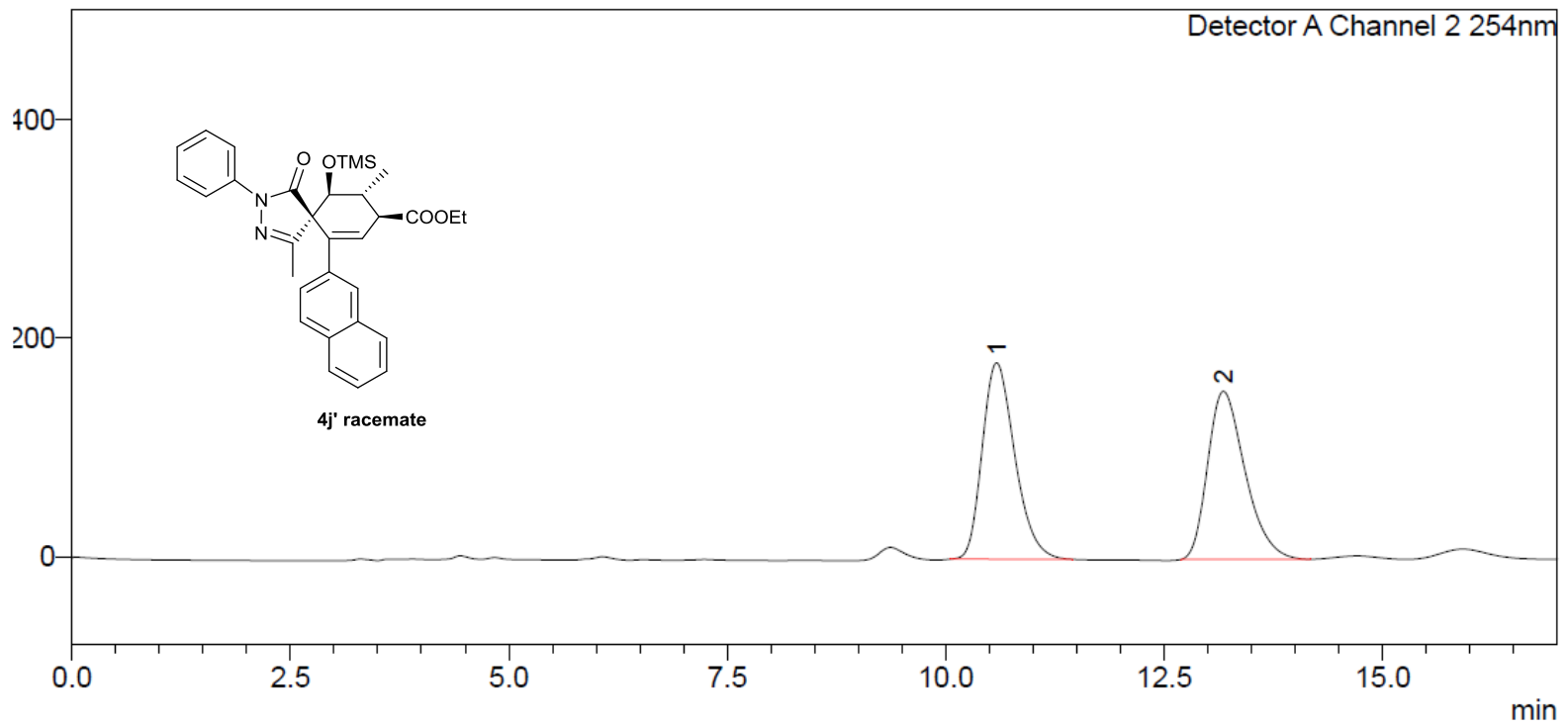

Peak Analysis Report

Detector A Channel $2254 \mathrm{~nm}$

\begin{tabular}{|c|c|c|c|c|}
\hline No. & Ret. Time & Height $(\mathrm{mAu})$ & Area $\left(\mathrm{mAu}{ }^{*} \mathrm{~min}\right)$ & Rel. Area (\%) \\
\hline 1 & 10.611 & 465273 & 11202278 & 46.374 \\
\hline 2 & 13.160 & 430456 & 12954251 & 53.626 \\
\hline Total & & 895728 & 24156529 & 100.000 \\
\hline
\end{tabular}

$\mathrm{mV}$

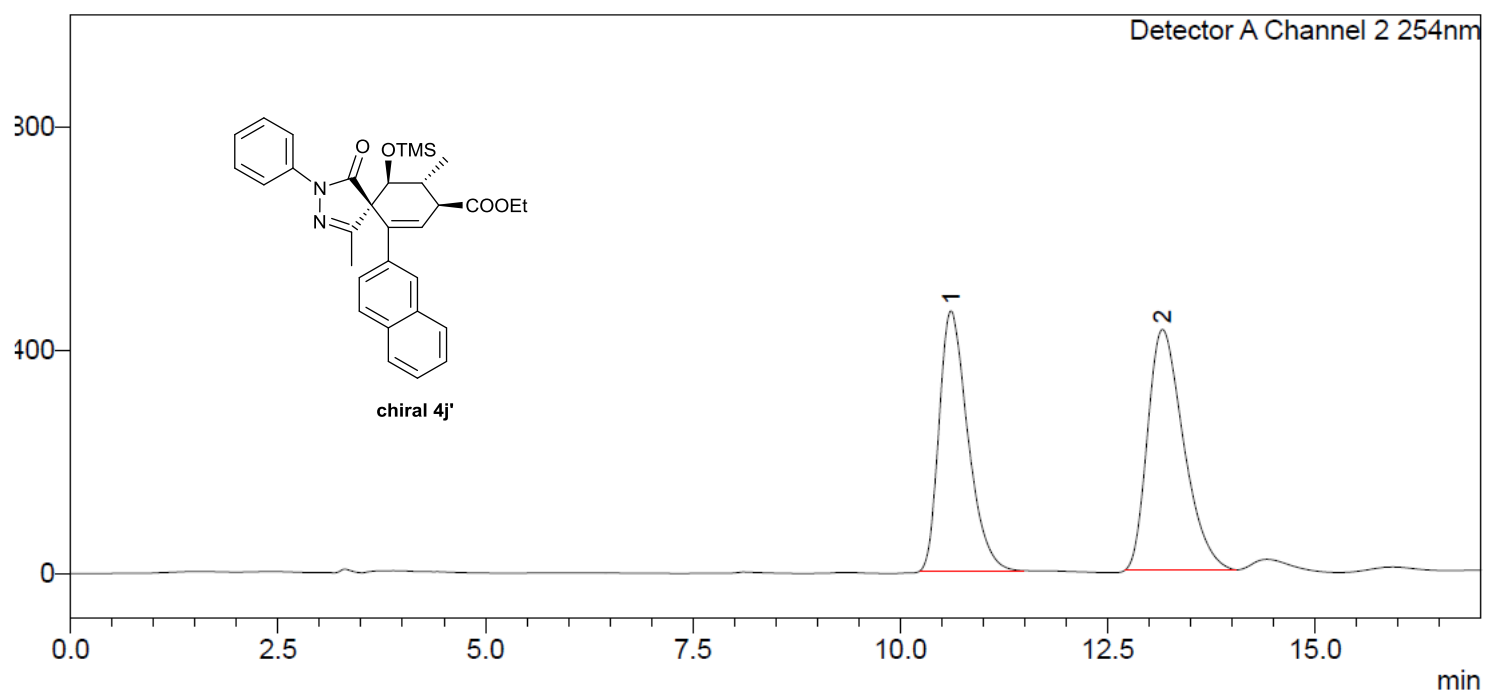



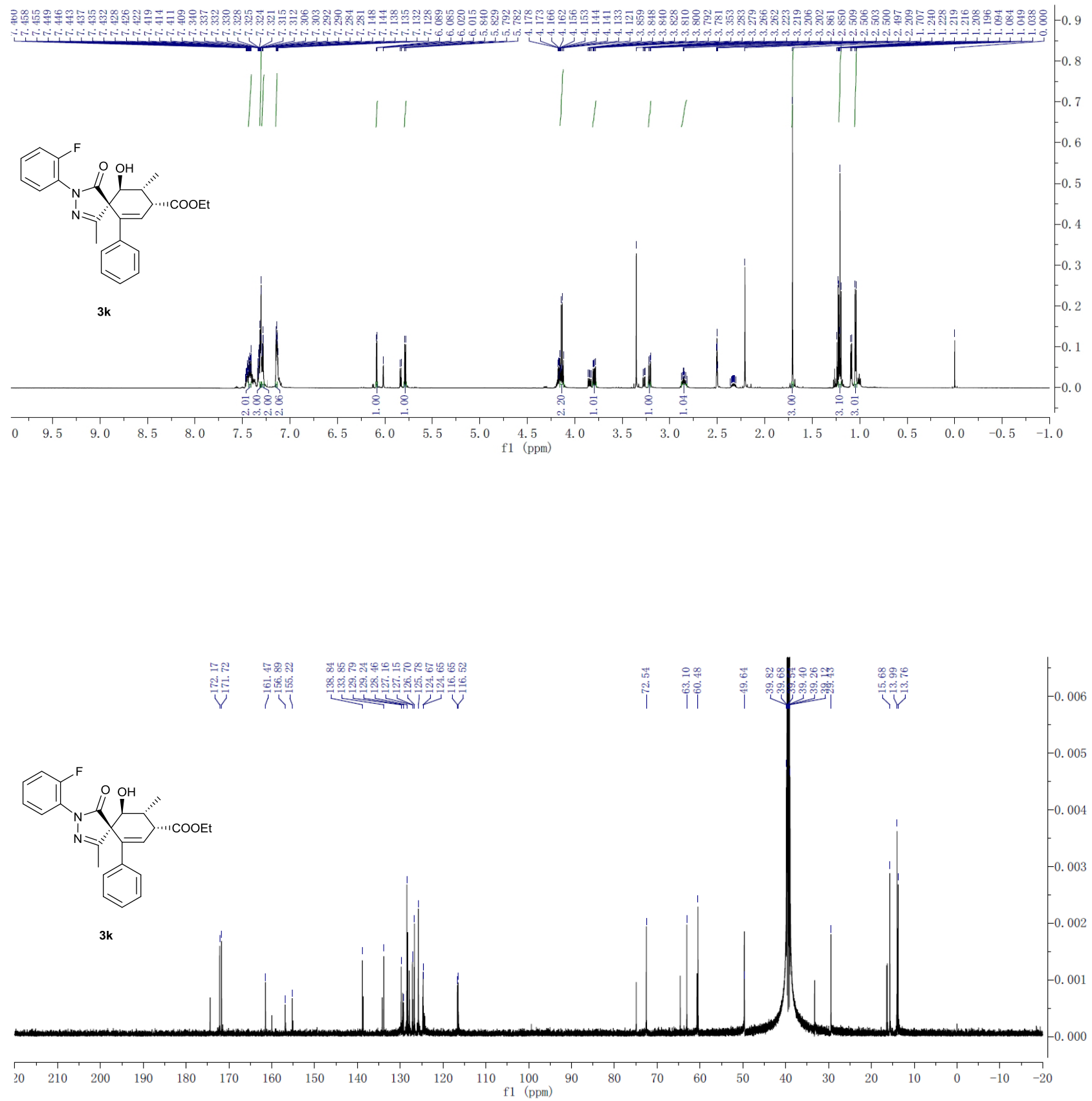


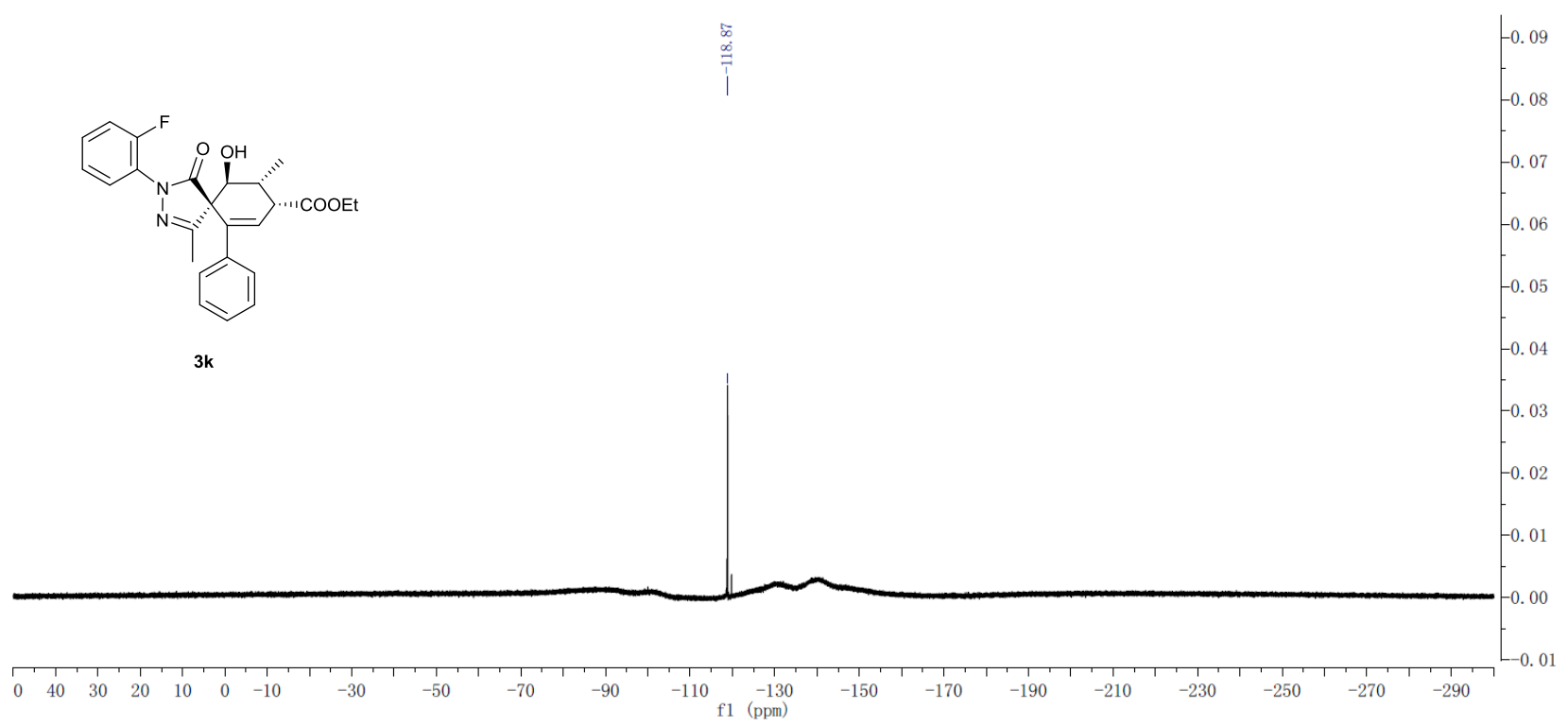



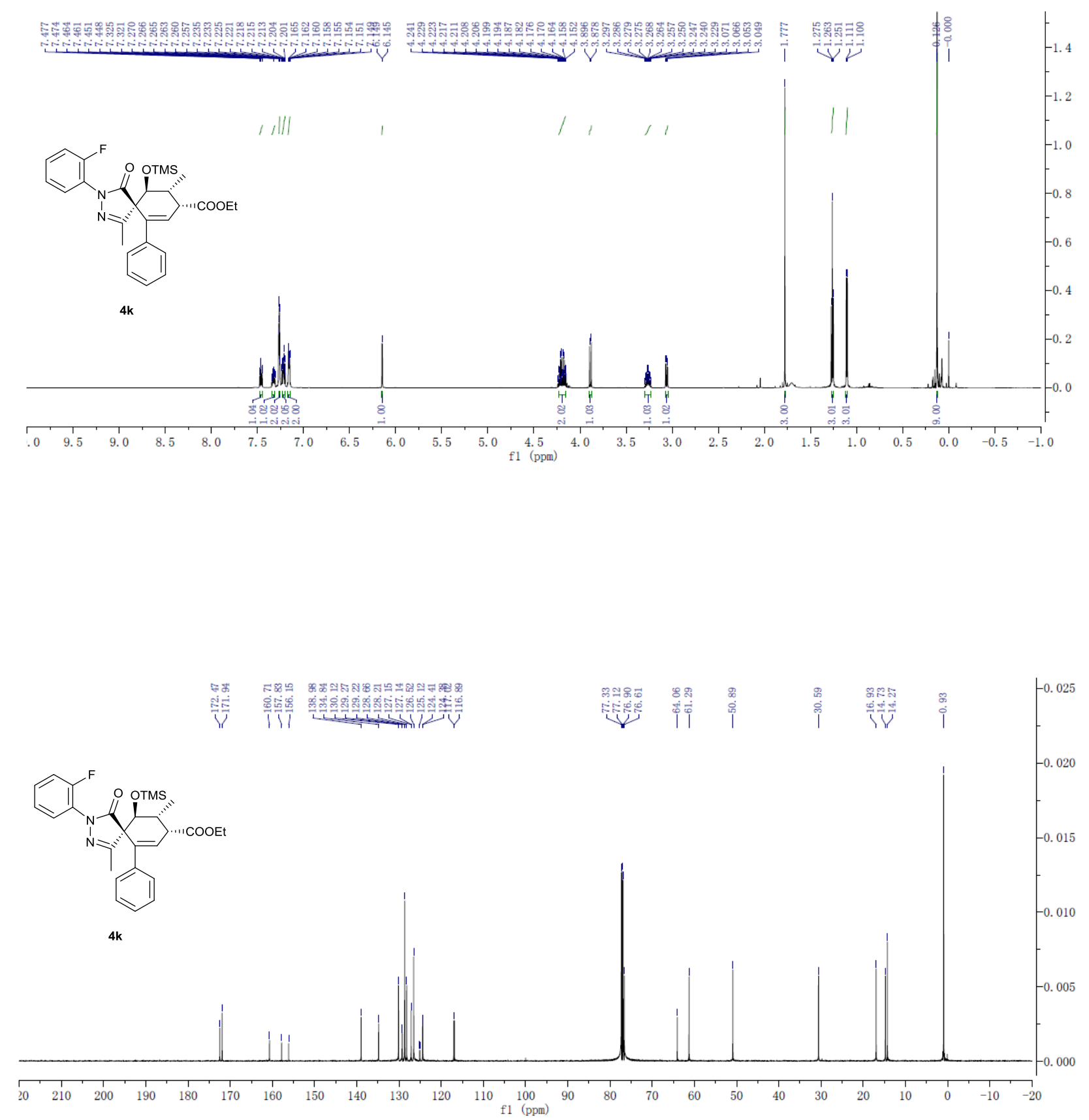


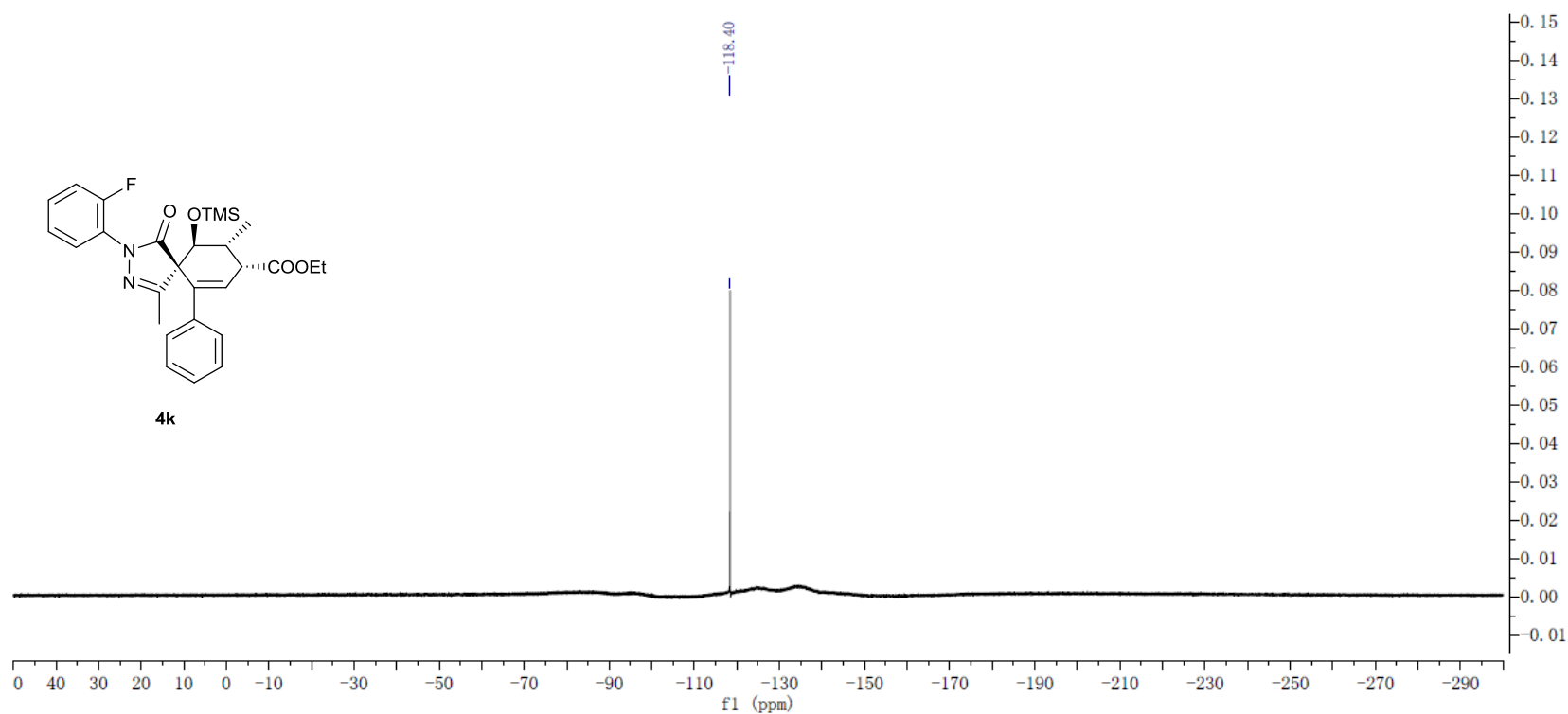



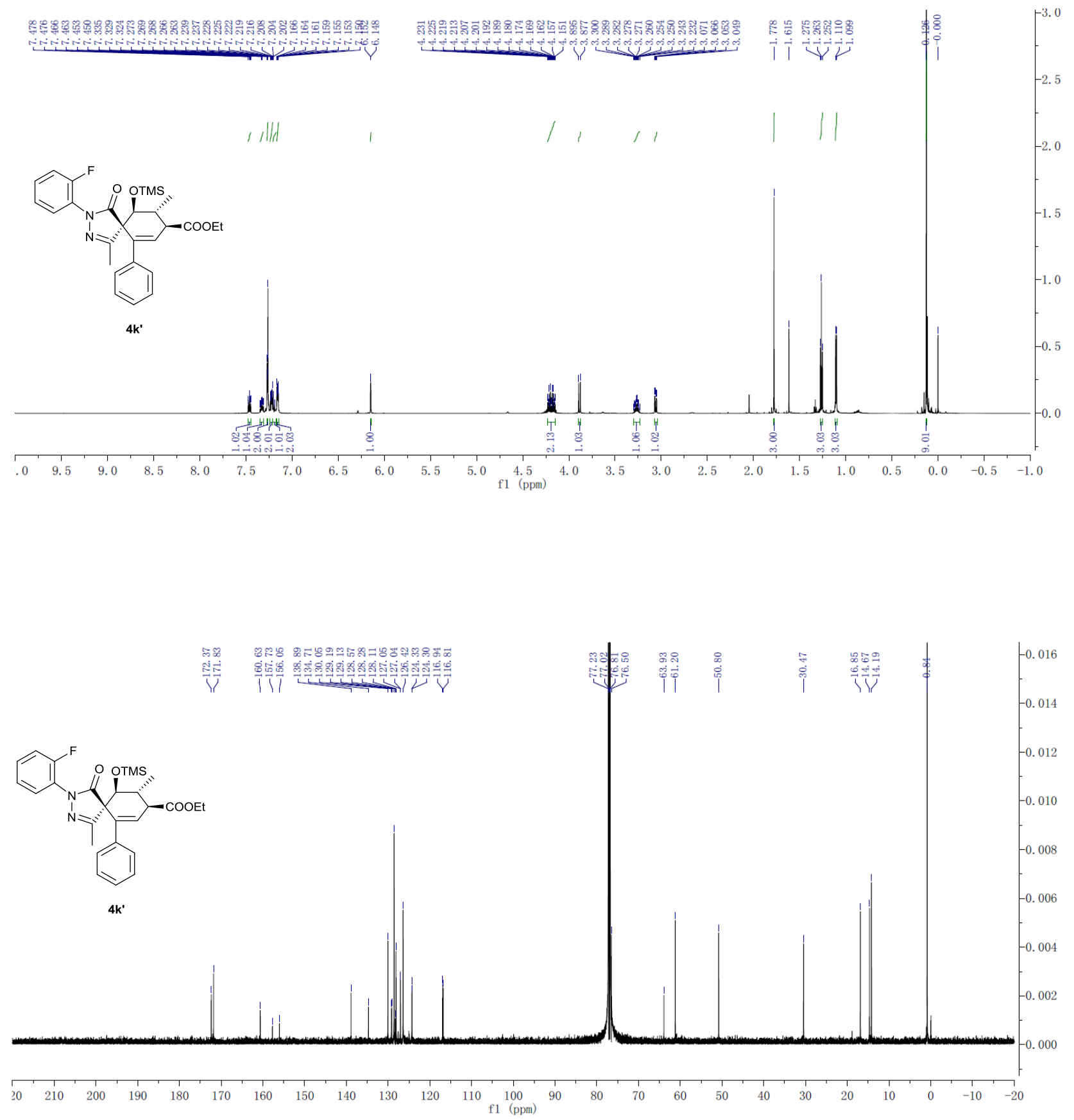


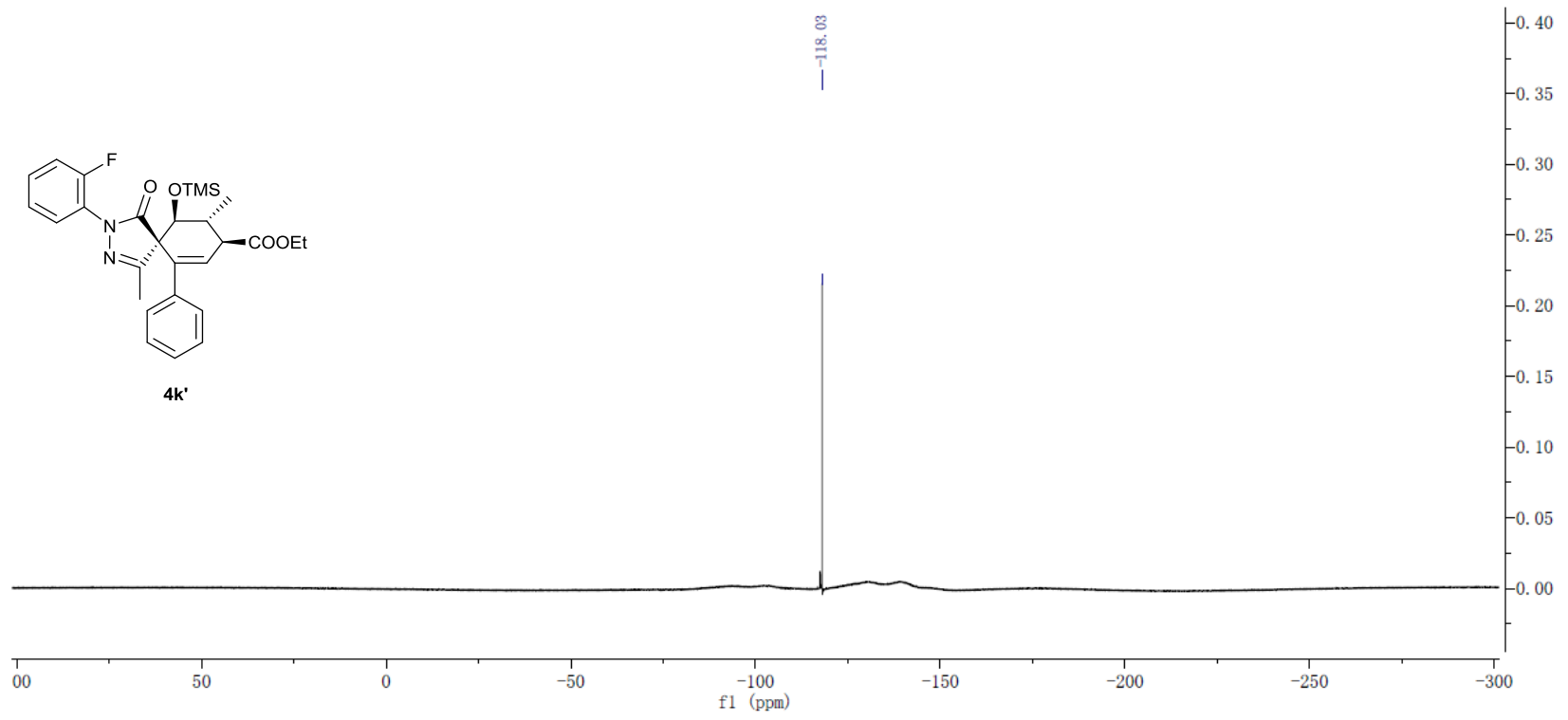


Peak Analysis Report

Detector A Channel $2254 \mathrm{~nm}$

\begin{tabular}{|c|c|c|c|c|}
\hline No. & Ret. Time & Height $(\mathrm{mAu})$ & Area $\left(\mathrm{mAu}^{*} \mathrm{~min}\right)$ & Rel. Area $(\%)$ \\
\hline 1 & 6.844 & 321519 & 5129132 & 49.907 \\
\hline 2 & 8.280 & 256323 & 5148347 & 50.093 \\
\hline Total & & 577842 & 10277479 & 100.000 \\
\hline
\end{tabular}

$\mathrm{mV}$

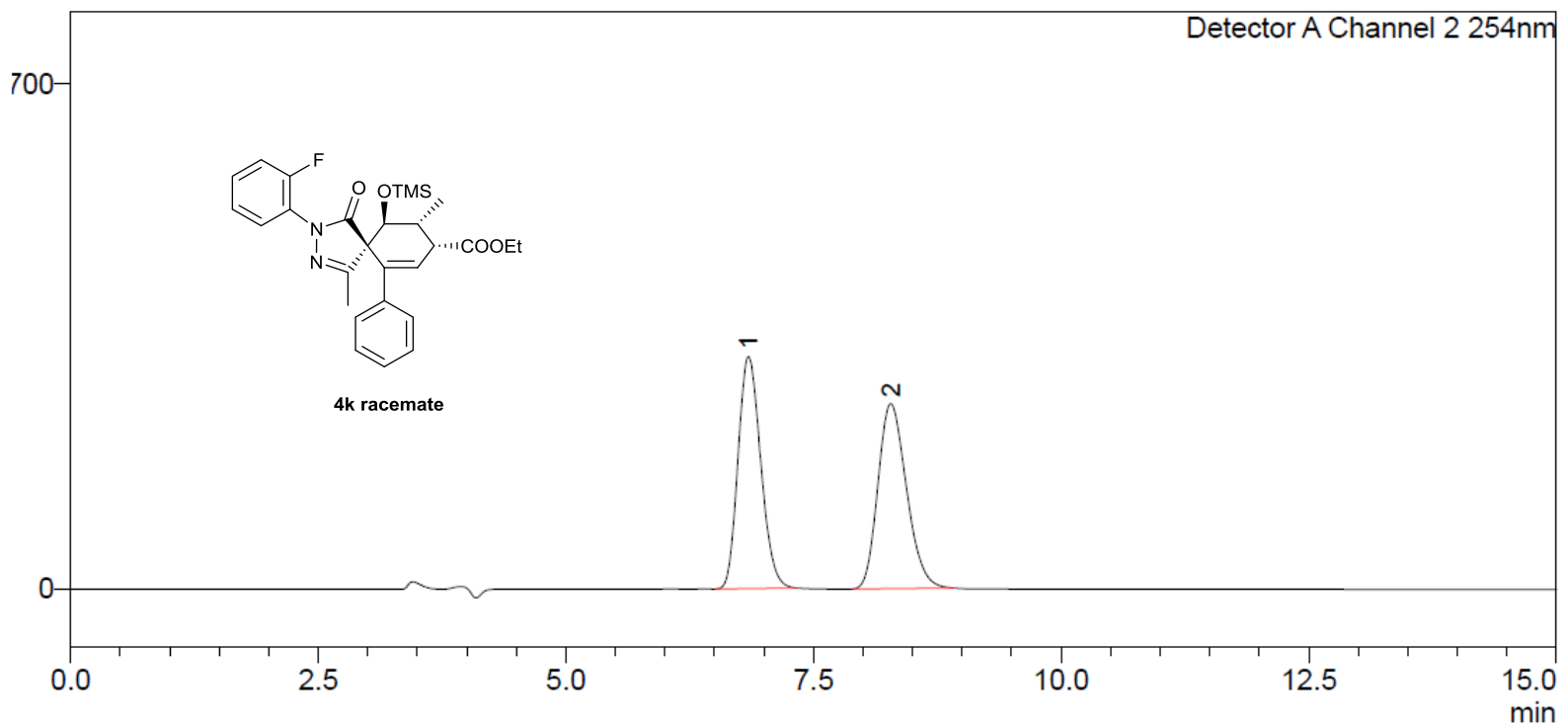

\section{Peak Analysis Report}

Detector A Channel 2 254nm

\begin{tabular}{|c|c|c|c|c|}
\hline No. & Ret. Time & Height $(\mathrm{mAu})$ & Area $\left(\mathrm{mAu}^{*} \mathrm{~min}\right)$ & Rel. Area $(\%)$ \\
\hline 1 & 6.902 & 16738 & 265967 & 2.389 \\
\hline 2 & 8.297 & 493190 & 10864988 & 97.611 \\
\hline Total & & 509927 & 11130954 & 100.000 \\
\hline
\end{tabular}

$\mathrm{mV}$

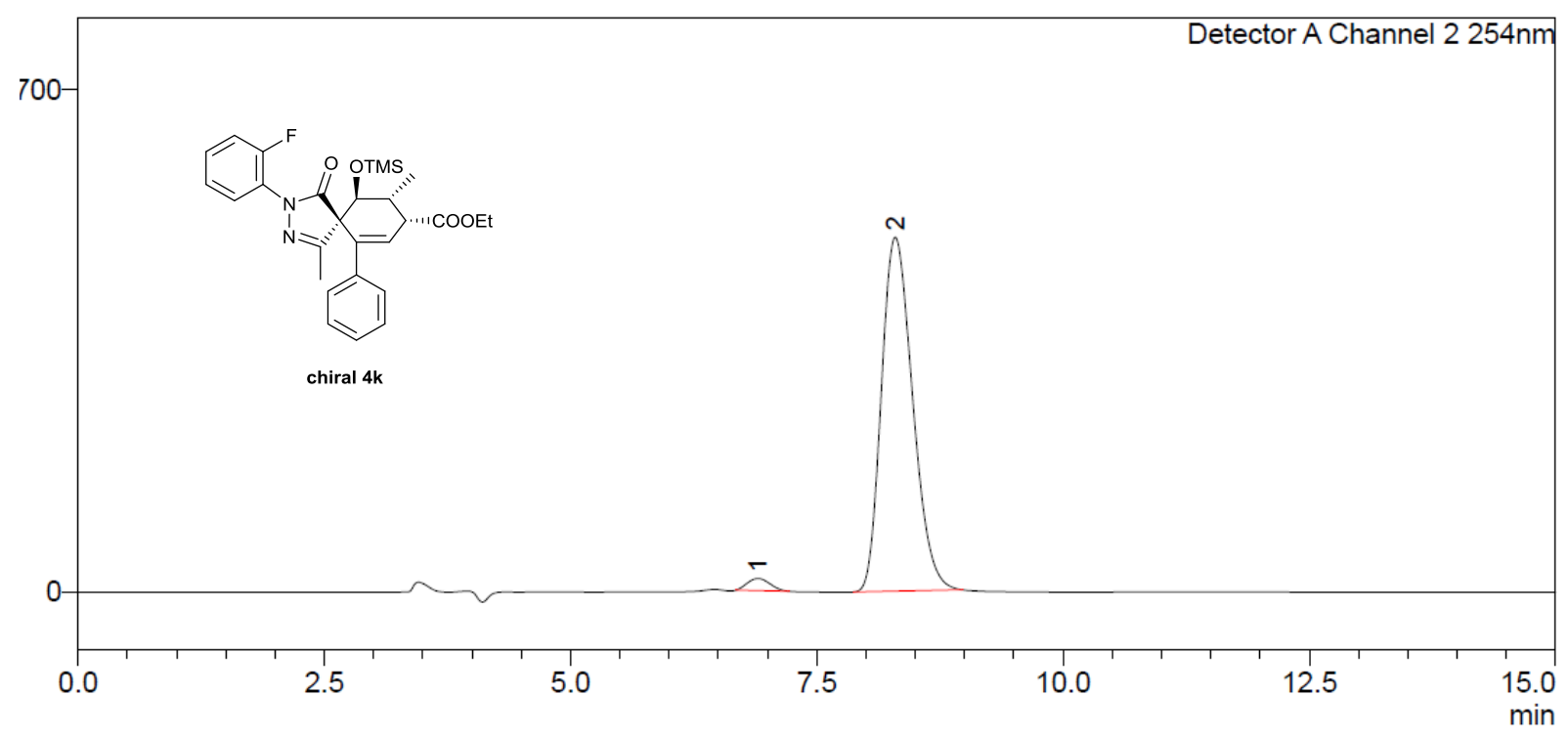




\section{Peak Analysis Report}

Detector A Channel $2254 \mathrm{~nm}$
\begin{tabular}{|c|c|c|c|c|}
\hline No. & Ret. Time & Height (mAu) & Area (mAu*min) & Rel. Area $(\%)$ \\
\hline 1 & 7.889 & 222055 & 3137631 & 50.302 \\
\hline 2 & 16.643 & 95187 & 3099950 & 49.698 \\
\hline Total & & 317242 & 6237581 & 100.000 \\
\hline
\end{tabular}

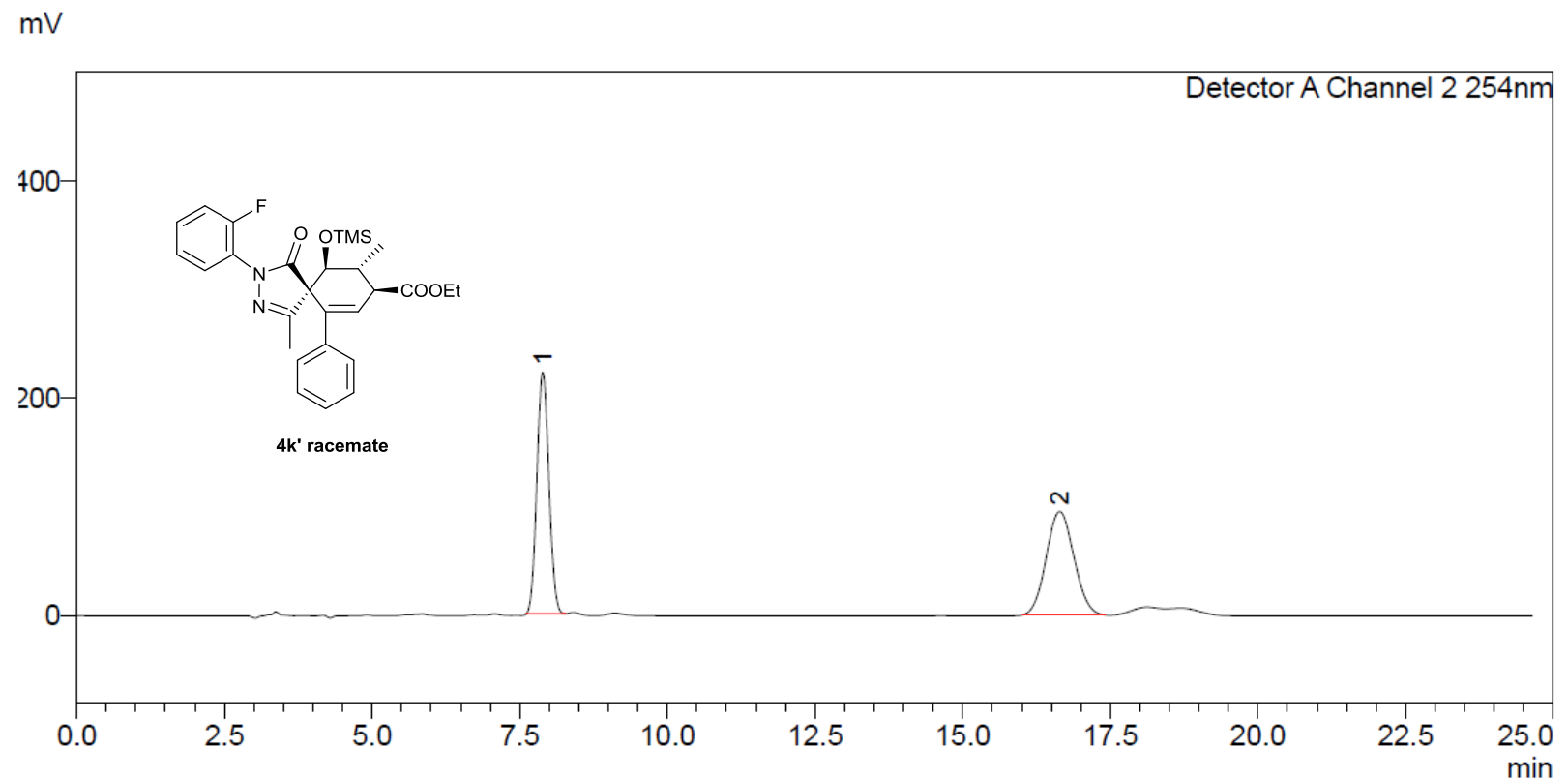

\section{Peak Analysis Report}

Detector A Channel 2 254nm

\begin{tabular}{|c|c|c|c|c|}
\hline No. & Ret. Time & Height $(\mathrm{mAu})$ & Area $\left(\mathrm{mAu}^{*} \mathrm{~min}\right)$ & Rel. Area (\%) \\
\hline 1 & 7.900 & 182282 & 2618672 & 91.075 \\
\hline 2 & 16.667 & 9138 & 256605 & 8.925 \\
\hline Total & & 191420 & 2875277 & 100.000 \\
\hline
\end{tabular}

$\mathrm{mV}$

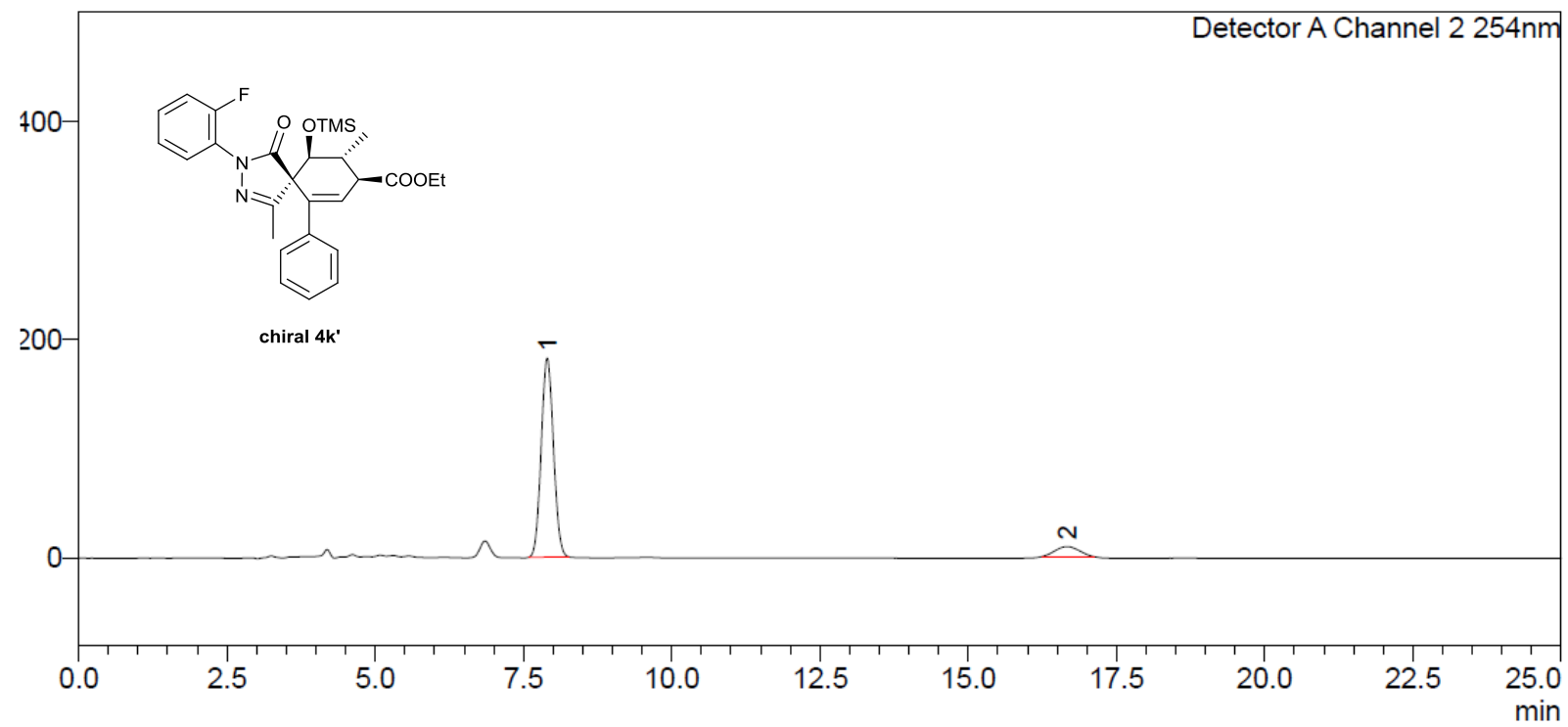



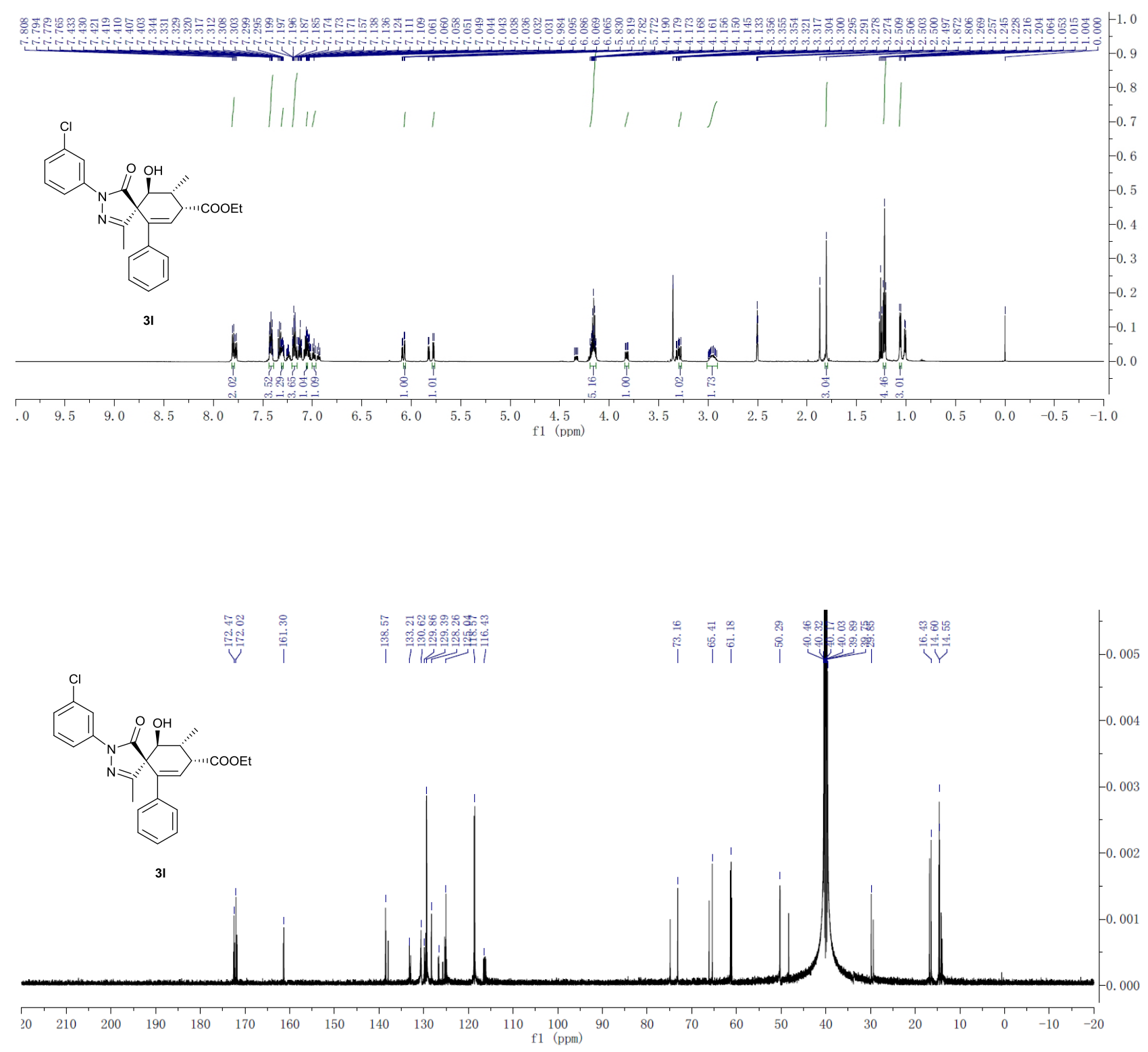

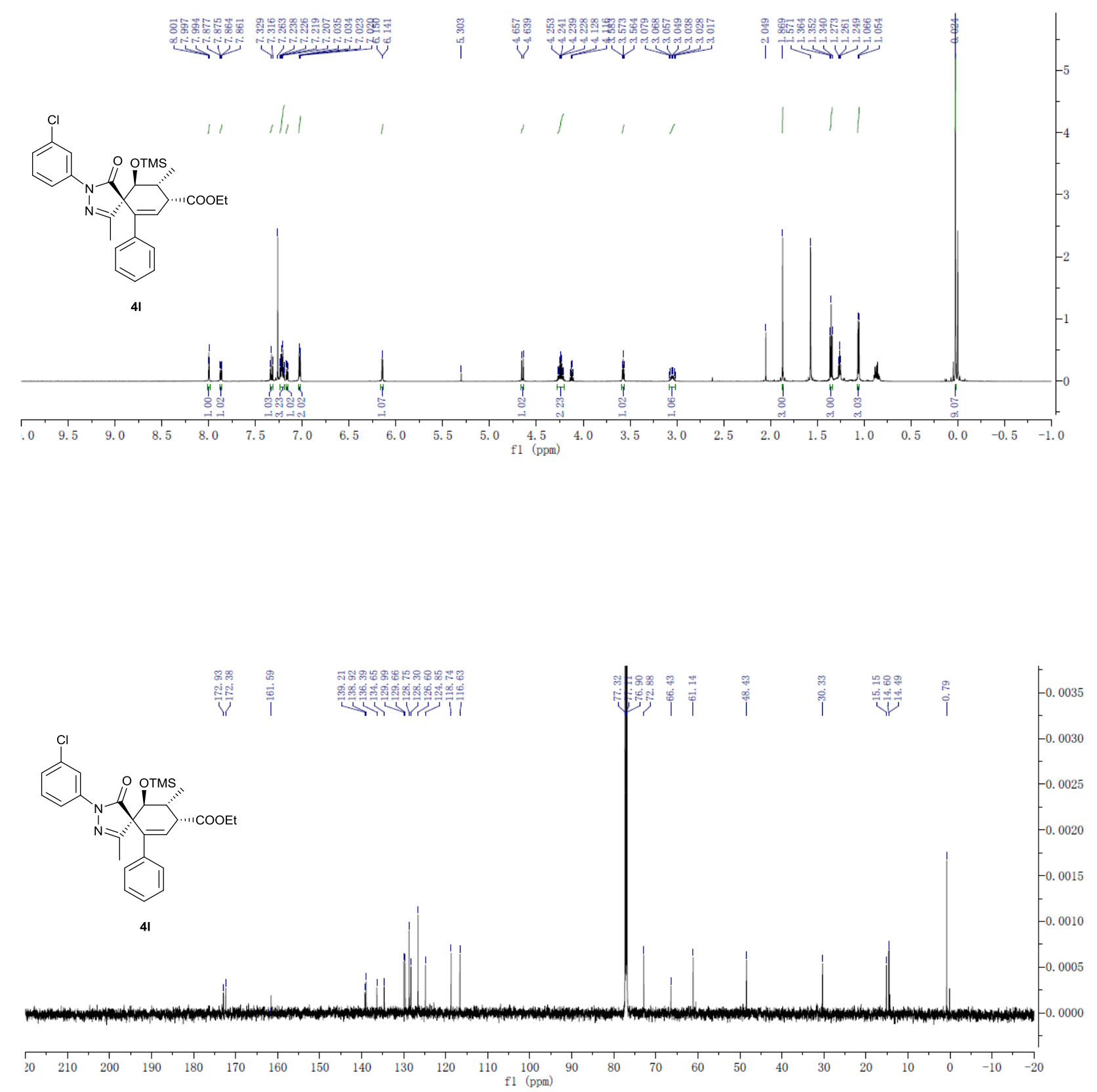

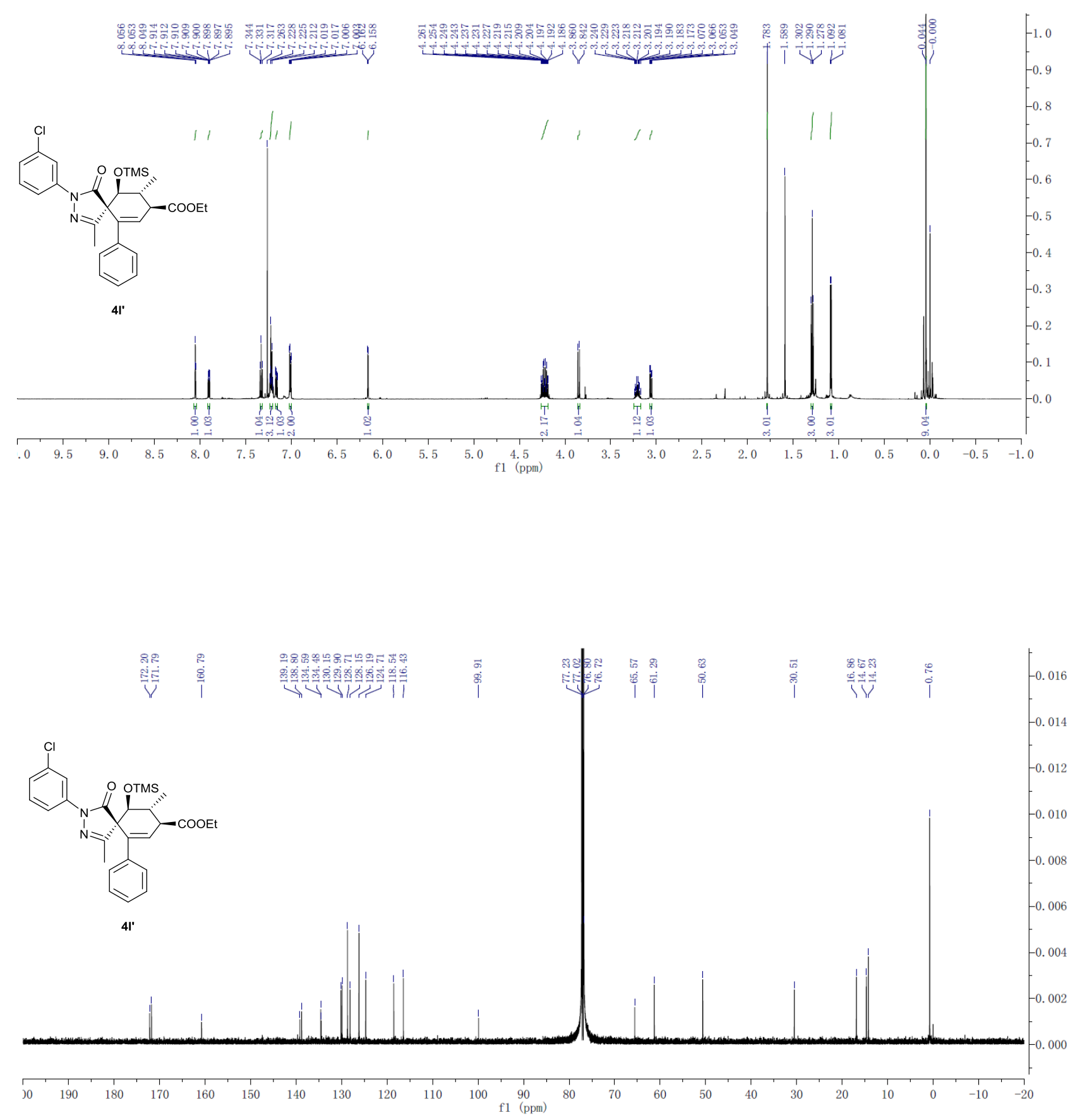


\section{Peak Analysis Report}

Detector A Channel 2 254nm

\begin{tabular}{|c|c|c|c|c|}
\hline No. & Ret. Time & Height $(\mathrm{mAu})$ & Area $\left(\mathrm{mAu}^{*} \min \right)$ & Rel. Area (\%) \\
\hline 1 & 4.417 & 1826016 & 15596972 & 55.234 \\
\hline 2 & 5.173 & 1404990 & 12640997 & 44.766 \\
\hline Total & & 3231006 & 28237969 & 100.000 \\
\hline
\end{tabular}

$\mathrm{mV}$

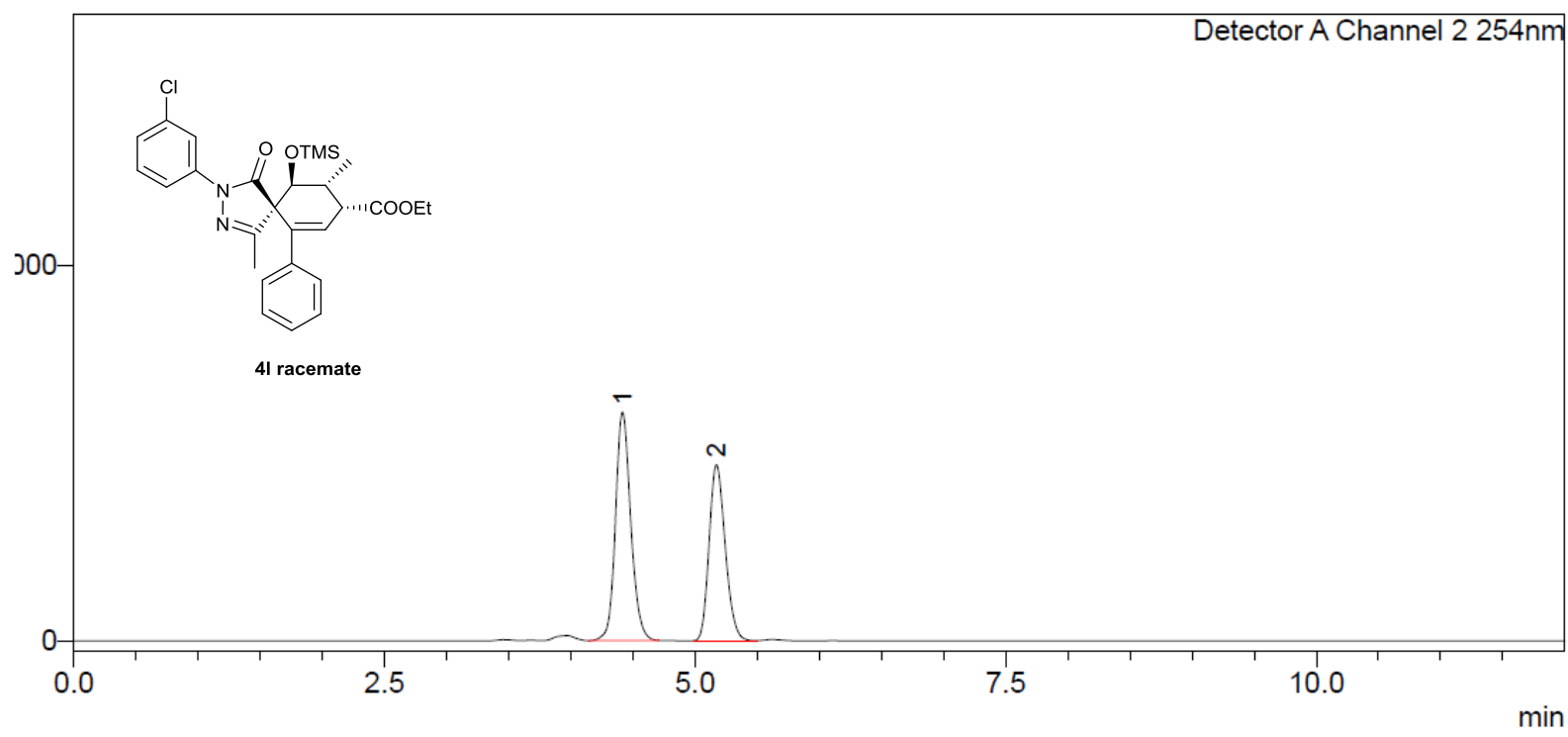

Peak Analysis Report

Detector A Channel 2 254nm

Detector A Channel 2 254nm
\begin{tabular}{|c|c|c|c|c|}
\hline No. & Ret. Time & Height (mAu) & Area $\left(\mathrm{mAu}^{*} \mathrm{~min}\right)$ & Rel. Area $(\%)$ \\
\hline 1 & 4.739 & 93550 & 702610 & 2.854 \\
\hline 2 & 5.462 & 2676671 & 23918558 & 97.146 \\
\hline Total & & 2770221 & 24621168 & 100.000 \\
\hline
\end{tabular}

$\mathrm{mV}$

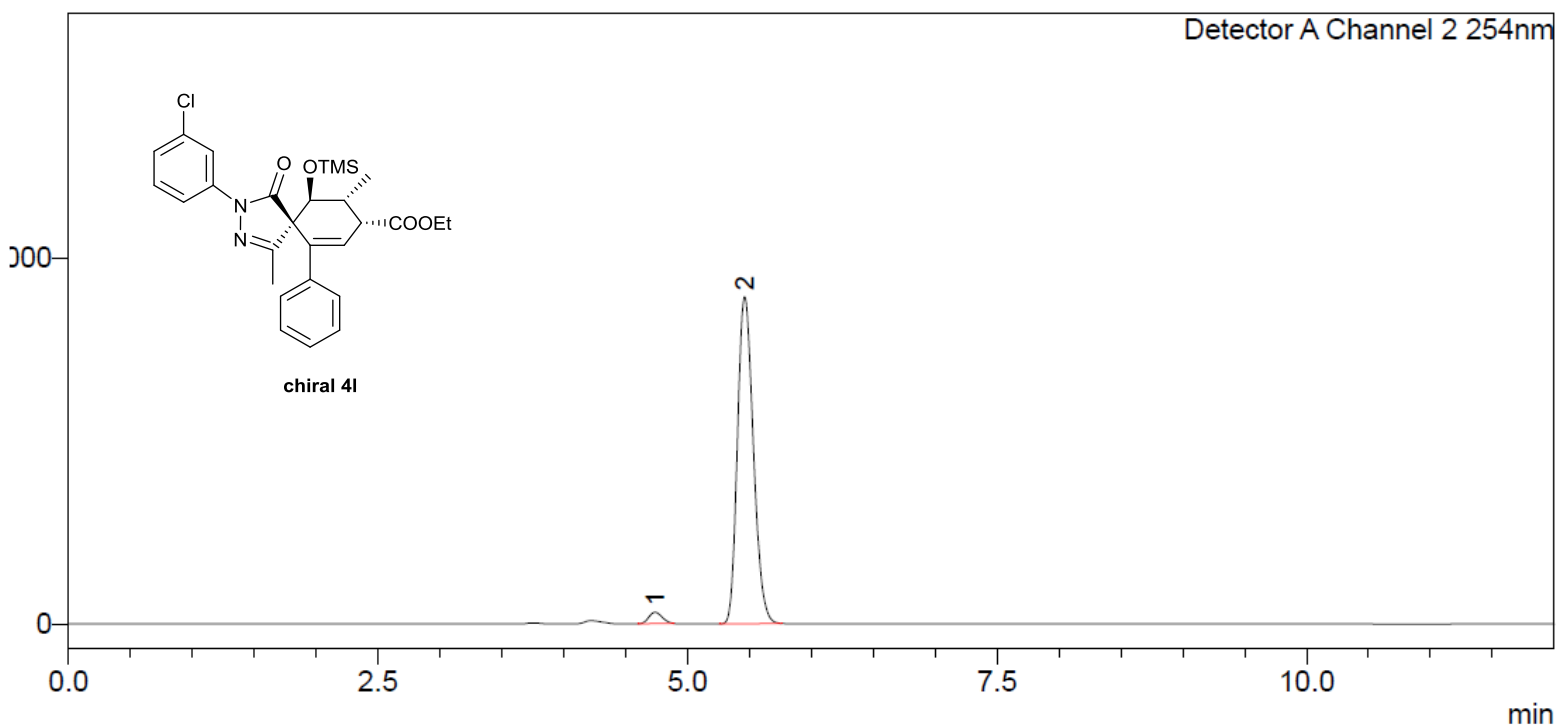


Peak Analysis Report

Detector A Channel 2 254nm

\begin{tabular}{|c|c|c|c|c|}
\hline No. & Ret. Time & Height $(\mathrm{mAu})$ & Area $\left(\mathrm{mAu}^{*} \mathrm{~min}\right)$ & Rel. Area (\%) \\
\hline 1 & 15.920 & 44994 & 1821774 & 50.818 \\
\hline 2 & 17.830 & 39744 & 1763146 & 49.182 \\
\hline Total & & 84738 & 3584920 & 100.000 \\
\hline
\end{tabular}

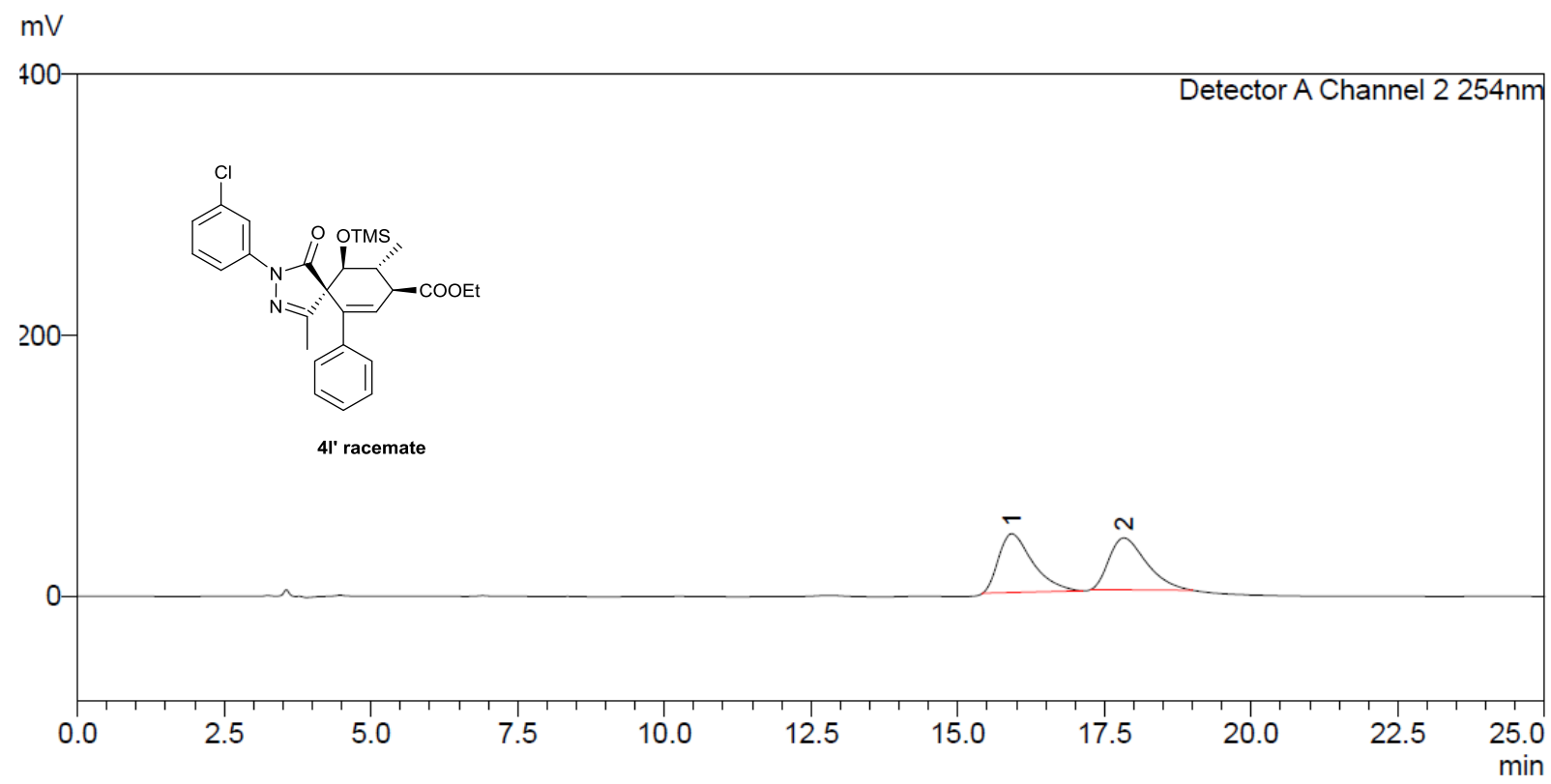

Peak Analysis Report

Detector A Channel $2254 \mathrm{~nm}$

\begin{tabular}{|c|c|c|c|c|}
\hline No. & Ret. Time & Height $(\mathrm{mAu})$ & Area $\left(\mathrm{mAu}^{*} \mathrm{~min}\right)$ & Rel. Area (\%) \\
\hline 1 & 15.782 & 94466 & 3614296 & 47.936 \\
\hline 2 & 17.444 & 89865 & 3925536 & 52.064 \\
\hline Total & & 184331 & 7539832 & 100.000 \\
\hline
\end{tabular}

$\mathrm{mV}$

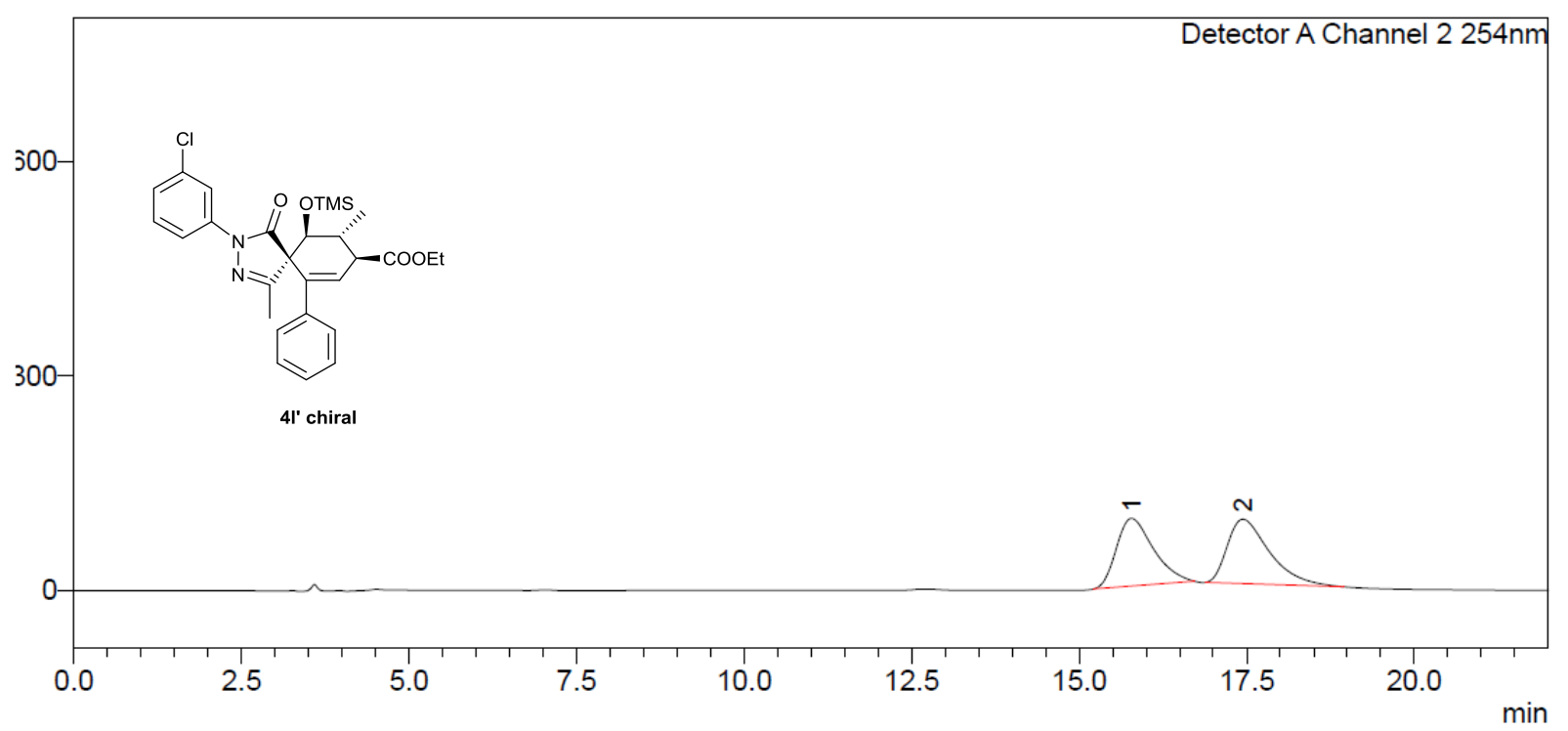



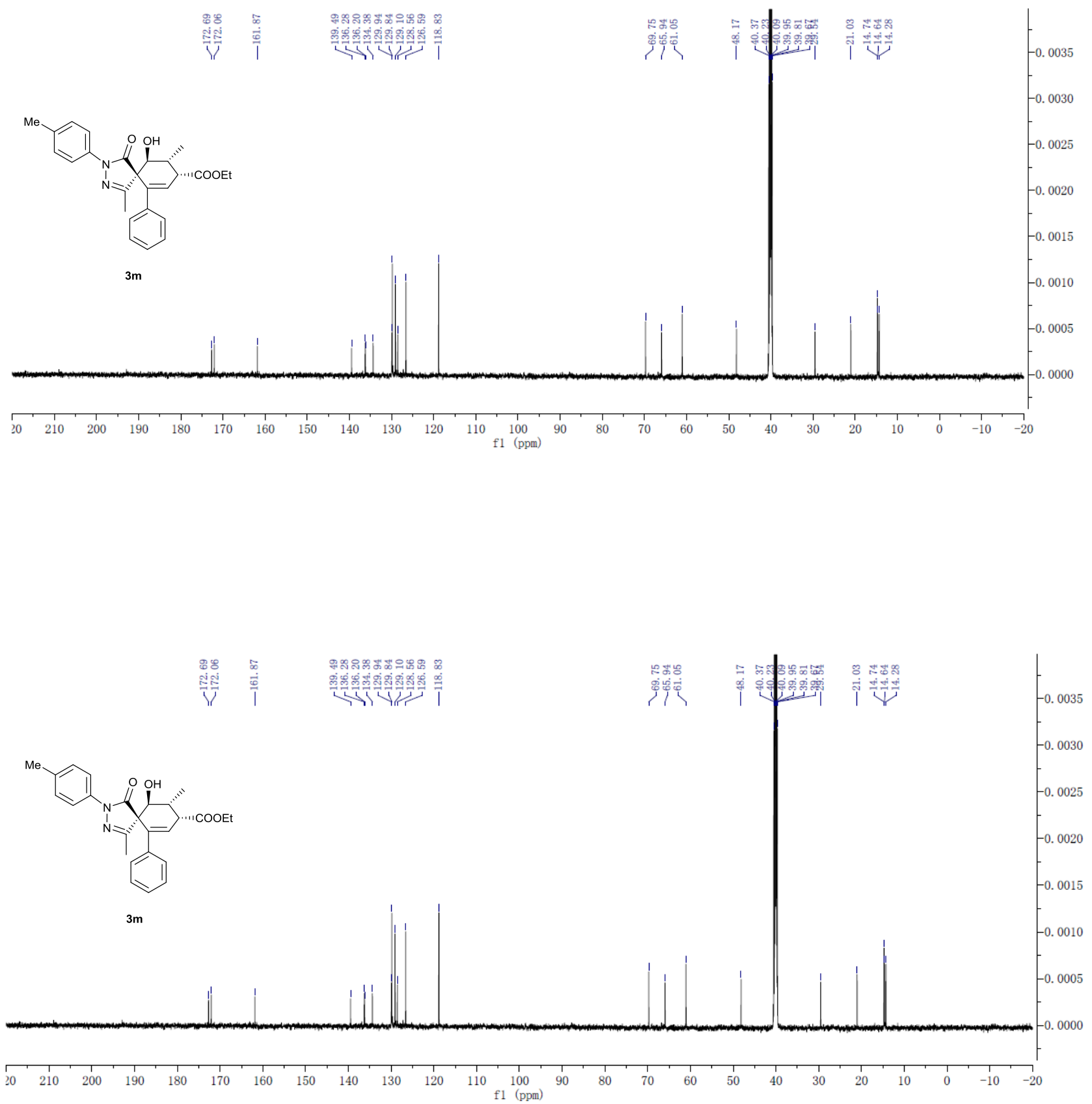

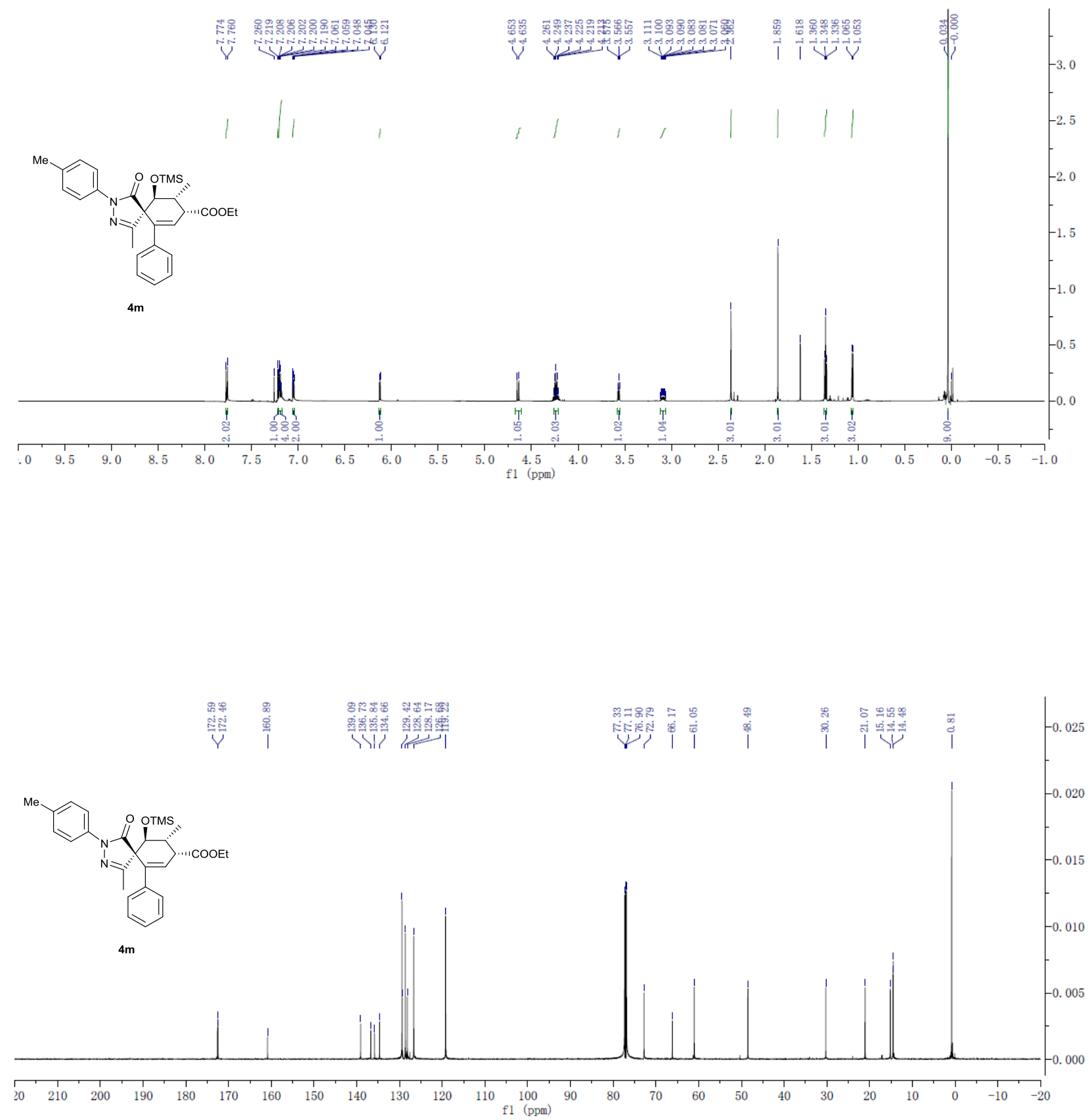

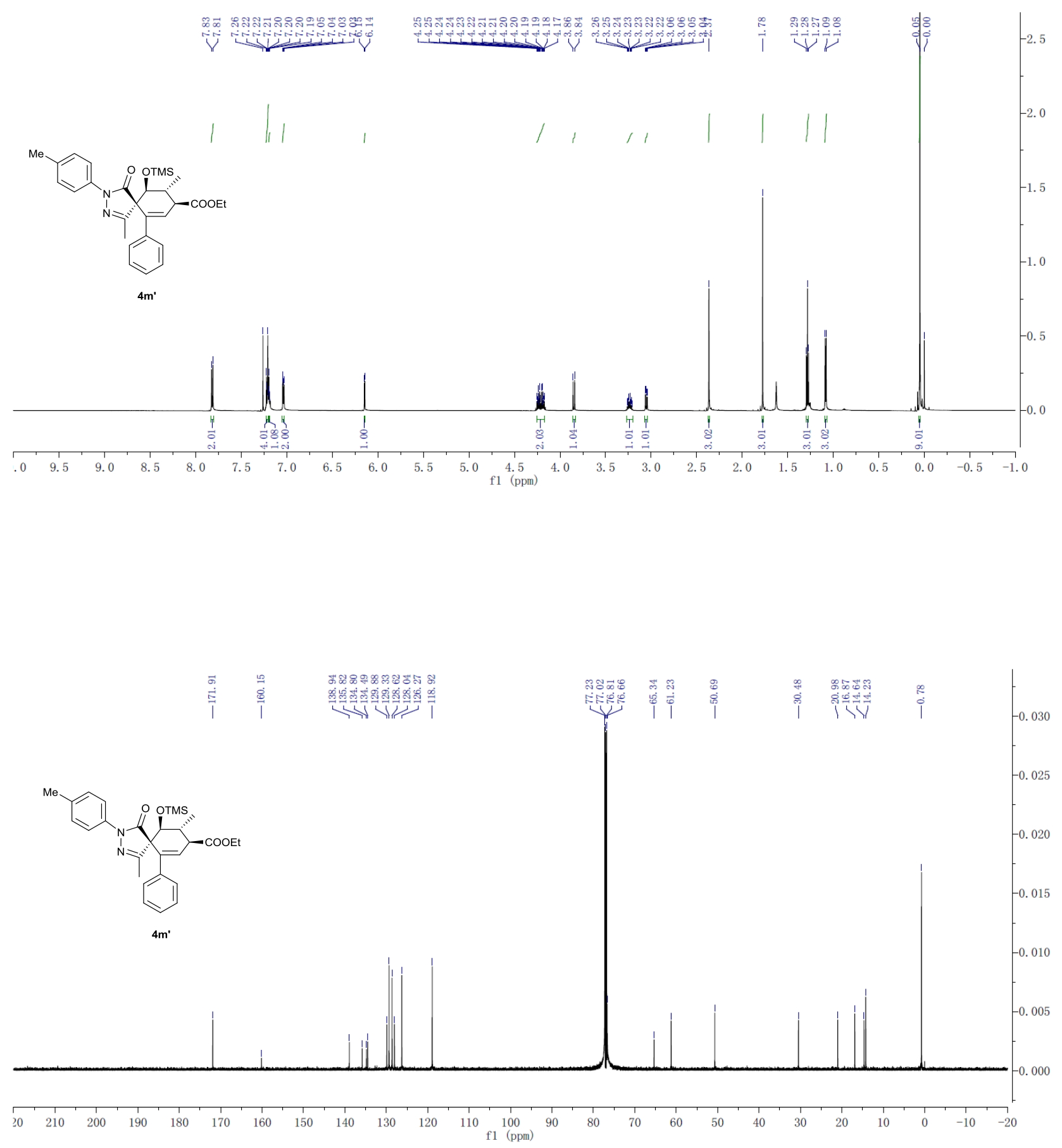


\section{Peak Analysis Report}

Detector A Channel $2254 \mathrm{~nm}$

\begin{tabular}{|c|c|c|c|c|}
\hline No. & Ret. Time & Height $(\mathrm{mAu})$ & Area $\left(\mathrm{mAu}^{*} \mathrm{~min}\right)$ & Rel. Area $(\%)$ \\
\hline 1 & 6.142 & 203440 & 2226360 & 49.338 \\
\hline 2 & 20.709 & 58122 & 2286117 & 50.662 \\
\hline Total & & 261562 & 4512477 & 100.000 \\
\hline
\end{tabular}

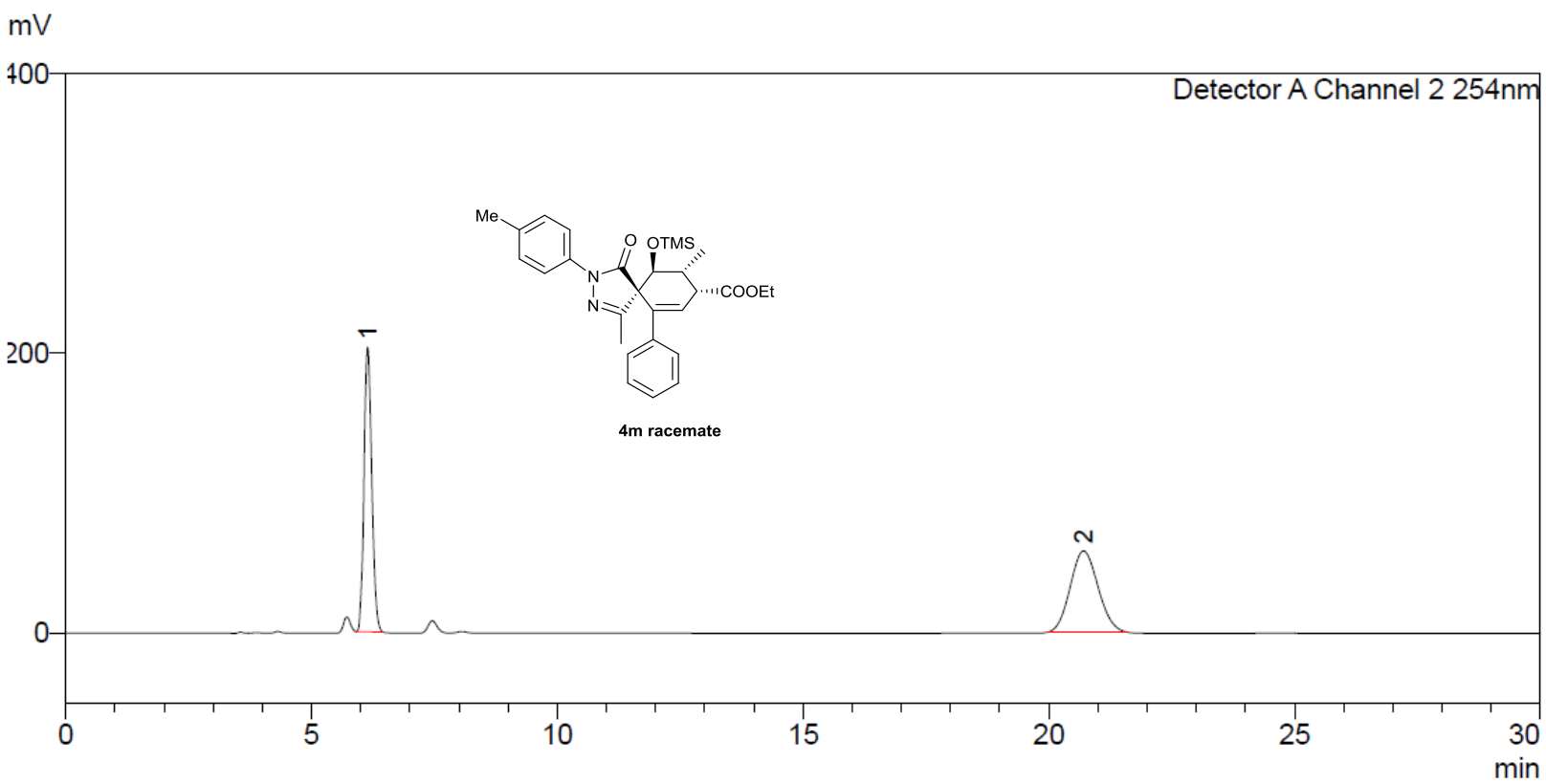

Peak Analysis Report

Detector A Channel 2 254nm

\begin{tabular}{|c|c|c|c|c|}
\hline No. & Ret. Time & Height $(\mathrm{mAu})$ & Area $\left(\mathrm{mAu}^{*} \mathrm{~min}\right)$ & Rel. Area $(\%)$ \\
\hline 1 & 5.279 & 140 & 1295 & 0.022 \\
\hline 2 & 19.847 & 143170 & 5758541 & 99.978 \\
\hline Total & & 143310 & 5759835 & 100.000 \\
\hline
\end{tabular}

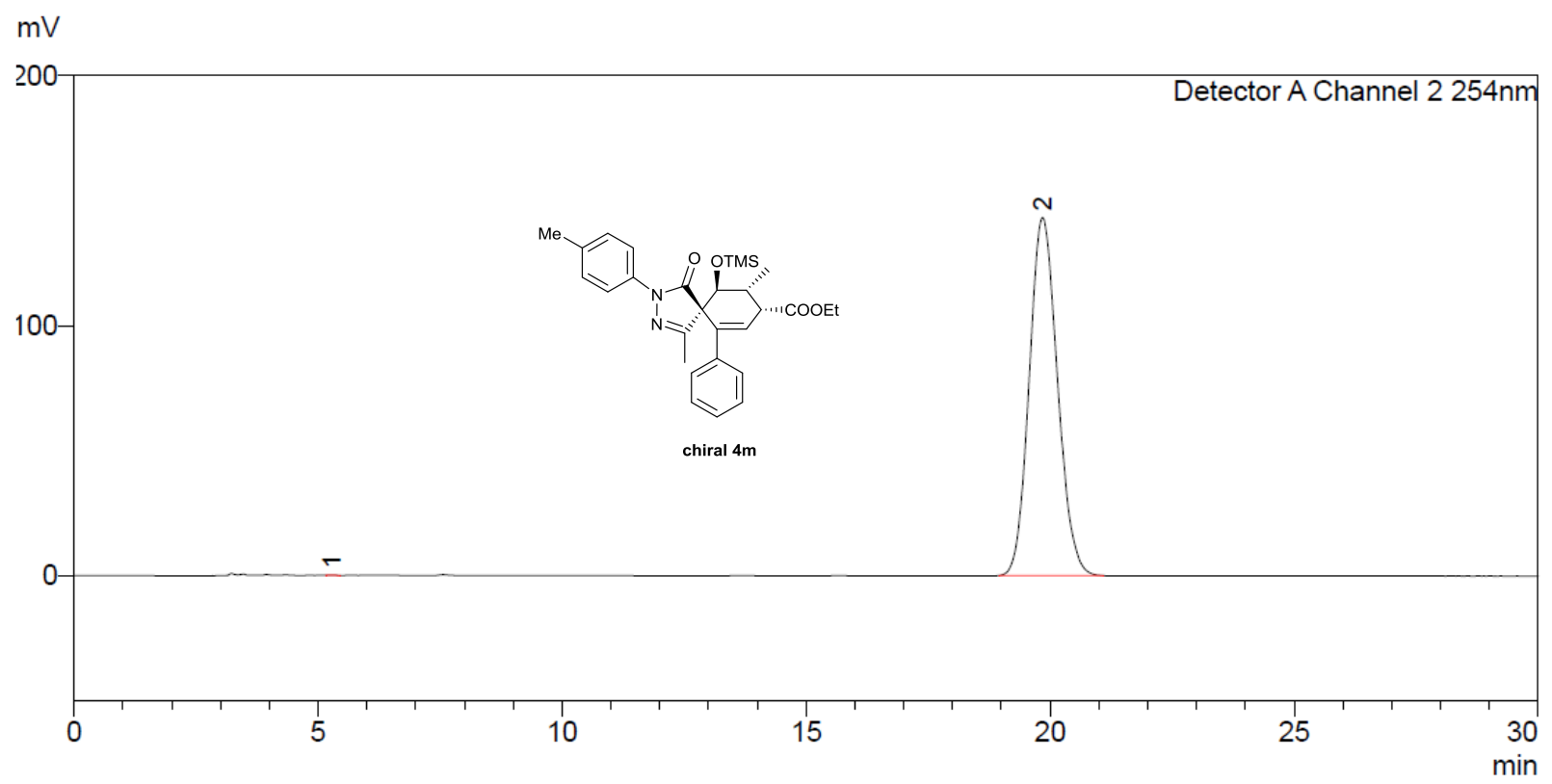




\section{Peak Analysis Report}

Detector A Channel $2254 \mathrm{~nm}$

\begin{tabular}{|c|c|c|c|c|}
\hline No. & Ret. Time & Height $(\mathrm{mAu})$ & Area $\left(\mathrm{mAu}^{*} \mathrm{~min}\right)$ & Rel. Area (\%) \\
\hline 1 & 11.547 & 122424 & 3082190 & 50.583 \\
\hline 2 & 12.667 & 109713 & 3011187 & 49.417 \\
\hline Total & & 232137 & 6093378 & 100.000 \\
\hline
\end{tabular}

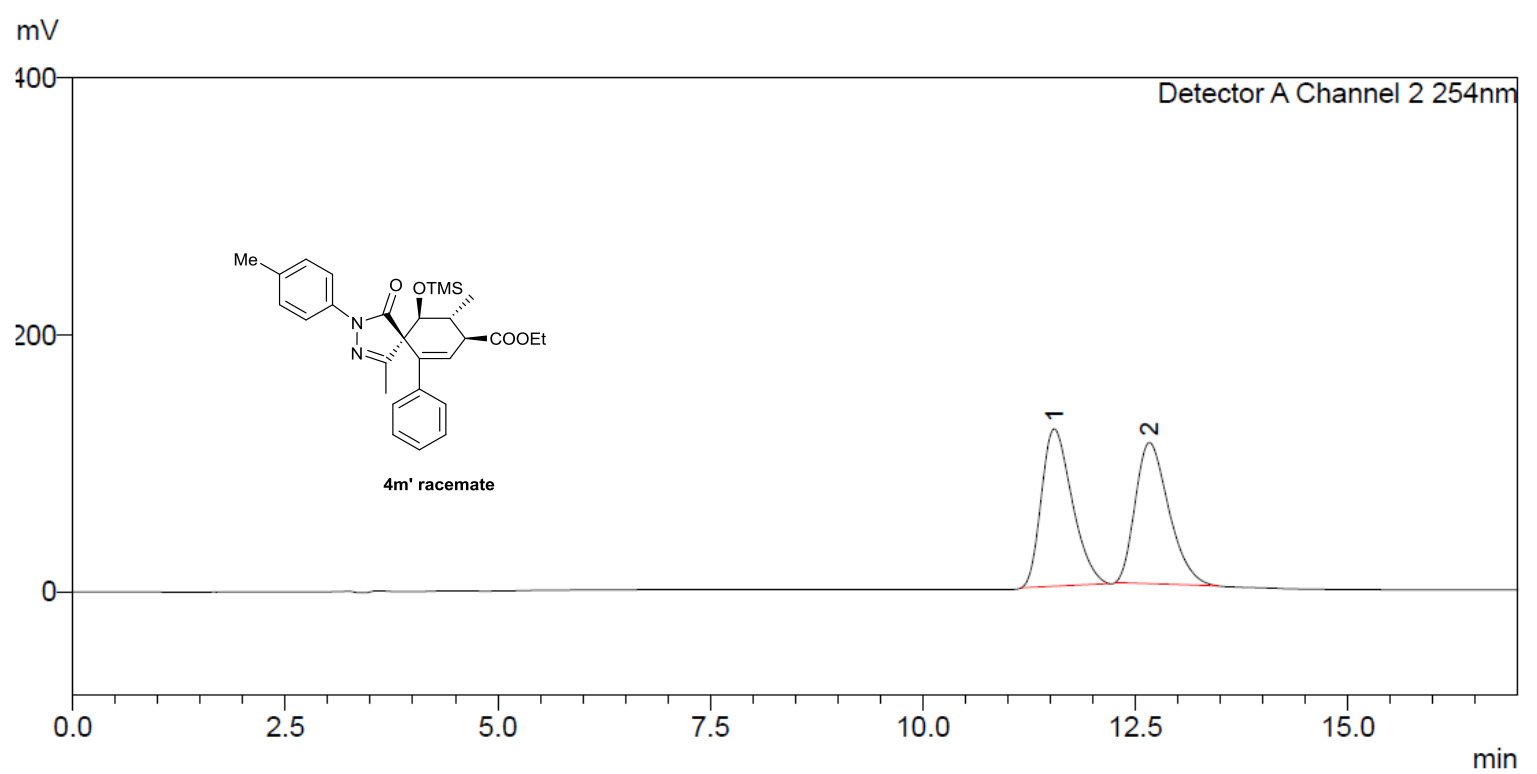

\section{Peak Analysis Report}

Detector A Channel $2254 \mathrm{~nm}$

\begin{tabular}{|c|c|c|c|c|}
\hline No. & Ret. Time & Height (mAu) & Area (mAu*min) & Rel. Area (\%) \\
\hline 1 & 11.556 & 109795 & 2784940 & 37.424 \\
\hline 2 & 12.669 & 164418 & 4656606 & 62.576 \\
\hline Total & & 274213 & 7441545 & 100.000 \\
\hline
\end{tabular}

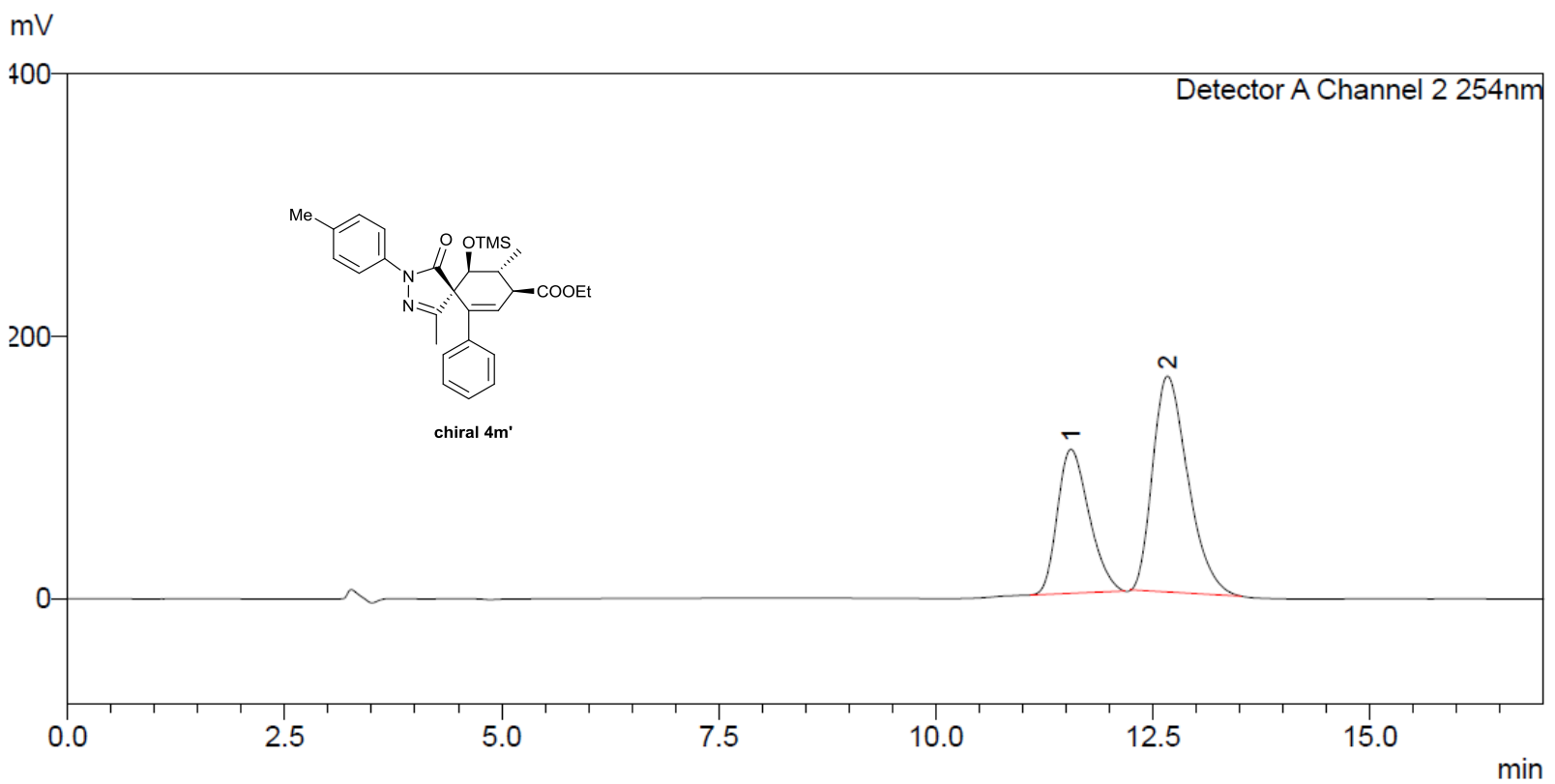



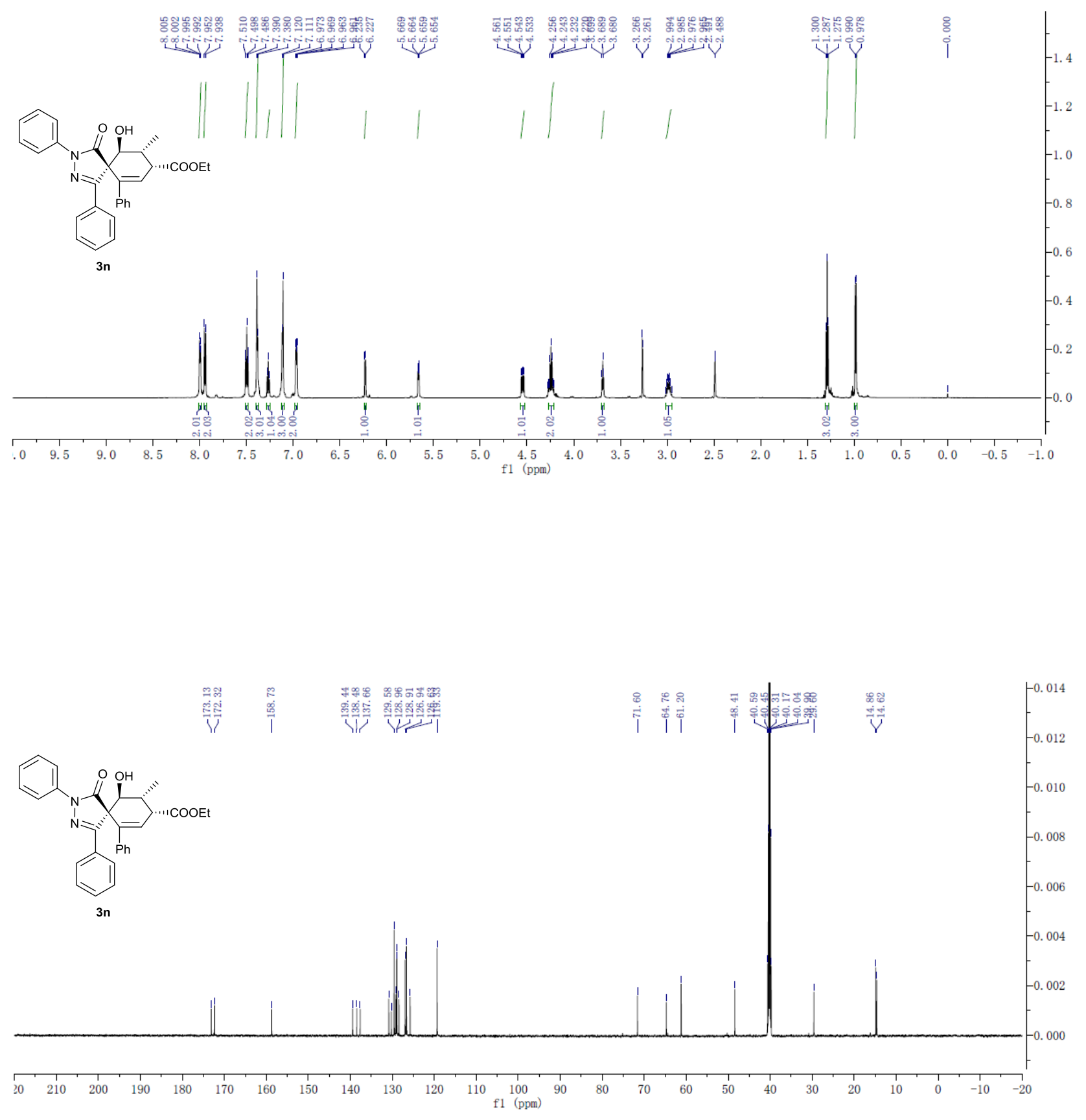

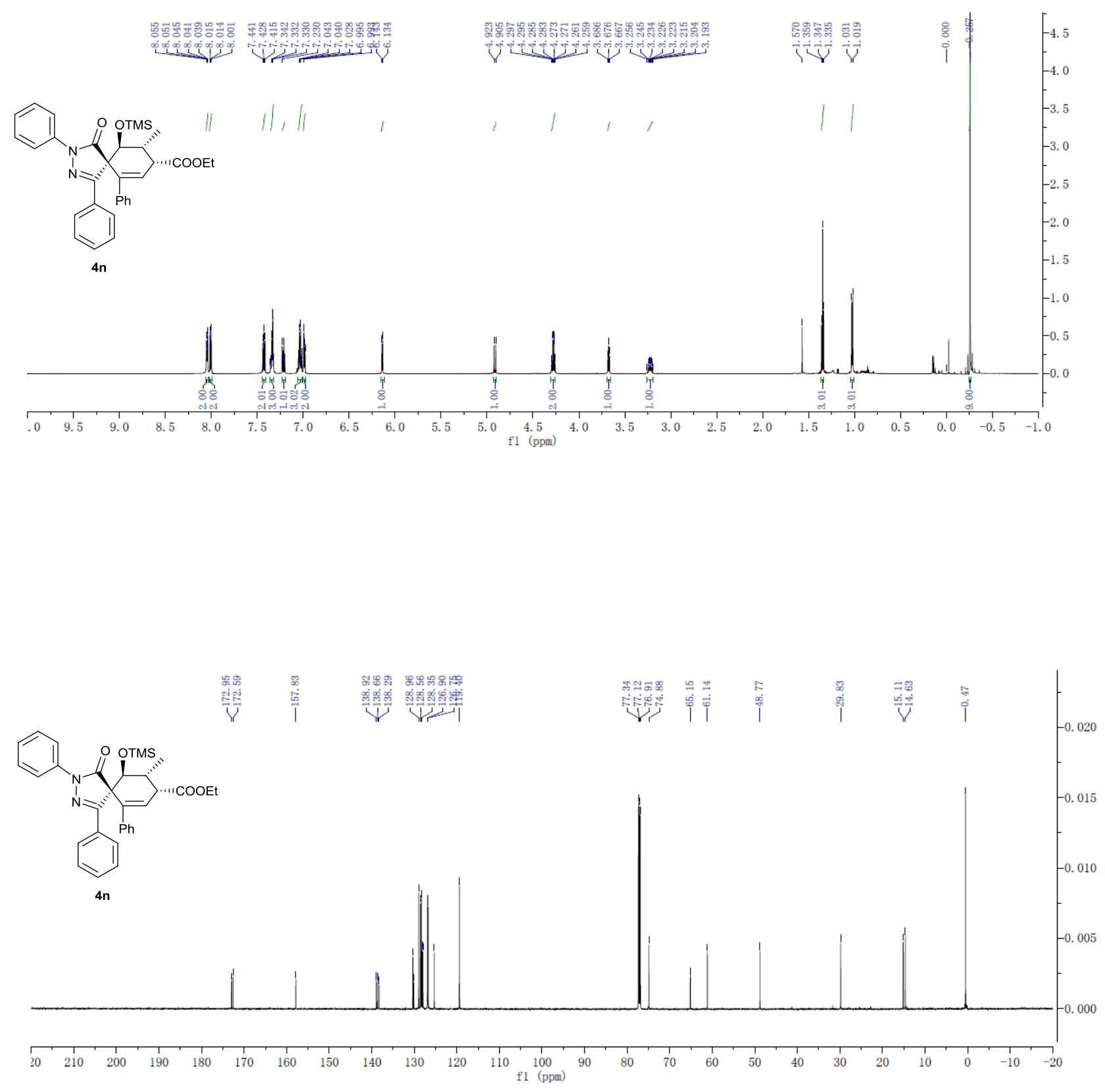


\section{Peak Analysis Report}

Detector A Channel 2 254nm

\begin{tabular}{|c|c|c|c|c|}
\hline No. & Ret. Time & Height $(\mathrm{mAu})$ & Area $\left(\mathrm{mAu}^{*} \mathrm{~min}\right)$ & Rel. Area $(\%)$ \\
\hline 1 & 10.855 & 117729 & 2131981 & 49.265 \\
\hline 2 & 29.665 & 43883 & 2195605 & 50.735 \\
\hline Total & & 161612 & 4327587 & 100.000 \\
\hline
\end{tabular}

$\mathrm{mV}$

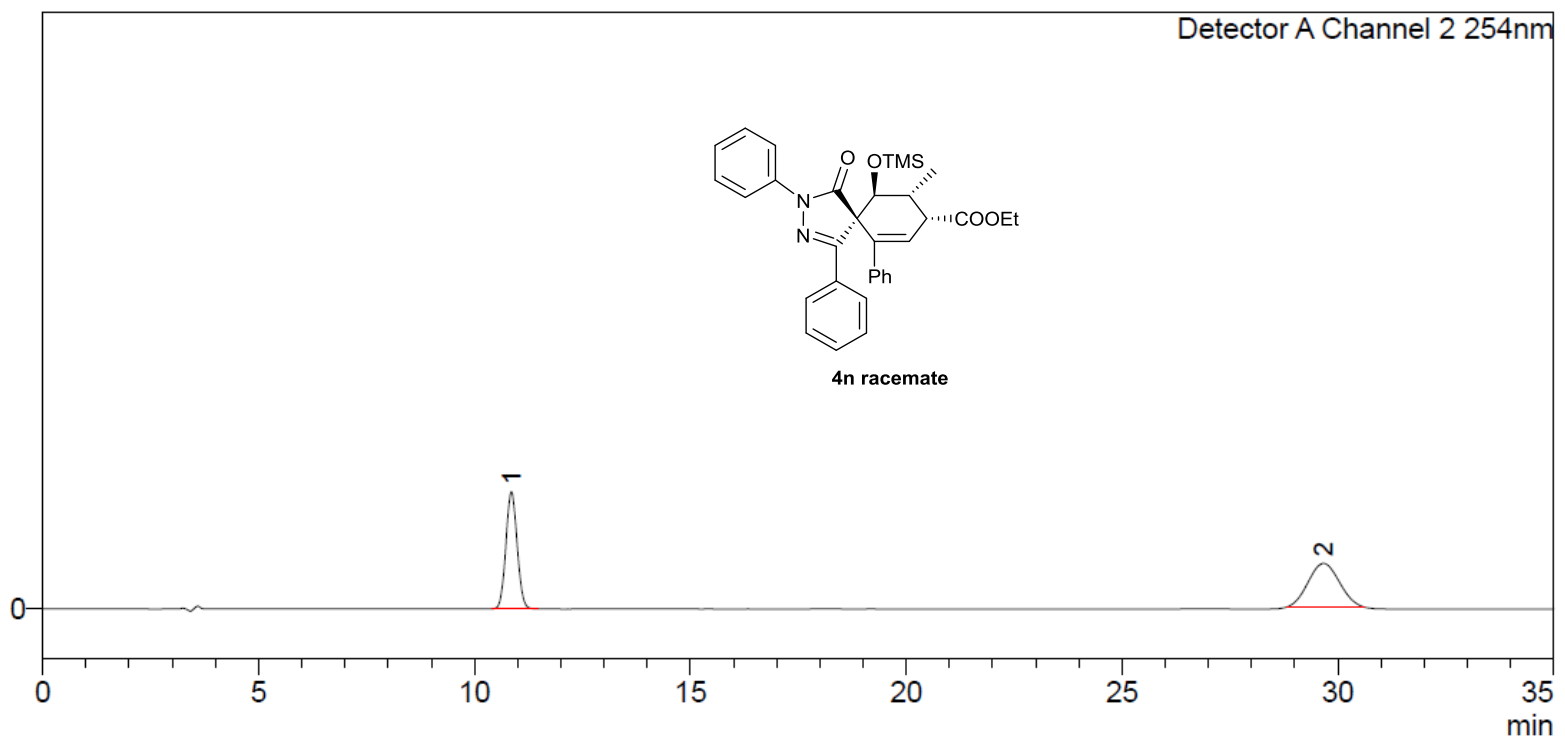

\section{Peak Analysis Report}

Detector A Channel 2 254nm

\begin{tabular}{|c|c|c|c|c|}
\hline No. & Ret. Time & Height $(\mathrm{mAu})$ & Area $\left(\mathrm{mAu}^{*} \mathrm{~min}\right)$ & Rel. Area (\%) \\
\hline 1 & 10.821 & 20953 & 372893 & 1.927 \\
\hline 2 & 29.557 & 350222 & 18976900 & 98.073 \\
\hline Total & & 371175 & 19349792 & 100.000 \\
\hline
\end{tabular}

$\mathrm{mV}$

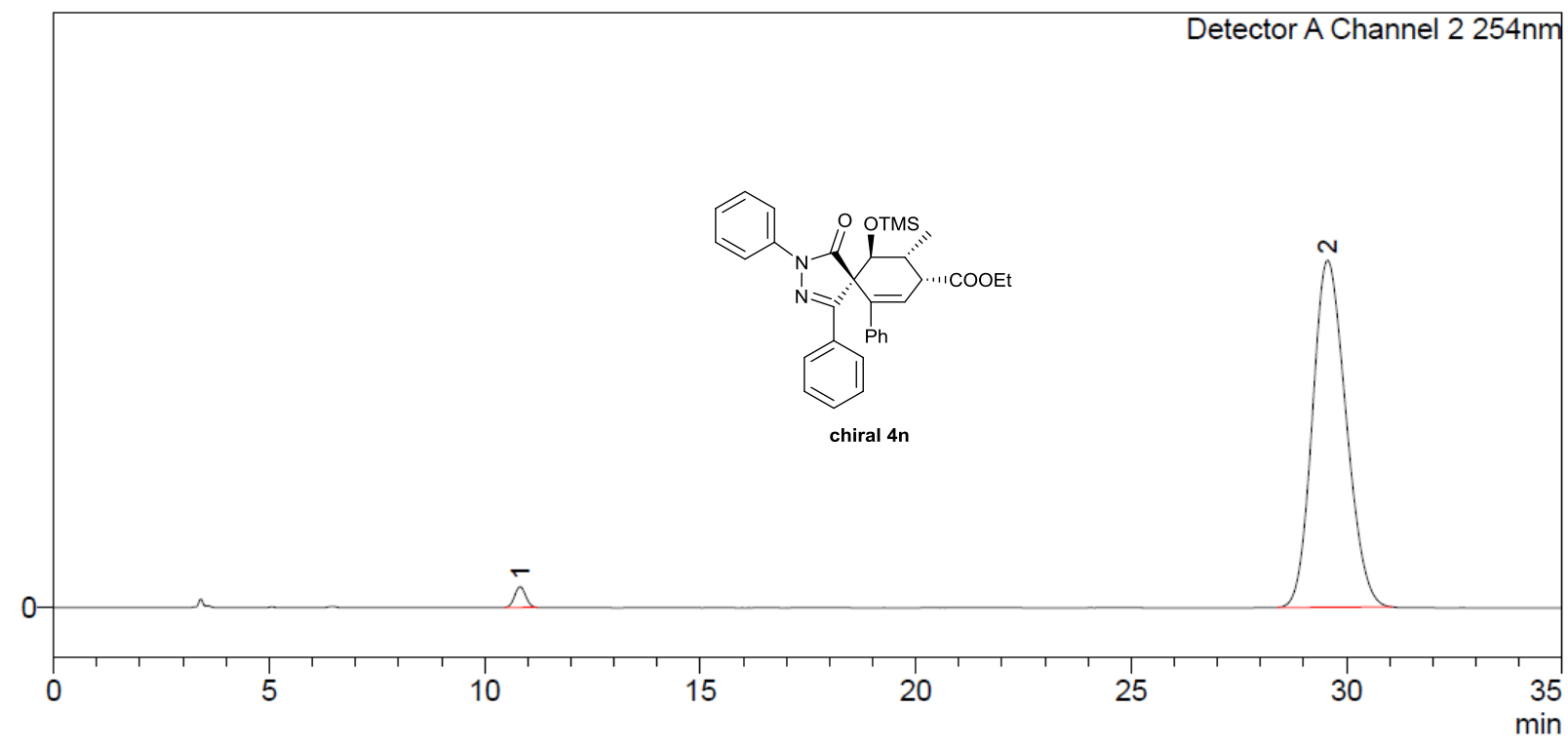



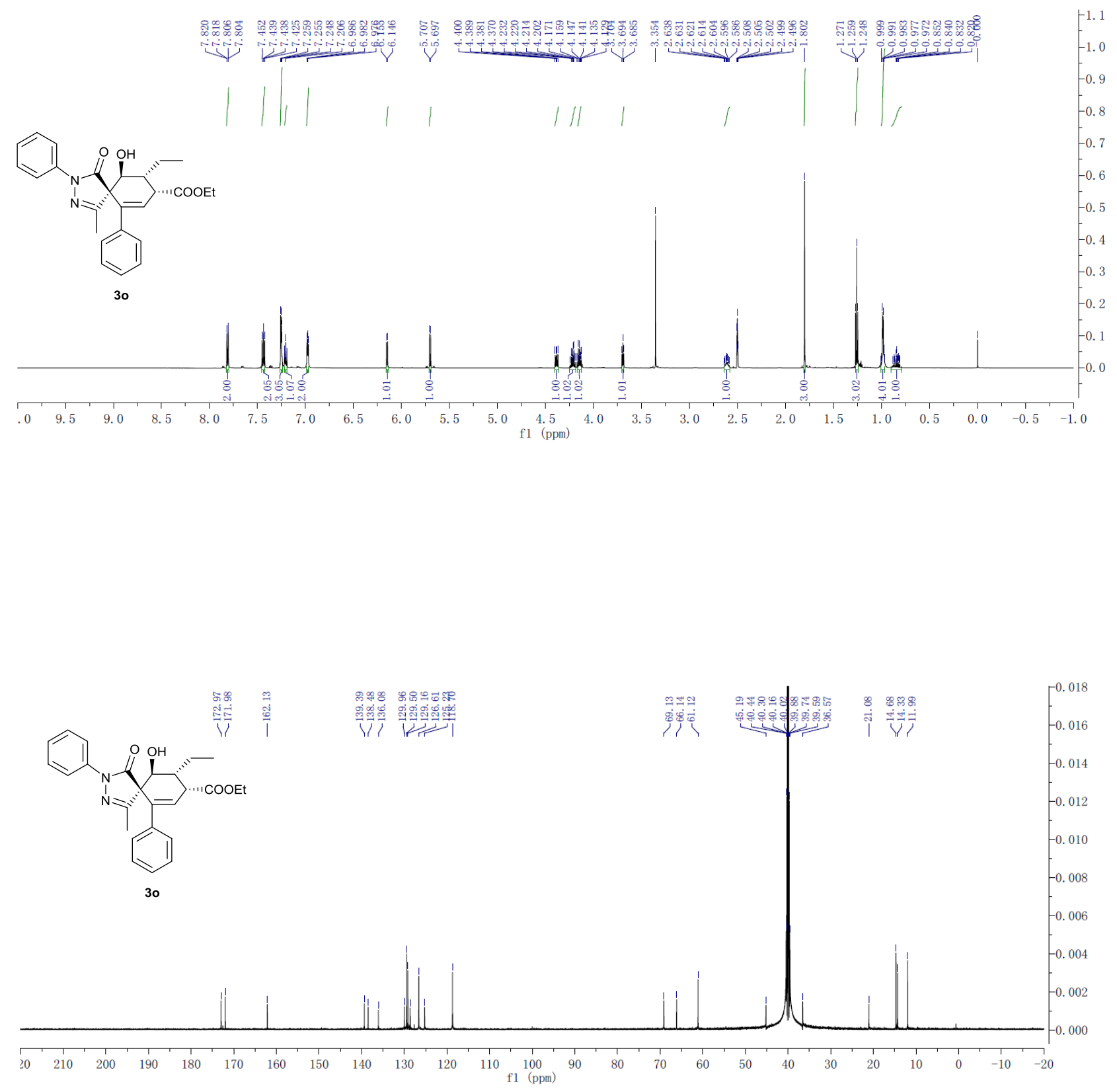


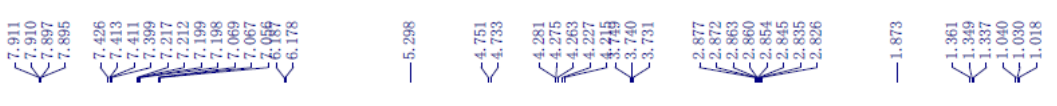

Serae

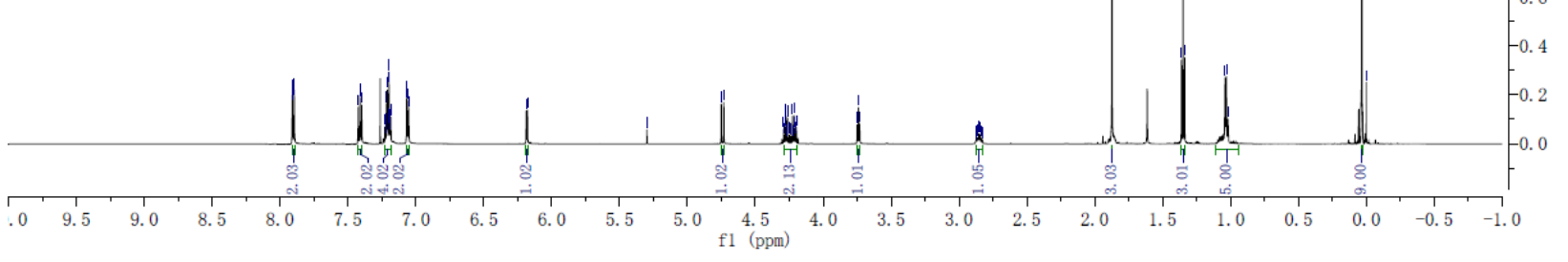

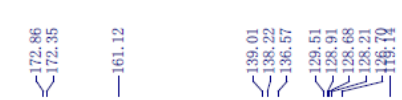

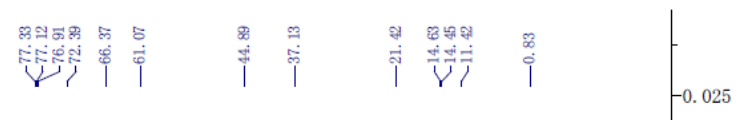

(2)

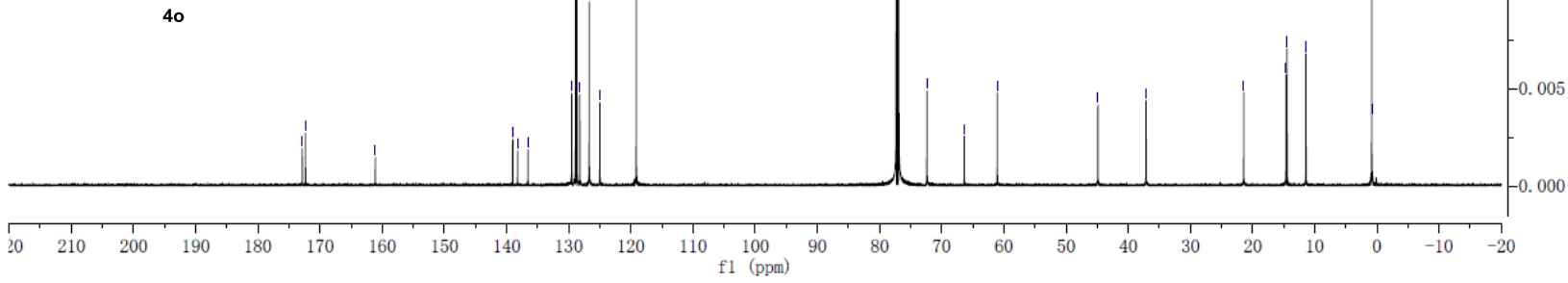




\section{Peak Analysis Report}

Detector A Channel 2 254nm

\begin{tabular}{|c|c|c|c|c|}
\hline No. & Ret. Time & Height $(\mathrm{mAu})$ & Area $\left(\mathrm{mAu}^{*} \mathrm{~min}\right)$ & Rel. Area (\%) \\
\hline 1 & 4.551 & 141622 & 1426179 & 50.089 \\
\hline 2 & 17.744 & 36281 & 1421103 & 49.911 \\
\hline Total & & 177903 & 2847283 & 100.000 \\
\hline
\end{tabular}

$\mathrm{mV}$

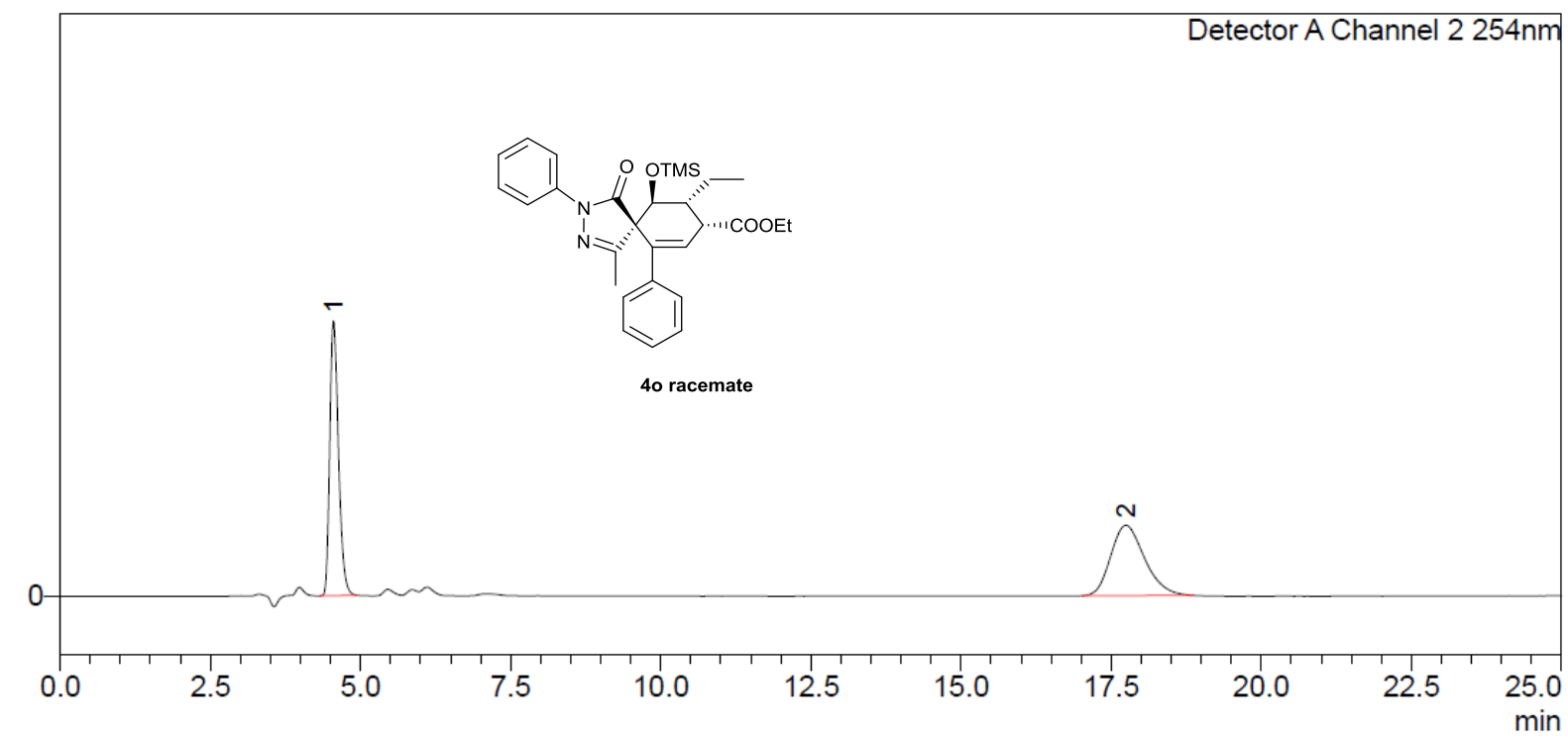

\section{Peak Analysis Report}

Detector A Channel 2 254nm

\begin{tabular}{|c|c|c|c|c|}
\hline No. & Ret. Time & Height $(\mathrm{mAu})$ & Area $\left(\mathrm{mAu}^{*} \mathrm{~min}\right)$ & Rel. Area $(\%)$ \\
\hline 1 & 4.110 & 24487 & 205447 & 2.266 \\
\hline 2 & 17.738 & 220804 & 8862887 & 97.734 \\
\hline Total & & 245292 & 9068334 & 100.000 \\
\hline
\end{tabular}

$\mathrm{mV}$

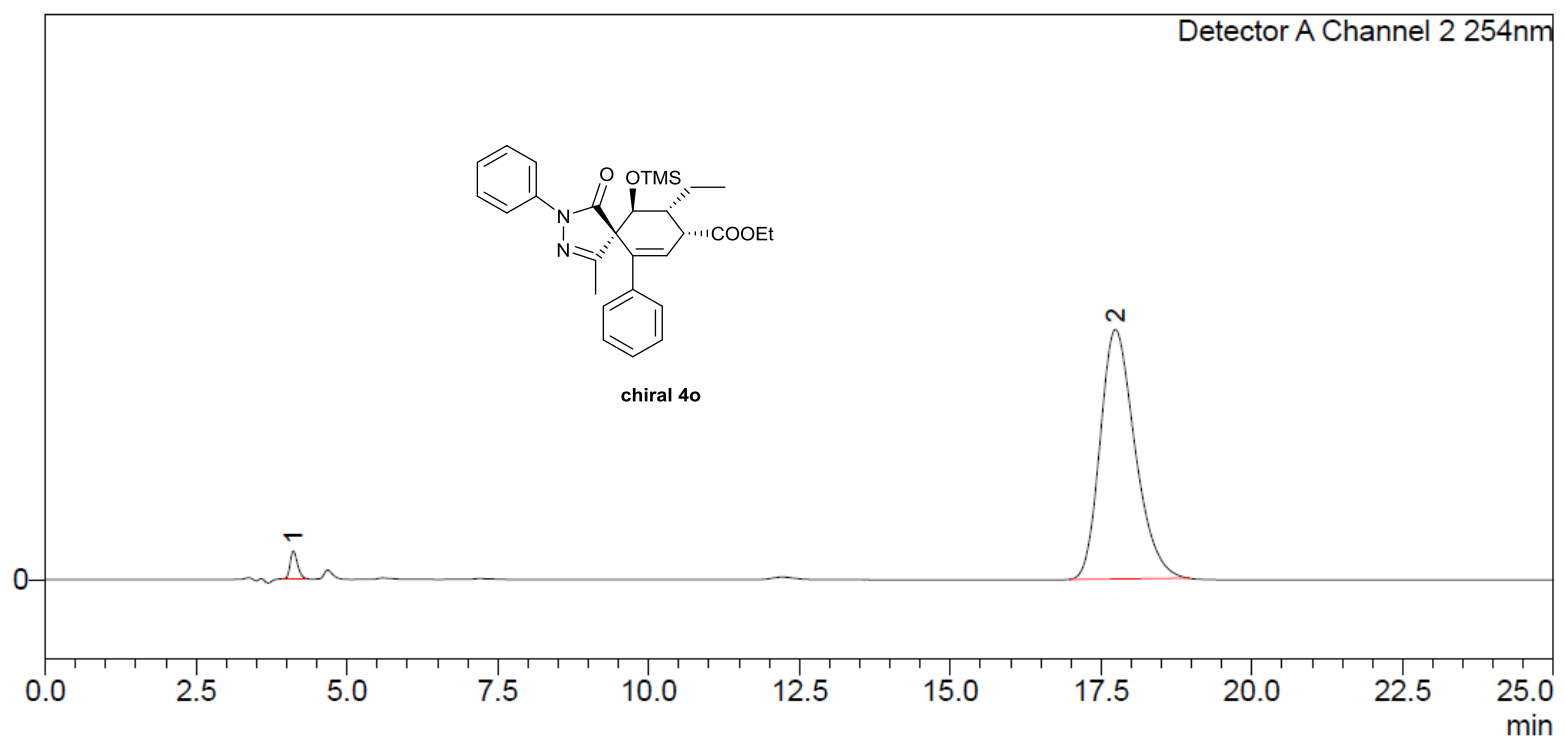



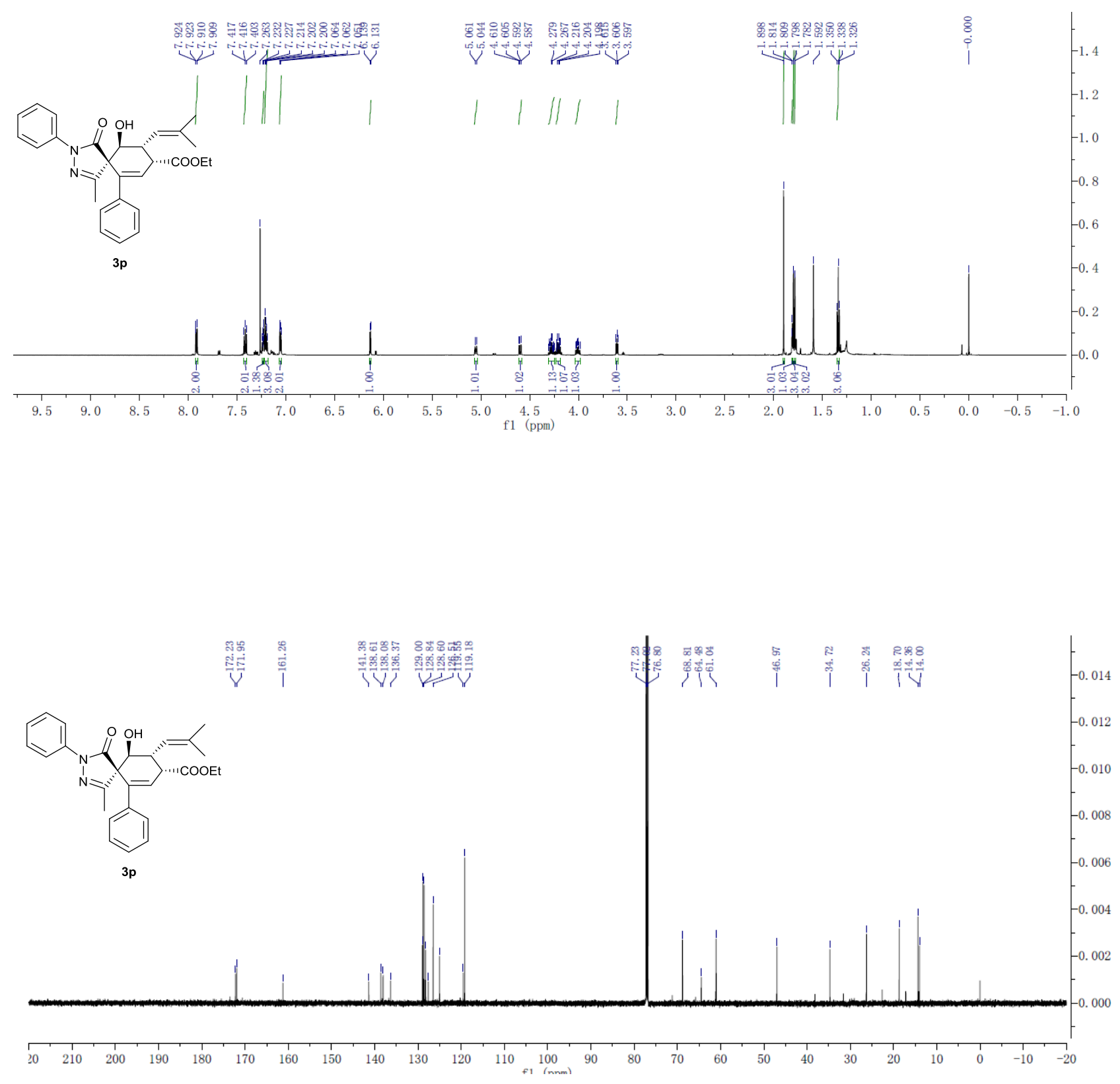

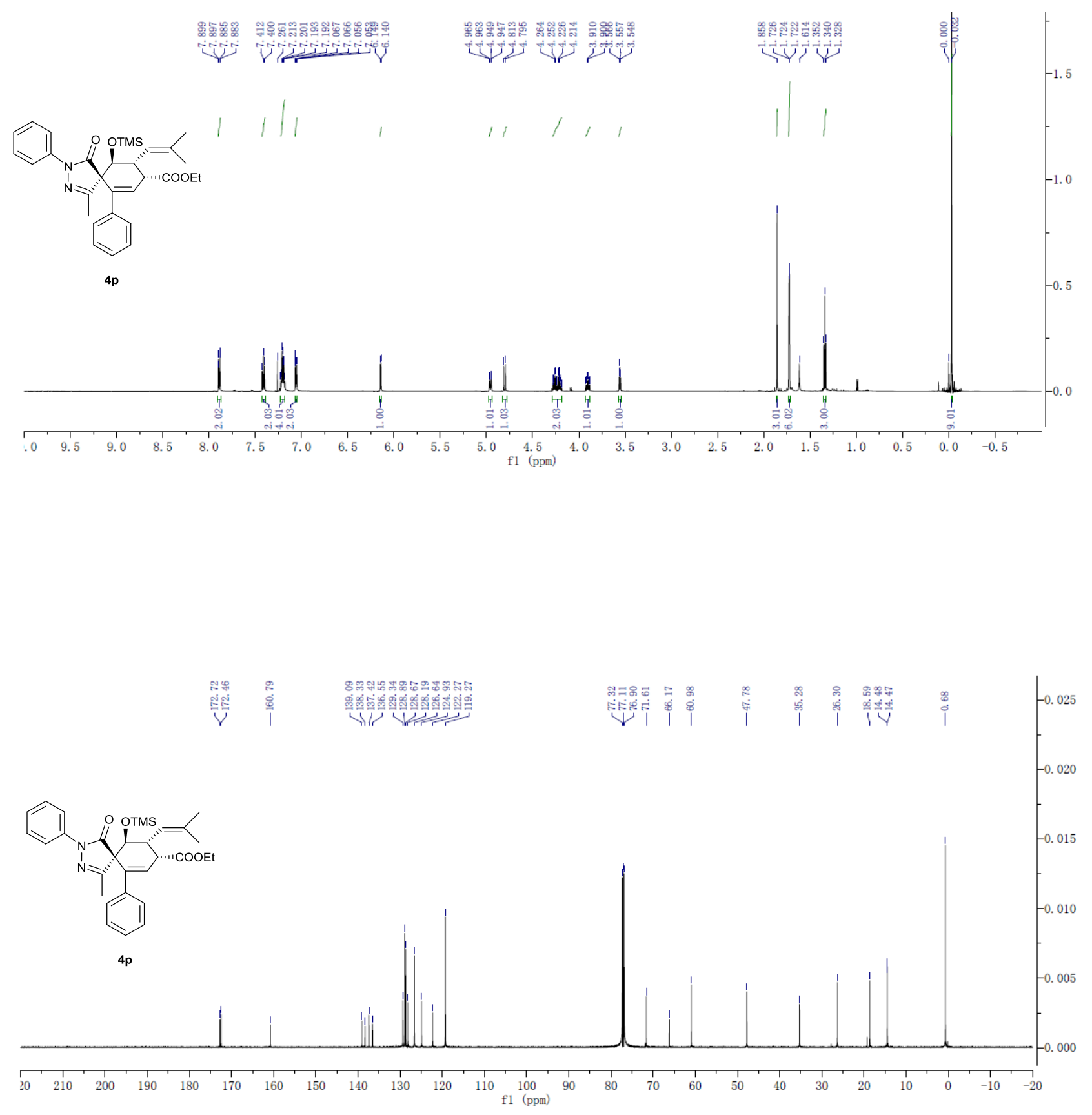
Peak Analysis Report

Detector A Channel 2 254nm

\begin{tabular}{|c|c|c|c|c|}
\hline No. & Ret. Time & Height $(\mathrm{mAu})$ & Area $\left(\mathrm{mAu}^{*} \mathrm{~min}\right)$ & Rel. Area (\%) \\
\hline 1 & 3.974 & 533358 & 4047084 & 50.532 \\
\hline 2 & 6.062 & 286359 & 3961905 & 49.468 \\
\hline Total & & 819717 & 8008989 & 100.000 \\
\hline
\end{tabular}

$\mathrm{mV}$

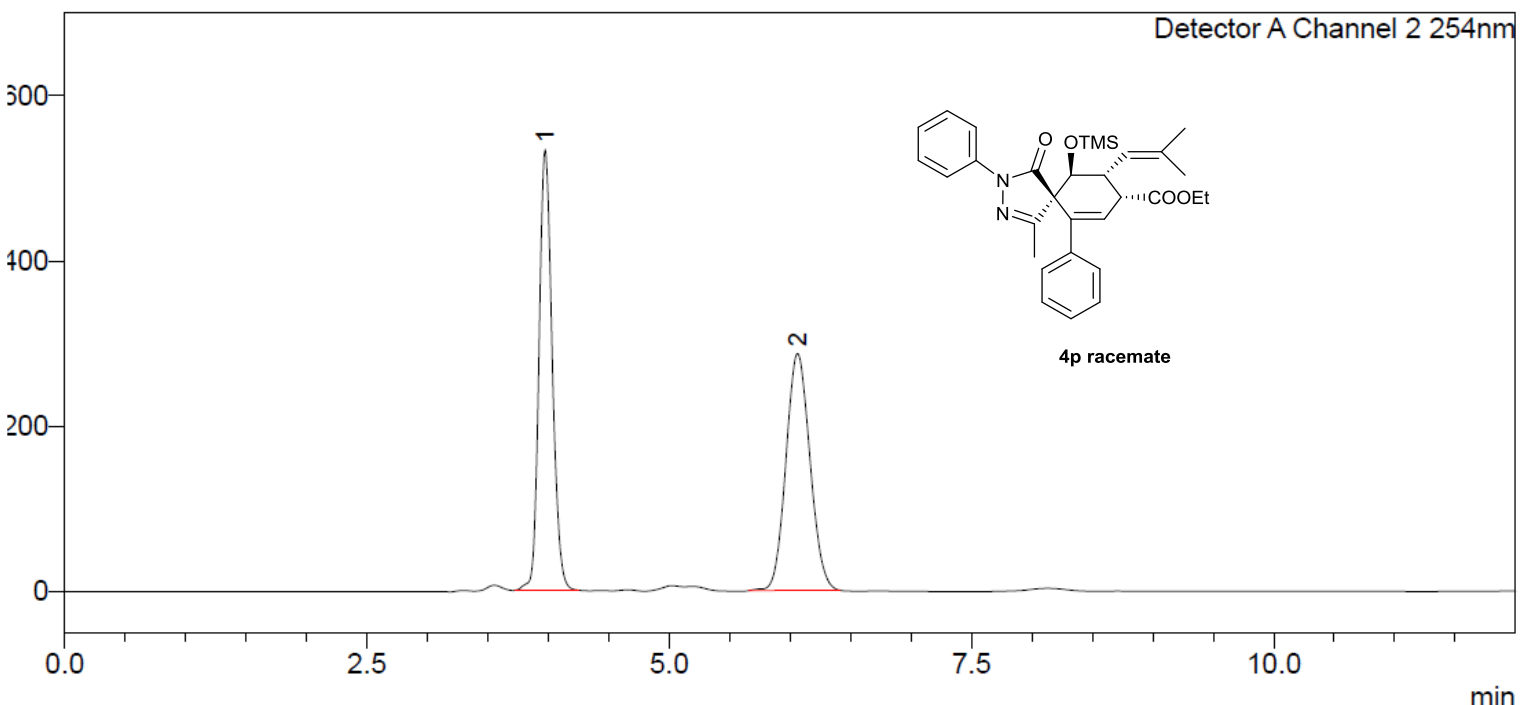

\section{Peak Analysis Report}

Detector A Channel 2 254nm

\begin{tabular}{|c|c|c|c|c|}
\hline No. & Ret. Time & Height $(\mathrm{mAu})$ & Area $\left(\mathrm{mAu}^{*} \mathrm{~min}\right)$ & Rel. Area (\%) \\
\hline 1 & 3.920 & 234880 & 1800661 & 16.918 \\
\hline 2 & 5.982 & 630427 & 8842937 & 83.082 \\
\hline Total & & 865307 & 10643598 & 100.000 \\
\hline
\end{tabular}

$\mathrm{mV}$

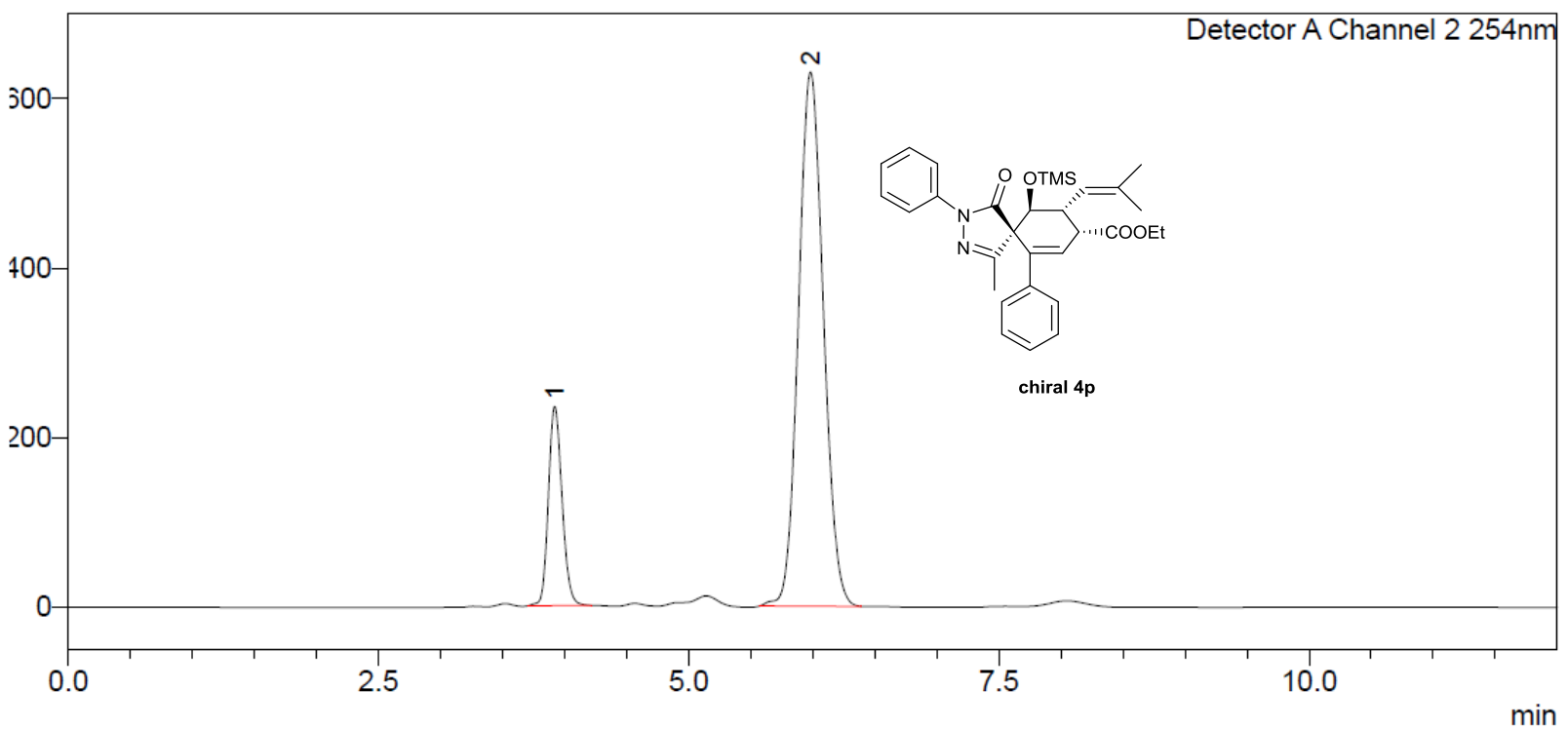



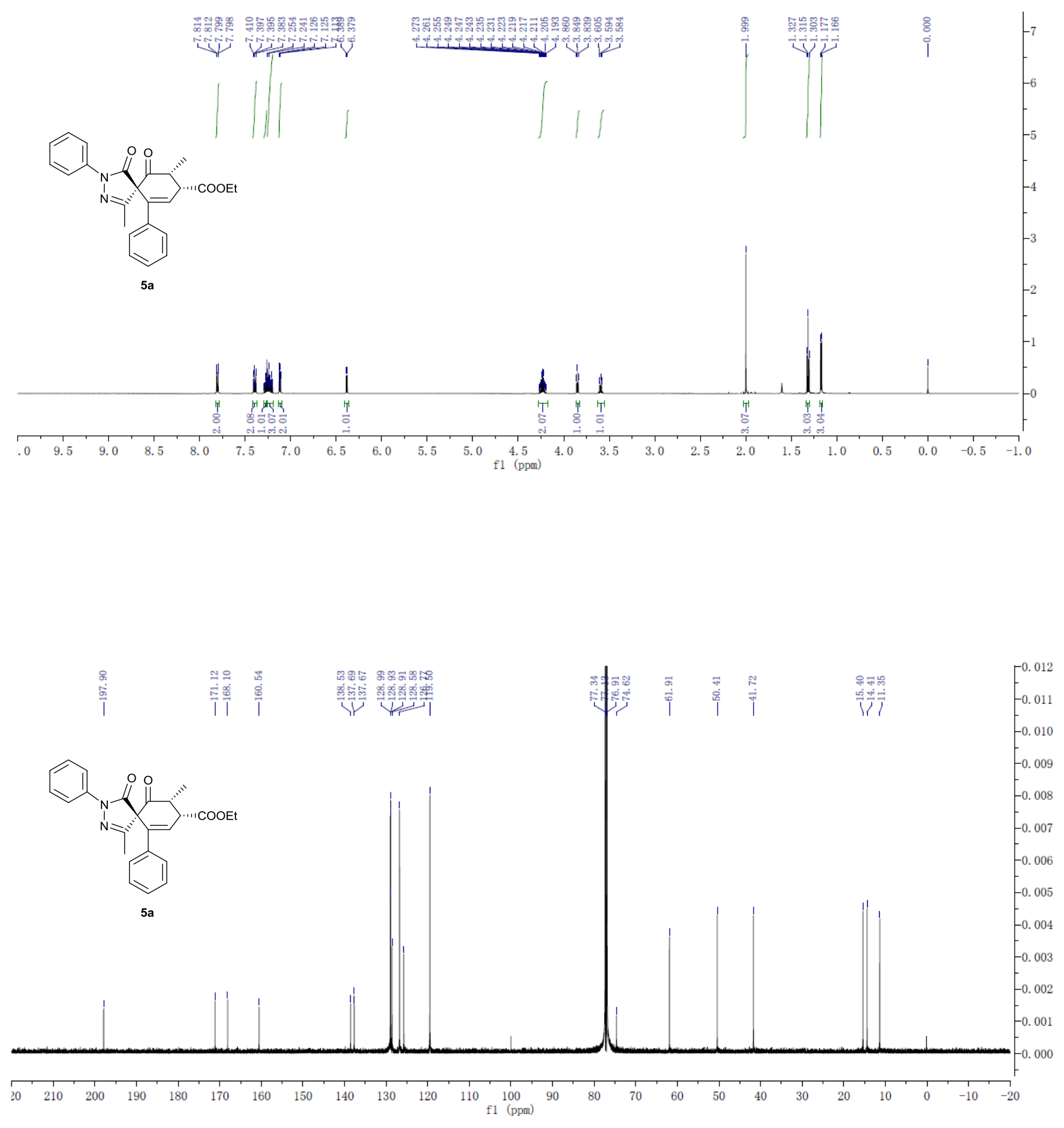


\section{Peak Analysis Report}

Detector A Channel 2 254nm
\begin{tabular}{|c|c|c|c|c|}
\hline No. & Ret. Time & Height (mAu) & Area (mAu*min) & Rel. Area $(\%)$ \\
\hline 1 & 7.497 & 297698 & 4166768 & 50.929 \\
\hline 2 & 8.760 & 234955 & 4014760 & 49.071 \\
\hline Total & & 532653 & 8181528 & 100.000 \\
\hline
\end{tabular}

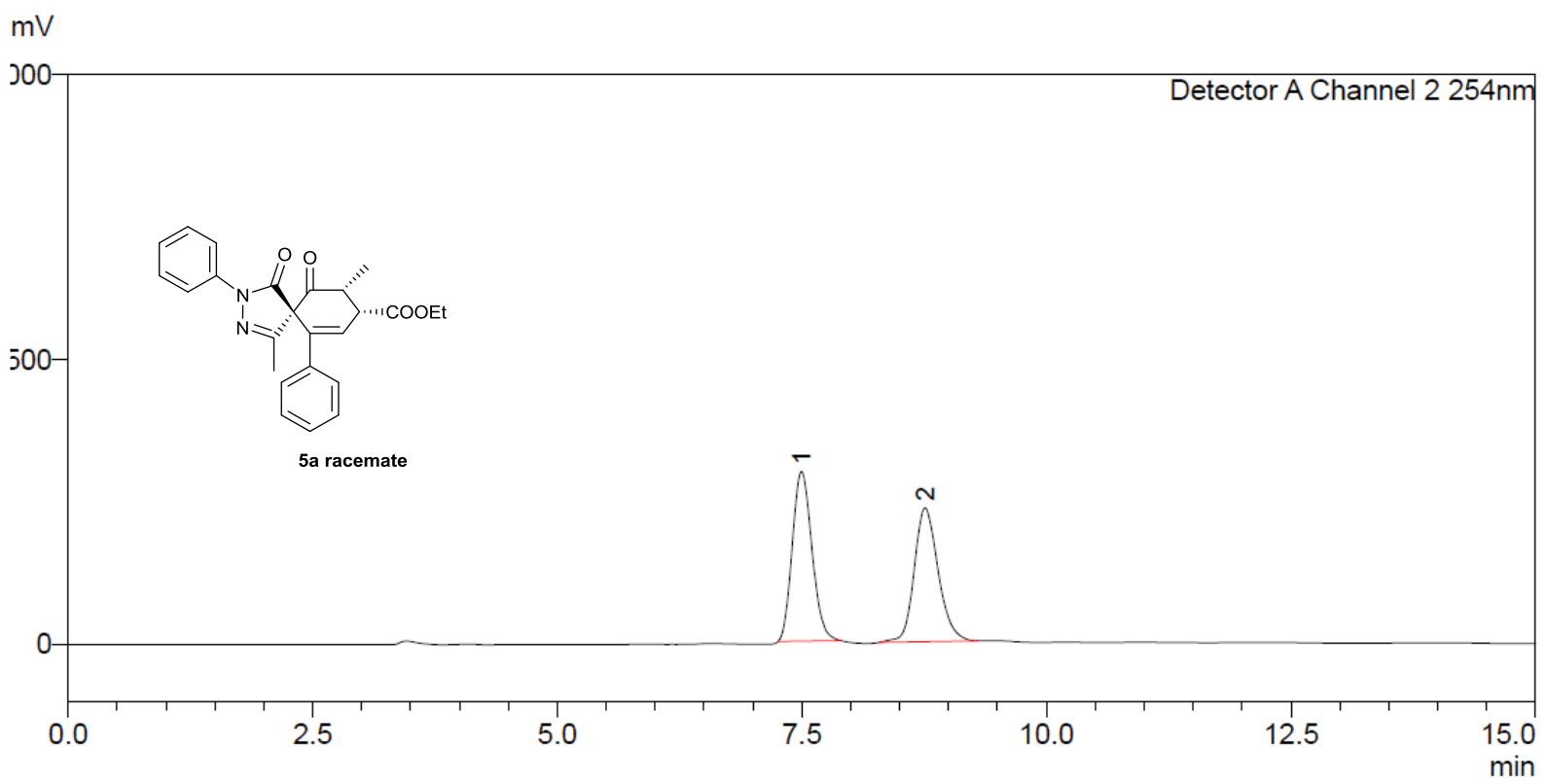

\section{Peak Analysis Report}

Detector A Channel $2254 \mathrm{~nm}$
\begin{tabular}{|c|c|c|c|c|}
\hline No. & Ret. Time & Height (mAu) & Area (mAu*min) & Rel. Area $(\%)$ \\
\hline 1 & 7.241 & 23075 & 269161 & 2.757 \\
\hline 2 & 8.444 & 574399 & 9492657 & 97.243 \\
\hline Total & & 597474 & 9761818 & 100.000 \\
\hline
\end{tabular}

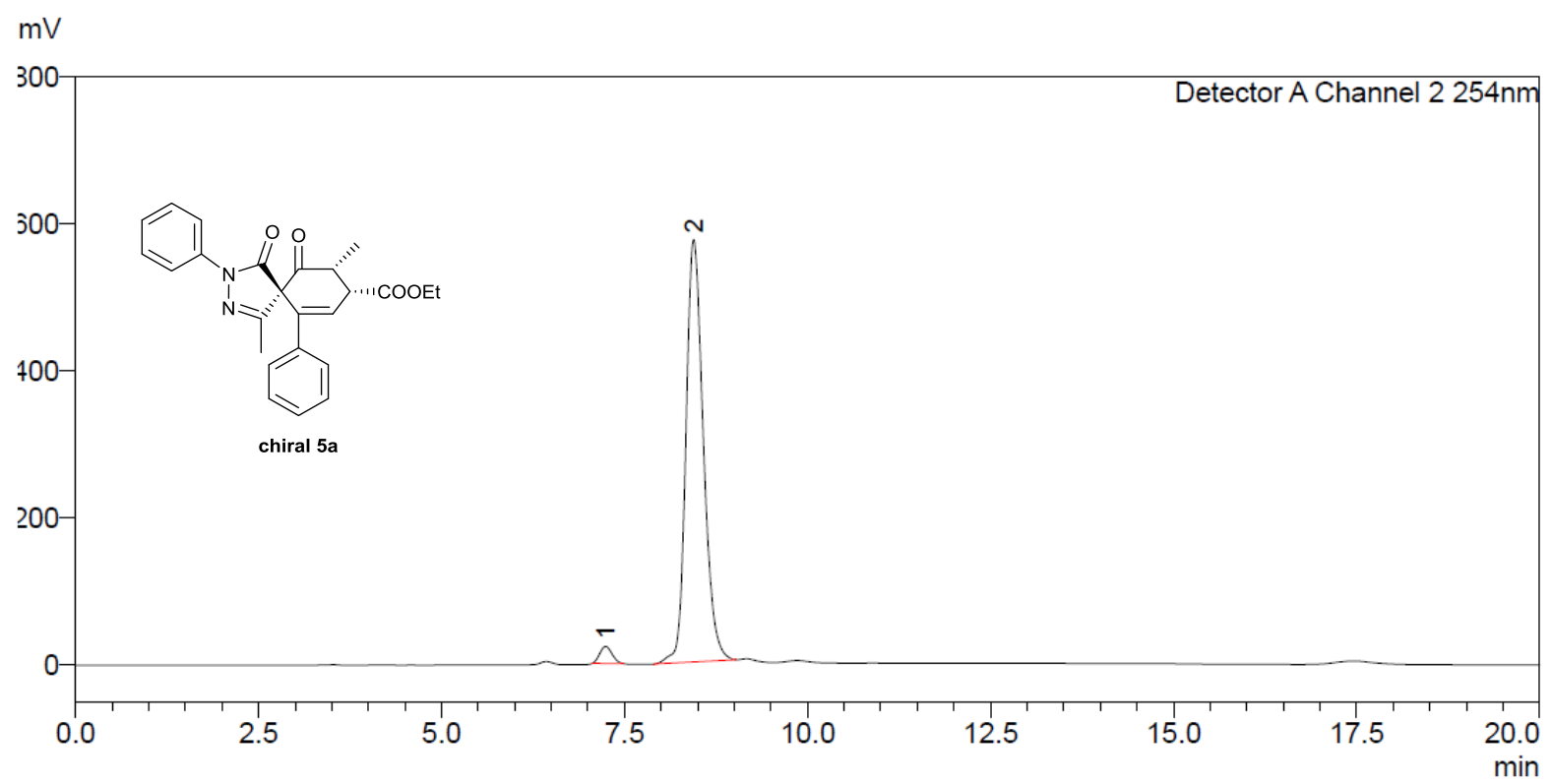




\section{The anti-proliferative IC50 values against a panel of cancer cell lines}

\section{$($ Table S1)}

\begin{tabular}{|c|c|c|c|c|c|c|}
\hline \multirow{2}{*}{ Compound } & \multicolumn{6}{|c|}{$\mathrm{IC}_{50}(\mu \mathrm{M})$} \\
\hline & BEAS-2B & A549 & MCF-7 & PANC-1 & 7860 & HCT116 \\
\hline $3 \mathbf{a}$ & 27.40 & 4.88 & 3.23 & 5.86 & 8.97 & 2.10 \\
\hline $\mathbf{3 b}$ & 12.30 & 3.02 & 2.16 & 4.65 & 6.56 & 1.66 \\
\hline $3 c$ & 14.82 & 2.99 & 2.10 & 3.57 & 4.58 & 1.57 \\
\hline 3d & 11.78 & 2.57 & 1.96 & 3.67 & 5.39 & 1.36 \\
\hline $3 \mathbf{e}$ & 15.43 & 2.97 & 2.34 & 4.58 & 5.86 & 1.53 \\
\hline $3 f$ & 39.35 & 8.73 & 5.86 & 12.56 & 18.34 & 4.80 \\
\hline $3 \mathbf{g}$ & 25.99 & 1.13 & 0.81 & 1.54 & 2.16 & 0.56 \\
\hline $3 h$ & 23.07 & 0.77 & 0.52 & 1.17 & 2.44 & 0.37 \\
\hline $3 \mathbf{i}$ & 14.79 & 3.12 & 2.17 & 3.96 & 6.26 & 1.68 \\
\hline $\mathbf{3 j}$ & 45.06 & 25.14 & 20.44 & 39.97 & 61.16 & 15.14 \\
\hline $3 \mathbf{k}$ & 42.28 & 8.29 & 6.74 & 10.53 & 14.21 & 4.68 \\
\hline 31 & 37.87 & 6.52 & 4.18 & 8.21 & 11.50 & 3.37 \\
\hline $3 m$ & 26.15 & 5.62 & 4.61 & 7.42 & 11.80 & 3.81 \\
\hline $3 n$ & 25.79 & 50.05 & 40.69 & 62.06 & $>50.00$ & 27.31 \\
\hline 30 & 26.79 & 20.11 & 14.37 & 25.14 & 35.95 & 9.15 \\
\hline $3 p$ & 41.41 & 40.78 & 27.93 & 59.54 & $>50.00$ & 17.79 \\
\hline $4 a$ & 54.50 & $>50.00$ & 46.50 & $>50.00$ & 37.80 & $>50.00$ \\
\hline $5 \mathbf{a}$ & 36.82 & 6.35 & 4.41 & 10.03 & 13.44 & 2.79 \\
\hline
\end{tabular}

${ }^{a}$ Each compound was tested in triplicate; the data are presented as the mean values. 